\begin{abstract}
BIHARI, KATHLEEN LOUISE. Analysis of Thermal Conductivity in Composite Adhesives (Under the direction of H. Thomas Banks).

Thermally conductive composite adhesives are desirable in many industrial applications, including computers, microelectronics, machinery and appliances. These composite adhesives are formed when a filler particle of high conductivity is added to a base adhesive. Typically, adhesives are poor thermal conductors. Experimentally only small improvements in the thermal properties of the composite adhesives over the base adhesives have been observed. A thorough understanding of heat transfer through a composite adhesive would aid in the design of a thermally conductive composite adhesive that has the desired thermal properties.

In this work, we study design methodologies for thermally conductive composite adhesives. We present a three dimensional model for heat transfer through a composite adhesive based on its composition and on the experimental method for measuring its thermal properties. For proof of concept, we reduce our model to a two dimensional model. We present numerical solutions to our two dimensional model based on a composite silicone and investigate the effect of the particle geometry on the heat flow through this composite. We also present homogenization theory as a tool for computing the "effective thermal conductivity" of a composite material.

We prove existence, uniqueness and continuous dependence theorems for our two dimensional model. We formulate a parameter estimation problem for the two dimensional model and present numerical results. We first estimate the thermal conductivity parameters as constants, and then use a probability based approach to estimate the parameters as realizations of random variables. A theoretical framework for the probability based approach is outlined.

Based on the results of the parameter estimation problem, we are led to formally derive sensitivity equations for our system. We investigate the sensitivity of
\end{abstract}


our composite silicone with respect to the thermal conductivity of both the base silicone polymer and the filler particles. Numerical results of this investigation are also presented. 


\title{
ANALYSIS OF THERMAL CONDUCTIVITY IN COMPOSITE ADHESIVES
}

\author{
BY \\ KATHLEEN L. BIHARI
}

\begin{abstract}
A DiSSERTATION SUBMITTED TO THE GRADUATE FACULTY OF
NORTH CAROLINA STATE UNIVERSITY

IN PARTIAL FULFILLMENT OF THE

REQUIREMENTS FOR THE DEGREE OF

DOCTOR OF PHILOSOPHY
\end{abstract}

DEPARTMENT OF MATHEMATICS

RALEIGH

August 2, 2001

APPROVED BY:

CHAIR OF ADVISORY COMMITTEE 
-- To Jon, Mom, Dad and Mikey, with all my love -- 


\section{Biography}

Kathleen L. Bihari was born on August 6, 1974 in Baltimore, Maryland to Lewis and

Louise Gaston. She graduated summa cum laude from Western Maryland College in 1996 with a B.A. in Mathematics. In 1998 she earned an M.S. in Mathematics from the University of Virginia. She married Jonathan Bihari on June 20, 1999. 


\section{Acknowledgments}

First, I would like to thank my advisor Dr. H. T. Banks. His knowledge and enthusiasm were an essential ingredient in this dissertation. I am particularly grateful that he was willing to take me on as a student, despite his numerous other responsibilities. I would also like to thank the members of my committee, Dr. Tran, Dr. Ito and Dr. Fouque, for their valuable contributions.

I would like to thank Lord Corporation for their endowment of the Lord Corporation Fellowship of which I was honored to be the first recipient. There are many people at Lord Corporation whose time and effort are greatly appreciated. I would especially like to thank Jim Hogan for tirelessly answering my questions and letting me help out with experiments in the lab. A huge thanks is also due Robin Tirpak for his commitment to this problem. In addition, I would like to thank Robert Kyles, Robert Williams, Scott Durso and Mark Pressley for their contributions.

I am grateful to Lisa Stanley for sharing her knowledge of sensitivity equations and, in particular, her preprints on the subject with me.

Mom, Dad and my "little" brother Mike, thank you for your unconditional love and for always supporting and encouraging my goals. I know I wouldn't be where I am today without you!

Finally, to my husband and best friend, Jon, go my unending love and thanks. I couldn't have made it through this without your love, support, encouragement and ability to make me laugh when I needed it most! The past few years with you have been wonderful and I know the future will be even better. 


\section{Table of Contents}

List of Figures $\quad$ vii

List of Tables $\quad$ ix

1 Introduction 1

2 Experimental Method $\mathbf{5}$

2.1 Composition of the Composite Silicone . . . . . . . . . . . . 5

2.2 Data Collection . . . . . . . . . . . . . . . 6

2.3 Sample Data. . . . . . . . . . . . . . . . . . . . 9

3 A Model for Heat Transfer through a Composite Silicone 14

3.1 Three Dimensional Model . . . . . . . . . . . . . . . . . . . . 14

3.2 Two Dimensional Model . . . . . . . . . . . . . . . . . . . . 17

3.3 Matlab PDE Toolbox Solutions . . . . . . . . . . . . . . . . . 19

3.4 Different Particle Geometries . . . . . . . . . . . . . . . 25

4 Homogenization $\quad 36$

4.1 Model with Periodic Microstructure . . . . . . . . . . . . . . 36

4.2 Homogenization in $n$-Dimensions . . . . . . . . . . . . . . . . . . . . . . . . . . . . . . .

4.3 Homogenization in Two Dimensions . . . . . . . . . . . . . . . 43

4.4 Using Matlab to Find the Homogenized Coefficient . . . . . . . . . . 45

5 Well-Posedness Results 48

5.1 Problem in Sesquilinear Form . . . . . . . . . . . . . . . . . 48

5.1.1 Preliminaries . . . . . . . . . . . . . . . . . 48

5.1 .2 Weak Solution . . . . . . . . . . . . . . . 51

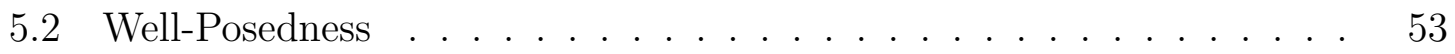

5.2.1 Existence and Uniqueness . . . . . . . . . . . . . . . 53

5.2.2 Continuous Dependence on Initial Data and Forcing Function 62 
5.3 Continuous Dependence on Parameters . . . . . . . . . . . . . 63

5.3.1 Continuous Dependence on $k$ and $h \ldots \ldots \ldots . \ldots . \ldots . \ldots 63$

5.3.2 Continuous Dependence on $\rho$ and $c_{p} \ldots \ldots \ldots 6$

6 Parameter Estimation for Two Dimensional Heat Transfer Problem 71

6.1 Preliminaries . . . . . . . . . . . . . . . . . . . . 71

6.2 Estimating Constant Parameters . . . . . . . . . . . . . . 73

6.3 Estimating Parameters as Random Variable . . . . . . . . . . 76

6.3.1 Parameter Estimation in Nonlinear Systems . . . . . . . . . . 77

6.3.2 The Prohorov Metric in the Space of Probability Distributions 79

6.3.3 Stability of the Inverse Problem . . . . . . . . . . . . . 84

6.4 Estimating the Thermal Conductivity Parameters as Random Variables 87

7 Sensitivity Equations $\quad 91$

7.1 Formal Derivation using Fréchet Derivatives . . . . . . . . . . . 91

7.2 Sensitivity to the Particle Thermal Conductivity . . . . . . . . . 98

7.2.1 Derivation of Sensitivity Equations . . . . . . . . . . . . 98

7.2.2 Weak Solution to Sensitivity Equation . . . . . . . . . 100

7.2 .3 Well-Posedness . . . . . . . . . . . . . . . . . . . 103

7.2 .4 Matlab Solutions . . . . . . . . . . . . . . . . . . . 104

7.3 Sensitivity to the Silicone Polymer Thermal Conductivity . . . . . . . 110

7.3.1 Derivation of Sensitivity Equations . . . . . . . . . . 110

7.3.2 Matlab Solutions . . . . . . . . . . . . . . . . . . 111

8 Conclusions and Future Work $\quad 117$

8.1 Conclusions . . . . . . . . . . . . . . . . . . . . . . . . . . 117

8.2 Future Work . . . . . . . . . . . . . . . . . . . . . . . 118

$\begin{array}{ll}\text { List of References } & 120\end{array}$ 


\section{List of Figures}

2.1 Schematic diagram of Holometrix Microflash . . . . . . . . . . . . 7

3.1 Three dimensional heat transfer model . . . . . . . . . . . . . . . 15

3.2 Three dimensional composite silicone boundaries . . . . . . . . . . . . 16

3.3 Two dimensional heat transfer model . . . . . . . . . . . . . . . . 18

3.4 Two dimensional composite silicone slice . . . . . . . . . . . . . . . . 18

3.5 Two dimensional composite silicone geometry . . . . . . . . . . . 20

3.6 PDE Toolbox solution at $t_{1}, t_{2}, \ldots, t_{8} \ldots \ldots . \ldots 24$

3.7 Close up segment of solution at $t_{2} \ldots \ldots \ldots \ldots$. . . . . . . . 25

3.8 Temperature at $\gamma_{2}$ boundary at time $t_{2} \ldots \ldots \ldots . \ldots 25$

3.9 Two dimensional composite slice . . . . . . . . . . . . . . 26

3.10 Uniform geometry, 8 particles of radius 22.5 . . . . . . . . . . . . . 29

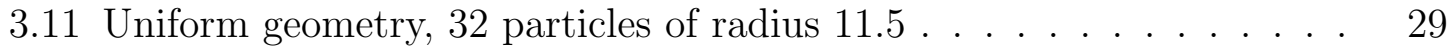

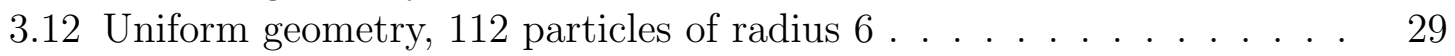

3.13 Uniform geometry, 20 particles particles of radius 22.5 and 11.5 satisfying a 1:4 distribution . . . . . . . . . . . . . . . . . . . . . . 29

3.14 Uniform geometry, 49 particles particles of radius 22.5, 11.5, and 0.625 satisfying a 4:16:39 distribution . . . . . . . . . . . . . . . . . 29

3.15 Shifted geometry, 31 particles of radius $11.5 \ldots 30$

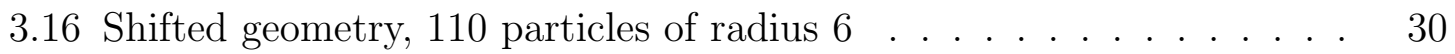

3.17 Random geometry, 8 particles particles of radius 22.5 . . . . . . . . 30

3.18 Random geometry, 8 particles particles of radius 22.5 . . . . . . . . . 30

3.19 Random geometry, 31 particles particles of radius 11.5 . . . . . . . . 30

3.20 Random geometry, 31 particles particles of radius 11.5 . . . . . . . . 31

3.21 Random geometry, 44 particles particles of radius $22.5,11.5$, and 0.625 satisfying a 1:3:7 distribution . . . . . . . . . . . . . . 31

3.22 Random geometry, 44 particles particles of radius $22.5,11.5$, and 0.625 satisfying a 1:3:7 distribution . . . . . . . . . . . . . 31

3.23 Random geometry, 44 particles particles of radius $22.5,11.5$, and 0.625 satisfying a 1:3:7 distribution . . . . . . . . . . . . . . . . 31 
3.24 Solution (left) and flux at heat sink interface (right) using the uniform geometry with same sized particles in Figure $3.11 \ldots . . . . . .32$

3.25 Solution (left) and flux at heat sink interface (right) using the shifted geometry with same sized particles in Figure 3.15 . . . . . . . . 32

3.26 Solution (left) and flux at heat sink interface (right) using the random geometry with same sized particles in Figure 3.19 . . . . . . . . .

3.27 Solution (left) and flux at heat sink interface (right) using the random geometry with three different sized particles in Figure $3.21 \ldots 33$

4.1 An example of a periodic microstructure in 2 dimensions $\ldots . . . .37$

4.2 Periodicity cell,$Y \ldots \ldots \ldots \ldots \ldots \ldots \ldots$

6.1 Cost $J_{p}\left(\bar{q}_{p}\right)$ for $\bar{q}_{p}=10,11,12, \ldots, 800 \ldots \ldots \ldots \ldots \ldots \ldots$

6.2 Cost $J_{s}\left(\bar{q}_{s}\right)$ for $\bar{q}_{s}=0,0.001,0.002, \ldots, 1 \ldots \ldots \ldots \ldots$

7.1 Average sensitivity at $\gamma_{2}$ at times $t_{2}, t_{3}, t_{4}$, and $t_{5}$ as a function of $q_{p} \quad 106$

7.2 Average sensitivity at $\gamma_{2}$ at times $t_{6}, t_{7}$, and $t_{8}$ as a function of $q_{p} \ldots 107$

7.3 Relative average sensitivity at $\gamma_{2}$ at times $t_{2}, t_{3}, t_{4}$, and $t_{5}$ as a function of $q_{p} \ldots \ldots \ldots \ldots \ldots \ldots \ldots \ldots$

7.4 Relative average sensitivity at $\gamma_{2}$ at times $t_{6}, t_{7}$, and $t_{8}$ as a function of $q_{p} \ldots \ldots \ldots \ldots \ldots \ldots \ldots \ldots$

7.5 Average sensitivity at $\gamma_{2}$ at times $t_{2}, t_{3}, t_{4}$, and $t_{5}$ as a function of $q_{s} \quad 113$

7.6 Average sensitivity at $\gamma_{2}$ at times $t_{6}, t_{7}$, and $t_{8}$ as a function of $q_{s} \ldots 114$

7.7 Relative average sensitivity at $\gamma_{2}$ at times $t_{2}, t_{3}, t_{4}$, and $t_{5}$ as a function

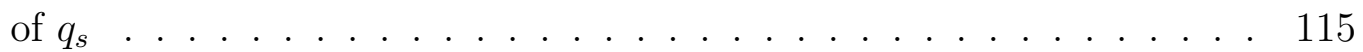

7.8 Relative average sensitivity at $\gamma_{2}$ at times $t_{6}, t_{7}$, and $t_{8}$ as a function

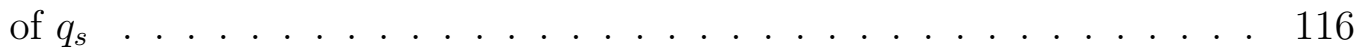




\section{List of Tables}

2.1 Data from test piece 25-1, 25\% by volume Grade 6 aluminum particles 10

2.2 Temperature rise for three test pieces using equations (2.3) and (2.5) 11

2.3 Data from $25 \%$ by volume test pieces . . . . . . . . . . . . . . . 12

2.4 Data from $35 \%$ by volume test pieces . . . . . . . . . . . . . . . 12

2.5 Data from $45 \%$ by volume test pieces . . . . . . . . . . . 13

3.1 Model parameters . . . . . . . . . . . . . . . . . . . . . . . 21

3.2 Times in milliseconds . . . . . . . . . . . . . . . . . . . . 22

3.3 Measurements of the flux at the interface between composite and heat sink for different geometries . . . . . . . . . . . . . . . . . 35

6.1 Optimization results for estimating a constant value for $k_{p} \ldots \ldots$. . . 73

6.2 Optimization results for estimating a constant value for $k_{s} \ldots \ldots$. . . 74

6.3 Estimating distribution of $k_{p} \ldots \ldots \ldots \ldots$. . . . . . . . . . . . . . . . . . . . . . 89

6.4 Estimating distribution of $k_{s} \ldots \ldots \ldots$. . . . . . . . . 90 


\section{Chapter 1}

\section{Introduction}

Adhesives such as epoxies, gels, and greases have numerous commercial and industrial applications. They are found in computers, machinery, home appliances, etc. In general, these adhesives are very poor conductors. In many applications it would be advantageous to increase their thermal conductivity. For example, consider an epoxy which is used to attach a computer chip to a motherboard. During use, the flow of electricity will cause the chip to heat up. Ideally, one would like to prevent the heat from building up and potentially damaging the chip. As a solution, researchers have been studying thermally conductive composites or filled materials: base materials such as epoxies, gels, and greases, which are filled with thermally conductive particles. Filler particles, such as diamond dust, carbon fibers, or aluminum particles, with higher thermal conductivities are added to create a composite material that is a better thermal conductor than the original material. These thermally conductive composites could then replace the poorly conducting adhesives currently in use in applications like microelectronics, circuit boards, heat exchangers, machinery, and appliances.

Adding particles with a high thermal conductivity has not had as significant an impact on the overall or effective thermal conductivity of the composite as anticipated. In order to address this issue, we study design methodologies for these composite materials. Our goal is an improved understanding of heat transfer through a composite 
material and an increased knowledge of the impact the composite design has on this process.

The goal in creating a thermally conductive composite is a significant increase in the thermal conductivity of the composite over the thermal conductivity of the base material. There are several design considerations. First, the composite material should retain the desirable properties of the base material. For example, suppose our goal is a thermally conductive composite epoxy. We would want the composite epoxy to retain the features of the base epoxy, such as its ability to form a strong bond. Keeping this requirement in mind, the next consideration is the choice of filler particle. We would not want to choose a filler particle that counteracts the desirable properties of the base material. The filler particles employed to date tend to have a much higher thermal conductivity than the base material, in hopes that the composite material will have a significantly higher thermal conductivity than the base material. However, the large difference between the thermal conductivity of the base material and the filler particles may impact the heat flow through the composite.

The size and shape of the filler particles is another important issue. Would it be best to have large particles, small particles, or particles of varying sizes in a known distribution? Should the particles be spheres, rods, flakes or some other optimal shape? The next consideration is how the particles should be arranged throughout the base material. In the actual composite material the particles are randomly dispersed throughout the base material. What impact does the particle geometry have on the heat flow through the composite material? If we could control the placement of the particles, perhaps there is an optimal particle geometry.

The volume concentration of the particles is also important. It would certainly seem that the more filler particles, the higher the thermal conductivity, but there is a loading capacity for the base material. For each base material there is a point at which the filler particles cannot be incorporated into the base material while still having a well-mixed composite that retains the desired features of the base material. Also, it may be that adding a large number of filler particles does not significantly 
increase the thermal conductivity, so that the cost may outweigh the benefits. While we cannot address all of these questions in this dissertation, they provide a framework for our study.

In Chapter 2 we describe our composite material, which is a thermally conductive silicone. While all of our work will relate to this thermally conductive silicone, all of our results extend in a natural way to other thermally conductive composites. Throughout our discussions we will refer to this thermally conductive silicone as the composite silicone. We describe the components of the composite silicone, the base silicone polymer and the filler particles, and how we measure its thermal properties using a Holometrix Model Microflash. We also present some sample data from the Microflash. An understanding of the components of the composite silicone, along with the data collection process is necessary in formulating our model in Chapter 3. The resulting data will also guide our formulation of the parameter estimation problem in Chapter 6.

In Chapter 3 we present a mathematical model to describe the heat transfer through the composite silicone. This model takes into account the composition of the composite silicone and the data collection process as described in Chapter 2. We begin with a three dimensional model and then reduce that model to two dimensions for further study. We detail the use of Matlab's Partial Differential Equations Toolbox in obtaining numerical solutions to our two dimensional model. We also look at the design questions of particle size, particle distribution and particle placement. We describe several geometries with various particle sizes, particle distributions, and particle alignments. We present numerical results describing the effects of these geometries on heat transfer.

In Chapter 4 we outline the mathematical theory of homogenization. We show how homogenization can be used to find the "effective thermal conductivity" of our composite silicone. While we elect to use Matlab's Partial Differential Equations Toolbox and the method described in Chapter 3 for our study, we include this summary of homogenization theory for completeness. 
In Chapter 5 we show that the two dimensional mathematical model is well-posed. We show there exist unique, weak solutions to our mathematical model. Furthermore, these solutions are continuously dependent on the initial conditions, forcing function, and parameters.

In Chapter 6 we present two approaches to a parameter identification problem for our model. We formulate a constant parameter estimation problem and give some numerical results. We also describe a probability based parameter estimation problem where the parameters are viewed as realizations of random variables. This approach allows for uncertainty in the model parameters as well as the data. We then present the formulation for this approach as related to our model and give some numerical results.

In Chapter 7 we formally derive the sensitivity equations for our mathematical model. Furthermore, we numerically solve these equations and show that the model is more sensitive to some model parameters than others. These results provide some insight into the results of our parameter estimation problem in Chapter 6. Finally in Chapter 8, we summarize conclusions about the work presented here and suggest some directions for future work on this problem. 


\section{Chapter 2}

\section{Experimental Method}

In this chapter we describe the composition of the thermally conductive silicone and how it is produced in the laboratory. We then describe the Holometrix Model Microflash machine used to measure the thermal conductivity of the composite silicone. Finally, we present sample data from the data collection process.

\subsection{Composition of the Composite Silicone}

The silicone system used as the base for our composite silicone has a thermal conductivity of approximately $0.12 \mathrm{~W} / \mathrm{mK}$ (Watts per meter-Kelvin). The base silicone consists of a vinyl-functional siloxane (commonly referred to as a resin or polymer) and a hydride-functional siloxane (commonly called a crosslinker). When these two liquid components are cured the hydride adds to the double bond in the vinyl group to form a linkage

$$
\mathrm{SiCH}=\mathrm{CH}_{2}+\mathrm{H}-\mathrm{Si}--->\mathrm{SiCH}_{2}-\mathrm{CH}_{2} \mathrm{Si}
$$

which is sufficient to form a solid. For ease of reference, we refer to the silicone system as the silicone polymer. In hopes of creating a composite silicone with a higher thermal conductivity, filler particles with a greater thermal conductivity are added to the silicone polymer. A wide variety of filler particles, including aluminum particles, 
carbon fibers and diamond dust, can be added in varying concentrations. For our composite silicone, we use Grade 6 aluminum which has a thermal conductivity of $217 \mathrm{~W} / \mathrm{mK}$ and consider formulations with $25 \%, 35 \%$ and $45 \%$ filler by volume concentration. For the $25 \%$ by volume composite silicone, 45 grams of Grade 6 aluminum are added to 50 grams of silicone polymer. For the $35 \%$ and $45 \%$ filler by volume composite silicones, 73 grams and 111 grams of Grade 6 aluminum are added to 50 grams of silicone polymer. After all components are mixed together, the composite is a thick gray liquid and the higher the volume concentration of filler particles, the more viscous the liquid. The liquid composite is poured into a rectangular mold and cured for 30 minutes at $125^{\circ} \mathrm{C}$ (Celsius), and then for an additional 30 minutes at $150^{\circ} \mathrm{C}$.

Once the composite silicone is cured, samples are cut with a circular die, creating thin, cylindrical test pieces. Each test piece is weighed and its diameter and thickness measured. From these measurements, the volume and density of each test piece is computed. For the $25 \%$ by volume test pieces, the diameter of each test piece is approximately $12.86 \mathrm{~mm}$ (millimeters). The average thickness is $1.638 \mathrm{~mm}$ and the average weight is 0.302 grams. The diameter of each of the $35 \%$ by volume test pieces is approximately $12.82 \mathrm{~mm}$. The average thickness is $1.865 \mathrm{~mm}$ and the average weight is 0.385 grams. For the $45 \%$ by volume test pieces, the diameter of each test piece is approximately $12.86 \mathrm{~mm}$. The average thickness is $1.863 \mathrm{~mm}$ and the average weight is 0.418 grams. After all measurements are taken, each test piece is coated with graphite. We use a laser flash method, described in the next section, to measure the thermal conductivity of the samples. The graphite coating helps prevent reflection of the laser at the surface of the sample.

\subsection{Data Collection}

There are a variety of methods available to measure thermal conductivity. For our data collection we use a Holometrix Model Microflash. The Microflash uses a laser 
flash method which allows measurements to be taken at room temperature. The software used in conjunction with the machine is Microflash-RT, version 2.25.

A test piece is placed inside the Microflash machine and a short laser pulse is fired at one side of the test piece. On the opposite side of the test piece there is an infrared (IR) detector which measures temperature rise on this face of the sample due to the laser pulse. The laser firing and temperature measurements constitutes one trial and is repeated three times in the analysis of one test piece. A schematic diagram of the Microflash is depicted in Figure 2.1.

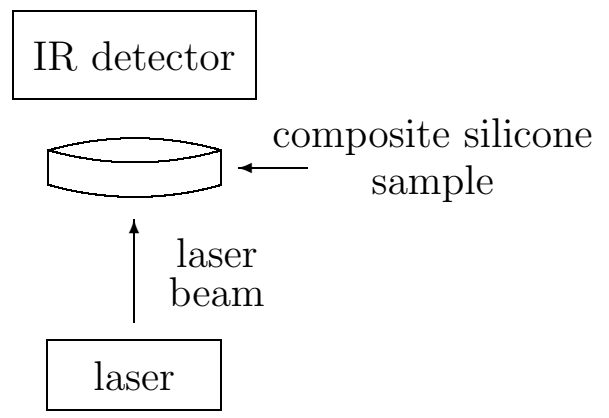

Figure 2.1: Schematic diagram of Holometrix Microflash

The Microflash software requires the user to input the thickness and the density of the test piece. It is important to note that the density of the composite silicone is not constant throughout the test piece. The value for the density of the test piece entered into the Microflash software is an effective density based on its overall mass and volume. Also, the test piece is assumed to be uniform in thickness, which, due to variations in the mold, may not true. In addition, a sample of known thermal conductivity must first be "tested" to calibrate the machine. We use a disk of pyroceram as our reference sample. Pyroceram is a Corning trademark for a series of crystalline ceramic materials made from powdered glasses. Our reference sample is Brand 9606 and has a thermal conductivity of $4 \mathrm{~W} / \mathrm{mK}$.

In each trial, the thermal diffusivity, $\alpha$, is calculated using Taylor analysis. The thermal diffusivity is calculated using a theoretical relationship determined by Clark 
and Taylor that relates the dimensionless parameter

$$
C=\frac{\alpha t_{50}}{d^{2}}
$$

and the time ratios

$$
\frac{t_{70}}{t_{30}}, \frac{t_{80}}{t_{40}}, \frac{t_{80}}{t_{20}},
$$

where $d$ is the thickness of the test piece and $t_{r}$ is the time it takes for the temperature to rise $r \%$ of the total temperature rise; e.g., $t_{50}$ is the time it takes for the temperature to rise to $50 \%$ of the peak value. The three values computed for $\alpha$ using each of the three time ratios (2.1) are then averaged. Further information on Taylor analysis can be found in [11, 15]. Thermal diffusivity can also be computed using the Cowan or Degiovanni method. See [15] for a summary of these two methods. Each of the three methods give approximately the same results.

The specific heat of the test piece is calculated by comparing it to the reference piece (pyroceram in our experiments). We will use sample to denote parameters specific to the test piece and ref to denote parameters specific to the reference piece. The specific heat is given by

$$
c_{p_{\text {sample }}}=\frac{\left(m c_{p} \Delta T\right)_{\text {ref }}}{(m \Delta T)_{\text {sample }}}
$$

where $m$ is the mass of the piece, $\Delta T$ is the temperature change at the IR detector and $c_{p}$ is the specific heat.

Using the calculated values for the diffusivity and specific heat, the thermal conductivity, $k$, is then computed using the relation

$$
k=\alpha \rho c_{p} .
$$

The mass density, $\rho$, is inputted prior to testing. This entire process is repeated three times and then averaged to give the thermal diffusivity, specific heat and thermal conductivity of the test piece.

Clearly this method works well for materials of uniform density, i.e., non-composites. However, since we are using composite materials we know that these results 
are only some measure of the "average" or "effective" thermal properties of the composite material. Thus it is difficult to answer design questions about the composite silicone based on test results we know are based only on these effective properties of the composite.

\subsection{Sample Data}

Analysis on a single test piece yields the diffusivity, the specific heat and the thermal conductivity of the sample based on an average of three trials. In addition to these three averaged values, the Microflash outputs the diffusivity, the specific heat and the thermal conductivity of each trial and the voltage at eight different times for each of the three trials. The eight times recorded are the time in milliseconds (msecs) to reach $0,20,30,40,50,70,80$, and 100 percent of the temperature rise (equivalently voltage rise, see below). The measurements at these times are used in Taylor analysis. Table 2.1 contains the output from each of the three trials in a test on one test piece of the $25 \%$ by volume composite silicone, along with the average diffusivity, specific heat and thermal conductivity.

It would be useful to know the total temperature rise for a given test piece, but the IR detector measures voltage rise, not temperature rise. However, if the temperature rise is small (we expect a 1-2 degree temperature rise in our experiments), the temperature rise is proportional to the voltage rise divided by the gain, i.e.

$$
\Delta T=\nu \frac{\Delta V}{G}
$$

where $\nu$ is an unknown constant of proportionality. Since right now we are only concerned with the total temperature rise, we will assume $\Delta V$ and $\Delta T$ refer to the total voltage rise and total temperature rise.

The laser energy as configured in the Microflash is approximately $7 \mathrm{~J}$. For our testing there is a $20 \%$ filter screen, so the actual laser energy, $Q$, is approximately $1.4 \mathrm{~J}$. Using this value we can solve $Q=m c_{p} \Delta T$ to get $\Delta T_{r e f}$, the temperature change 


\begin{tabular}{|c|c|c|c|c|c|c|}
\hline \multirow{2}{*}{$\begin{array}{l}\text { Voltage } \\
\text { Rise \% }\end{array}$} & \multicolumn{2}{|c|}{ Trial 1} & \multicolumn{2}{|c|}{ Trial 2} & \multicolumn{2}{|c|}{ Trial 3} \\
\hline & msecs & volts & msecs & volts & msecs & volts \\
\hline 0 & 0.000 & -0.043 & 0.000 & -0.043 & 0.000 & -0.055 \\
\hline 20 & 590.12 & 0.156 & 578.19 & 0.151 & 561.05 & 0.139 \\
\hline 30 & 713.43 & 0.256 & 725.45 & 0.248 & 703.01 & 0.236 \\
\hline 40 & 829.94 & 0.355 & 848.24 & 0.345 & 858.48 & 0.333 \\
\hline 50 & 1016.677 & 0.455 & 1051.934 & 0.442 & 1015.977 & 0.431 \\
\hline 70 & 1447.609 & 0.653 & 1366.658 & 0.636 & 1388.525 & 0.625 \\
\hline 80 & 1746.694 & 0.753 & 1650.390 & 0.733 & 1655.705 & 0.722 \\
\hline 100 & 3460.000 & 0.952 & 3036.667 & 0.927 & 3423.333 & 0.916 \\
\hline diffusivity & \multicolumn{2}{|c|}{0.00394} & \multicolumn{2}{|c|}{0.00363} & \multicolumn{2}{|c|}{0.00384} \\
\hline specific heat & \multicolumn{2}{|c|}{1.04702} & \multicolumn{2}{|c|}{1.07360} & \multicolumn{2}{|c|}{1.07151} \\
\hline conductivity & \multicolumn{2}{|c|}{0.58667} & \multicolumn{2}{|c|}{0.55374} & \multicolumn{2}{|c|}{0.58512} \\
\hline \multicolumn{3}{|c|}{ Average diffusivity } & \multicolumn{4}{|c|}{0.00381} \\
\hline \multicolumn{3}{|c|}{ Average specific heat } & \multicolumn{4}{|c|}{1.06405} \\
\hline \multicolumn{3}{|c|}{ Average thermal conductivity } & \multicolumn{4}{|c|}{0.57518} \\
\hline
\end{tabular}

Table 2.1: Data from test piece 25-1, 25\% by volume Grade 6 aluminum particles

for our pyroceram reference sample. The pyroceram sample has a mass of $0.6557 \mathrm{gm}$ (grams) and its specific heat is $0.775 \mathrm{~J} / \mathrm{gC}$ (Joules per gram-Celsius), and thus

$$
\Delta T_{\text {ref }}=\frac{1.4 \mathrm{~J}}{(0.6557 \mathrm{gm})(0.775 \mathrm{~J} / \mathrm{gC})}=2.755 \mathrm{C} .
$$

The total temperature rise of the pyroceram should be approximately the same in each trial. From here, we can substitute $\Delta T_{r e f}$ into equation (2.3) and solve for $\nu$, i.e.,

$$
\nu=2.755 \frac{G_{r e f}}{\Delta V_{\text {ref }}} .
$$

At this point, we can compute the temperature change for our test pieces in two different ways, either via equation (2.3) or by solving (2.2) for $\Delta T_{\text {sample, }}$,

$$
\Delta T_{\text {sample }}=\frac{\left(m c_{p} \Delta T\right)_{r e f}}{\left(m c_{p}\right)_{\text {sample }}} .
$$

We have all of the necessary values for both approaches. In Table 2.2, we summarize these values along with the value of $\Delta T$ for three test pieces using equations (2.3) and 
(2.5). The data is from one trial of test pieces $25-1$ ( $25 \%$ by volume filler particles), $35-2$ (35\% by volume filler particles) and $45-3$ ( $45 \%$ by volume filler particles). The Microflash was recalibrated using the pyroceram sample for each of these pieces.

\begin{tabular}{||c||c|c|c|c|c||c|c||}
\hline Name & $m$ & $c_{p}$ & $G$ & $\Delta V$ & $\nu$ & $\Delta T(2.3)$ & $\Delta T(2.5)$ \\
\hline \hline Pyroceram & 0.6557 & 0.775 & 0.496125 & 0.619 & 2.208 & \multicolumn{2}{|c}{} \\
\hline $25-1$ & 0.306 & 1.128 & 0.496125 & 0.995 & & 4.428 & 4.056 \\
\hline \hline Pyroceram & 0.6557 & 0.775 & 0.496125 & 8.006 & 0.171 & & \\
\hline $35-2$ & 0.388 & 1.145 & 0.496125 & 9.275 & & 3.197 & 3.151 \\
\hline \hline Pyroceram & 0.6557 & 0.775 & 0.496125 & 7.714 & 0.161 & & \\
\hline 45-3 & 0.394 & 1.215 & 0.496125 & 8.472 & & 2.749 & 2.925 \\
\hline \hline
\end{tabular}

Table 2.2: Temperature rise for three test pieces using equations (2.3) and (2.5)

We were expecting a temperature rise of 1-2 degrees, so our results seem reasonable. Our calculations of $\Delta T_{\text {sample }}$ are approximately the same for both calculations, which is reassuring. We would have expected the proportionality constant, $\nu$ to be the same in each calculation, which it is not. Instead it seems that the proportionality constant is dependent upon the voltage rise from the reference sample calibration and therefore unique to each test. The actual temperature rise could be measured using a thermocouple.

In the model we present in Chapter 3 we are able to measure temperature rise, not voltage rise, so this clearly introduces a source of error. In Chapter 6 we present a formulation for the parameter identification problem that allows us to represent the potential error in our data. Since we will only use simulated data in Chapter 6 for proof of concept, the translation of voltage rise to temperature rise does not require immediate attention, but it does warrant investigation in the future.

In order to have both inter-individual variability and intra-individual variability in our data, some test pieces were tested multiple times. Data was collected from all three composite silicone formulations. Tables 2.3, 2.4, and 2.5 report the average diffusivity, specific heat and thermal conductivity for some of the test pieces for the 
$25 \%, 35 \%$, and $45 \%$ by volume formulations. As the volume percentage increases, the average thermal conductivity increases as well. However, one might have expected the thermal conductivity values to be higher, given that the aluminum filler particles had a thermal conductivity of $217 \mathrm{~W} / \mathrm{mK}$.

\begin{tabular}{|c||c|c|c|}
\hline Sample No. & Diffusivity, $\mathrm{cm}^{2} / \mathrm{s}$ & Specific Heat, J/gK & Conductivity, W/mK \\
\hline \hline $25-1$ & 0.00381 & 1.064 & 0.575 \\
\hline $25-2$ & 0.00330 & 1.088 & 0.516 \\
\hline $25-3$ & 0.00367 & 1.039 & 0.543 \\
\hline $25-4$ & 0.00355 & 1.048 & 0.529 \\
\hline $25-5$ & 0.00352 & 1.082 & 0.545 \\
\hline $25-6$ & 0.00332 & 1.173 & 0.554 \\
\hline $25-7$ & 0.00299 & 1.208 & 0.511 \\
\hline $25-8$ & 0.00382 & 1.078 & 0.580 \\
\hline $25-9$ & 0.00431 & 1.060 & 0.647 \\
\hline
\end{tabular}

Table 2.3: Data from $25 \%$ by volume test pieces

\begin{tabular}{|c||c|c|c|}
\hline Sample No. & Diffusivity, $\mathrm{cm}^{2} / \mathrm{s}$ & Specific Heat, J/gK & Conductivity, W/mK \\
\hline \hline $35-1$ & 0.00422 & 1.12887 & 0.75077 \\
\hline $35-2$ & 0.00396 & 1.18239 & 0.74429 \\
\hline $35-3$ & 0.00409 & 1.16293 & 0.75262 \\
\hline $35-4$ & 0.00431 & 1.11351 & 0.75584 \\
\hline $35-5$ & 0.00417 & 1.11521 & 0.73505 \\
\hline $35-6$ & 0.00431 & 1.06210 & 0.72002 \\
\hline $35-7$ & 0.00423 & 1.07061 & 0.71761 \\
\hline $35-8$ & 0.00417 & 1.15571 & 0.76976 \\
\hline $35-9$ & 0.00451 & 1.16818 & 0.81503 \\
\hline
\end{tabular}

Table 2.4: Data from 35\% by volume test pieces 


\begin{tabular}{|c||c|c|c|}
\hline Sample No. & Diffusivity, $\mathrm{cm}^{2} / \mathrm{s}$ & Specific Heat, J/gK & Conductivity, W/mK \\
\hline \hline $45-1$ & 0.00524 & 1.12605 & 1.01195 \\
\hline $45-2$ & 0.00521 & 1.13109 & 1.02672 \\
\hline $45-3$ & 0.00544 & 1.19630 & 1.11223 \\
\hline $45-4$ & 0.00516 & 1.05451 & 0.94062 \\
\hline $45-5$ & 0.00527 & 1.03203 & 0.93413 \\
\hline $45-6$ & 0.00526 & 1.06907 & 0.97309 \\
\hline $45-7$ & 0.00527 & 1.03485 & 0.94248 \\
\hline $45-8$ & 0.00523 & 1.10749 & 0.99055 \\
\hline $45-9$ & 0.00540 & 1.26932 & 1.17448 \\
\hline
\end{tabular}

Table 2.5: Data from $45 \%$ by volume test pieces 


\section{Chapter 3}

\section{A Model for Heat Transfer through a Composite Silicone}

In this chapter we present a three dimensional model describing the behavior of heat transfer through a composite silicone based on the Holometrix Microflash method described in Chapter 2. For proof of concept we reduce the model to a two dimensional version and present numerical solutions to this model. At the end of the chapter we study the influence of different composite geometries on the heat transfer process.

\subsection{Three Dimensional Model}

Since the fundamental process of our problem is heat transfer through the composite silicone, the foundation of our model is clearly the transient heat equation. While keeping the composition of the composite silicone and the data collection process in mind, it is necessary to make a few simplifying assumptions. First, we assume all heat from the heat source (the laser) flows through the composite silicone and into the heat sink (the IR detector), as depicted in Figure 3.1. Second, since the composite silicone slice is very thin, we assume there is no heat loss through the sides. Thus in our model we assume the sides of the composite silicone are insulated. We use a flux boundary condition to describe the heating on one side of the composite silicone due to the laser. On the other side of the composite silicone, where the IR detector 
is located, we use Newton cooling to describe the boundary condition since that face of the composite silicone touches the surrounding air.

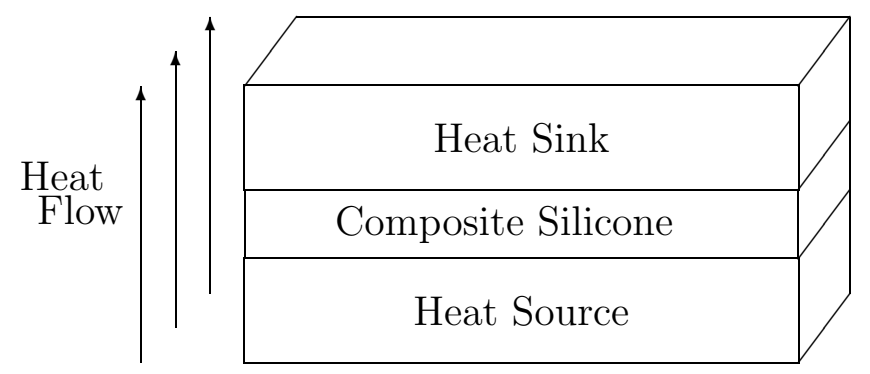

Figure 3.1: Three dimensional heat transfer model

We call the three dimensional composite silicone slice $\bar{\Omega}$ and set $\partial \bar{\Omega}=\Gamma_{1} \cup \Gamma_{2} \cup \Gamma_{3}$ where $\Gamma_{1}$ is the boundary between the composite silicone and the heat source, $\Gamma_{2}$ is the boundary between the heat sink and the composite silicone, and $\Gamma_{3}$ represents all remaining (lateral) boundaries as depicted in Figure 3.2. The parameters $k, \rho$, and $c_{p}$, the thermal conductivity, mass density and specific heat respectively, will all be spatially dependent having one constant value in the solid (e.g., aluminum) filler particles and another constant value in the silicone polymer. In actuality, the silicone polymer is itself a composite comprised of the polymer and the crosslinker as described in Section 2.1. However, the chemical reaction cures the two components together so that they are essentially one material. Due to the nature of the linkage it would be impossible to separate the two components in our model, and so we treat them as one material with constant parameter values.

It is clear that the filler particles will not be uniform in size, and will most likely be randomly dispersed throughout the base silicone polymer. However, it will be necessary to know the size of each particle and the arrangement of the particles in order to determine the value of $k, \rho$, and $c_{p}$ at a particular point in the composite silicone. To facilitate our modeling, we will assume the filler particles are spheres comprising the appropriate volume percent of the composite silicone and that there 


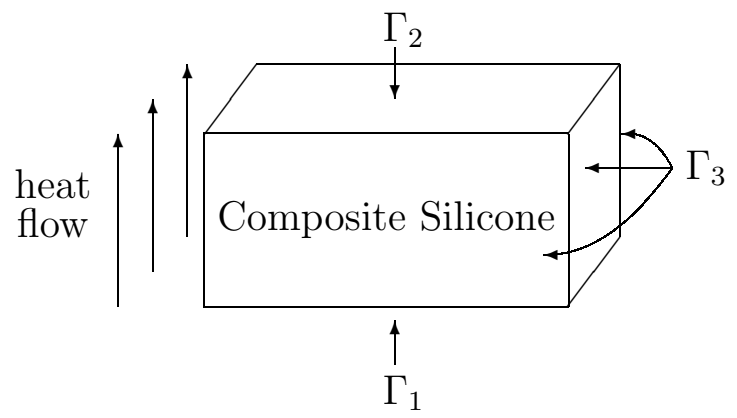

Figure 3.2: Three dimensional composite silicone boundaries

is a known particle arrangement.

We will call the ambient temperature $T_{\infty}$ and the initial temperature of the composite silicone $u_{0}$. We denote the Newton cooling constant by $h$, and define $S_{0}(t)$ to be the flux due to the heat source. Thus if $u(t, \bar{z})$ is the composite silicone temperature at a given time $t$ and coordinate $\bar{z}$, we have the following system describing our experiment:

$$
\left\{\begin{array}{l}
\rho(\bar{z}) c_{p}(\bar{z}) \frac{\partial u}{\partial t}(t, \bar{z})=\nabla \cdot(k(\bar{z}) \nabla u(t, \bar{z})) \\
\left.\frac{\partial u}{\partial n}(t, \bar{z})\right|_{\Gamma_{1}}=\left.h\left(T_{\infty}-u(t, \bar{z})\right)\right|_{\Gamma_{1}} \\
\left.\frac{\partial u}{\partial n}(t, \bar{z})\right|_{\Gamma_{2}}=S_{0}(t) \\
\left.\frac{\partial u}{\partial n}(t, \bar{z})\right|_{\Gamma_{3}}=0 \\
u(0, \bar{z})=u_{0}(\bar{z})
\end{array}\right.
$$

for $t \in[0, T]$ and $\bar{z} \in \bar{\Omega}$.

Despite the simplifying assumptions we have described, we believe the three dimensional model is a reasonable representation of the thermal process taking place during the Microflash experiments. However, for our initial study of the problem we have elected to reduce the three dimensional model to a two dimensional model. In the next section we describe the two dimensional model and its relationship to the three dimensional model we have just presented. 


\subsection{Two Dimensional Model}

The two dimensional model can be thought of as a very thin interior slice (in the direction of the heat flow) of the three dimensional model as depicted in Figure 3.3. We assume the composite silicone is significantly thicker in the direction normal to the slice compared to the slice itself, so all heat will flow directly through the composite silicone with negligible lateral dissipation. If we recall the test pieces described in Section 2.1, the diameters of the pieces are much greater than the thickness of the piece, so our assumption is reasonable. Furthermore we assume the geometry of the composite silicone is uniform normal to the slice, provided we are in the center of the composite silicone away from lateral edges. Thus the two dimensional model heat flow should closely resemble the three dimensional heat flow.

We will continue to assume the sides of the composite silicone are insulated. The boundary at the heat source will be a Neumann boundary condition given by the heat flux due to the laser and the boundary at the heat sink will still be described by Newton cooling. Reducing the three dimensional model equations in (3.1) to fit the two dimensional model, we obtain

$$
\begin{cases}\rho(z) c_{p}(z) \dot{u}(t, z)=\nabla \cdot(k(z) \nabla u(t, z)) & \\ \left.k(z) \frac{\partial u}{\partial n}(t, z)\right|_{\gamma_{4}}=S_{0}(t) & (\text { source }) \\ \left.k(z) \frac{\partial u}{\partial n}(t, z)\right|_{\gamma_{2}}=\left.h\left(T_{\infty}-u(t, z)\right)\right|_{\gamma_{2}} & (\text { sink }) \\ \left.k(z) \frac{\partial u}{\partial n}(t, z)\right|_{\gamma_{1}}=0 & \\ \left.k(z) \frac{\partial u}{\partial n}(t, z)\right|_{\gamma_{3}}=0 & \\ u(0, z)=\Upsilon(z) & \end{cases}
$$

for $z \in \Omega=\left[-\frac{c_{1}}{2}, \frac{c_{1}}{2}\right] \times\left[-\frac{c_{2}}{2}, \frac{c_{2}}{2}\right]$ and $t \in[0, T]$, where $\dot{u}=\frac{\partial u}{\partial t}$. We will assume $c_{1}, c_{2}$, and $T$ are finite, positive constants. Let $\partial \Omega=\gamma_{1} \cup \gamma_{2} \cup \gamma_{3} \cup \gamma_{4}$ where

$$
\begin{aligned}
& \gamma_{1}(s)=\left\{\left(\frac{c_{1}}{2}, s\right): s \in\left[-\frac{c_{2}}{2}, \frac{c_{2}}{2}\right]\right\}, \\
& \gamma_{2}(s)=\left\{\left(s, \frac{c_{2}}{2}\right): s \in\left[-\frac{c_{1}}{2}, \frac{c_{1}}{2}\right]\right\},
\end{aligned}
$$




$$
\begin{aligned}
& \gamma_{3}(s)=\left\{\left(-\frac{c_{1}}{2}, s\right): s \in\left[-\frac{c_{2}}{2}, \frac{c_{2}}{2}\right]\right\}, \text { and } \\
& \gamma_{4}(s)=\left\{\left(s,-\frac{c_{2}}{2}\right): s \in\left[-\frac{c_{1}}{2}, \frac{c_{1}}{2}\right]\right\},
\end{aligned}
$$

as depicted in Figure 3.4. We define $\Omega_{s} \subset \Omega$ to be the region occupied by the silicone and $\Omega_{p} \subset \Omega$ to be the region occupied by the particles. Note $\Omega_{s} \cap \Omega_{p}=\emptyset$ and $\Omega_{s} \cup \Omega_{p}=\Omega$.

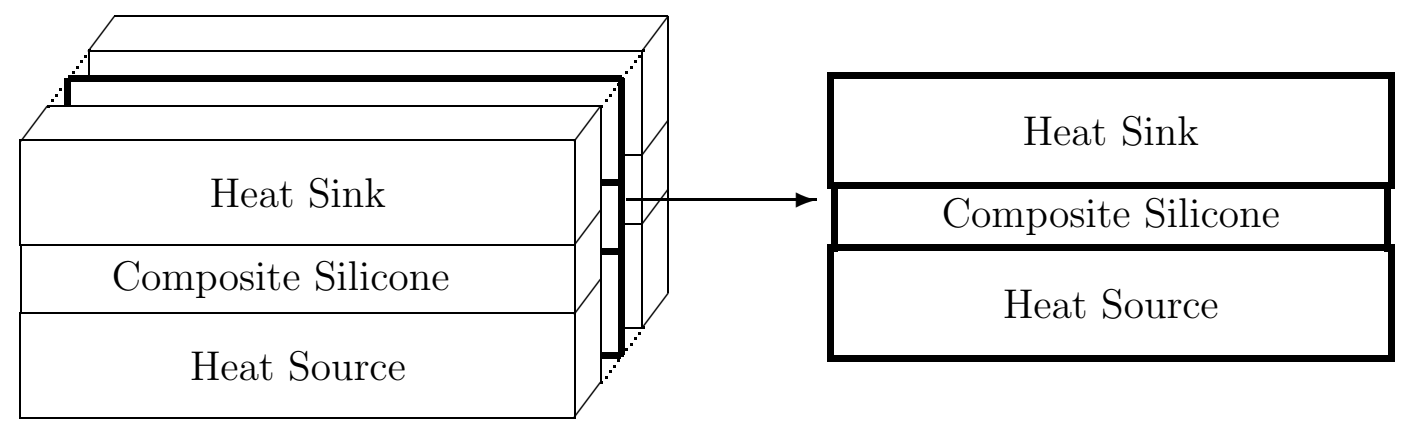

Figure 3.3: Two dimensional heat transfer model

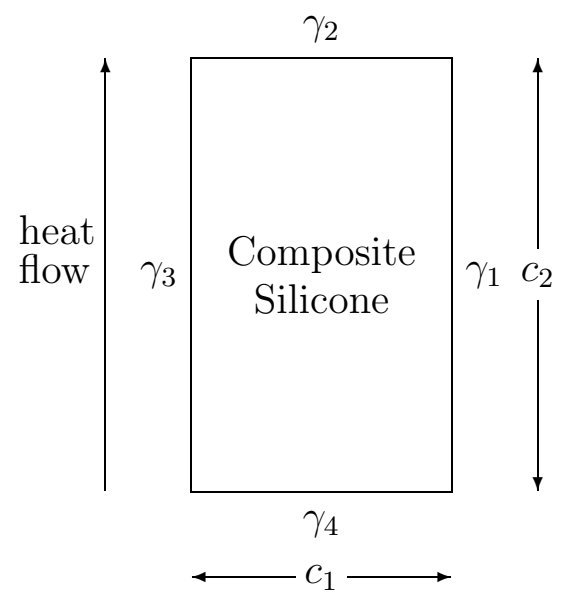

Figure 3.4: Two dimensional composite silicone slice 
As in the three dimensional model, it is important to note that $\rho, c_{p}$ and $k$ are all spatially dependent. They will have one value in the silicone polymer and another value in the filler particles. For the two dimensional case, we will assume all particles are circles in a known, although not necessarily uniform, particle arrangement.

\subsection{Matlab PDE Toolbox Solutions}

One benefit of using the two dimensional model is that we can use Matlab's Partial Differential Equation Toolbox (PDE Toolbox) to solve (3.2). Matlab's PDE Toolbox can solve two dimensional parabolic partial differential equations. In order to do this we must first define a geometry for the composite silicone. We initially assume all aluminum filler particles in the three dimensional model are spheres of uniform diameter, represented in the two dimensional model by circles of uniform diameter. Since we are using Grade 6 aluminum particles we will use the mean diameter of the volume distribution provided by the aluminum supplier, $24.14 \mu$ (microns), as the diameter of each particle. We will concentrate on the $25 \%$ by volume composite silicone, but all ideas presented here extend in a natural way to the $35 \%$ and $45 \%$ by volume composite silicones.

The silicone polymer used as the base for the composite silicone wets well, meaning it forms a thin film around each of the particles. The film formed around each particle is estimated to be approximately 50 angstroms. Hence, we assume each particle is separated by a minimum distance of 0.01 microns and that no particles touch the boundary of the composite silicone slice. We will assume initially all particles are arranged in a uniform geometry, i.e., the particles are uniformly spaced and aligned in rows. The area of the particles constitutes $25 \%$ of the total area of the composite silicone slice which is $321.5 \mu$ wide and $1638 \mu$ high, i.e., $c_{1}=321.5$ and $c_{2}=1638$ in the two dimensional model of the previous section. Figure 3.5 depicts the $321.5 \mu$ by $1638 \mu$ composite silicone slice with 288 circles of diameter $24.14 \mu$ representing the $25 \%$ by area aluminum particles used by the PDE Toolbox as the geometry for our 
composite silicone.

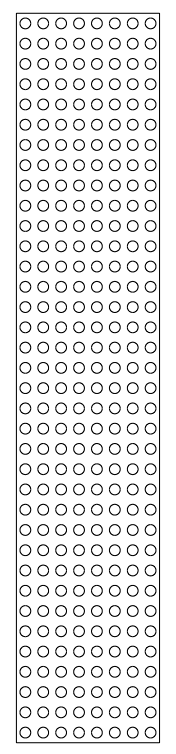

Figure 3.5: Two dimensional composite silicone geometry

Recall $k, \rho$ and $c_{p}$ are all spatially dependent. In order to differentiate between the value of each parameter in the silicone versus the aluminum particle we will quantify this variability as follows: for $z \in \Omega$, the value for each parameter is given by:

$$
\begin{aligned}
& k(z)= \begin{cases}k_{s} & z \in \Omega_{s} \\
k_{p} & z \in \Omega_{p}\end{cases} \\
& \rho(z)= \begin{cases}\rho_{s} & z \in \Omega_{s} \\
\rho_{p} & z \in \Omega_{p}\end{cases}
\end{aligned}
$$

and

$$
c_{p}(z)= \begin{cases}c_{p_{s}} & z \in \Omega_{s} \\ c_{p_{p}} & z \in \Omega_{p} .\end{cases}
$$

where $k_{s}, k_{p}, \rho_{s}, \rho_{p}, c_{p_{s}}$, and $c_{p_{p}}$ are all finite constants. Note $k, \rho$, and $c_{p}$ are each functions from $\Omega$ to $\mathbb{R}$ and each is piecewise constant. 
We assume the composite silicone was initially at the ambient temperature and the temperature was uniform throughout the sample. Thus we set $\Upsilon(z)=T_{\infty}$. We choose the Newton constant to represent air cooling as we have in our model. The exact model parameters used in the simulations reported here are in Table 3.1. In the table, $\mathrm{g} / \mathrm{cm}^{3}$ is grams per cubic centimeter and $\mathrm{J} / \mathrm{gK}$ is Joules per gram-Kelvin.

The source flux will approximate the energy in the laser pulse. The laser energy as configured in the Microflash is approximately 7 J. For our testing there is a $20 \%$ filter screen, so the actual laser energy is approximately $1.4 \mathrm{~J}$. In addition, since the pieces are graphite coated, Holometrix estimates there is an additional $20 \%$ energy loss. Given that the length of the laser pulse is 330 microseconds and the diameter of the laser is $10 \mathrm{~mm}$, we calculate the flux due to the laser pulse to be $S_{\ell}=4.32 \times 10^{7} \mathrm{~W} / \mathrm{m}^{2}$ (Watts per meter squared). Thus the source flux is given by

$$
S_{0}(t)= \begin{cases}S_{\ell} & 0 \leq t \leq t_{p} \\ 0 & t_{p}<t\end{cases}
$$

where $t_{p}=0.000330$ seconds. While we believe this is a high estimate, it is sufficient to begin our study.

\begin{tabular}{|c|c|}
\hline$k_{s}$ & $0.12 \mathrm{~W} / \mathrm{mK}$ \\
\hline$k_{p}$ & $217 \mathrm{~W} / \mathrm{mK}$ \\
\hline$\rho_{s}$ & $1 \mathrm{~g} / \mathrm{cm}^{3}$ \\
\hline$\rho_{p}$ & $2.7 \mathrm{~g} / \mathrm{cm}^{3}$ \\
\hline$c_{p_{s}}$ & $1.55 \mathrm{~J} / \mathrm{gK}$ \\
\hline$c_{p_{p}}$ & $0.90 \mathrm{~J} / \mathrm{gK}$ \\
\hline$T_{\infty}$ & $296.15 \mathrm{~K}$ \\
\hline$h$ & 350 \\
\hline$S_{\ell}$ & $4.32 \times 10^{7} \mathrm{~W} / \mathrm{m}^{2}$ \\
\hline
\end{tabular}

Table 3.1: Model parameters

We can compute the thermal diffusivity of the silicone, $\alpha_{s}$, and thermal diffusivity 
of the particles, $\alpha_{p}$ using the relation

$$
\alpha=\frac{k}{\rho c_{p}}
$$

Using the values in Table 3.1, we note that

$$
\alpha_{s}=\frac{0.12 \mathrm{~W} / \mathrm{mK}}{\left(1 \mathrm{~g} / \mathrm{cm}^{3}\right)(1.55 \mathrm{~J} / \mathrm{gK})}=0.000774 \mathrm{Wcm}^{2} / \mathrm{J}=0.000774 \mathrm{~cm}^{2} / \mathrm{s}
$$

and

$$
\alpha_{p}=\frac{217 \mathrm{~W} / \mathrm{mK}}{\left(2.7 \mathrm{~g} / \mathrm{cm}^{3}\right)(0.9 \mathrm{~J} / \mathrm{gK})}=0.893 \mathrm{Wcm}^{2} / \mathrm{J}=0.893 \mathrm{~cm}^{2} / \mathrm{s} .
$$

Thus we expect the thermal diffusivity of our composite to be between $0.000774 \mathrm{~cm}^{2} / \mathrm{s}$ and $0.893 \mathrm{~cm}^{2} / \mathrm{s}$. The thermal diffusivity values from the Microflash experiments given in Tables 2.3 - 2.5 all lie within this interval.

We solve (3.2) at the eight times in milliseconds given in Table 3.2. These times were chosen to match the data in Section 2.3. While the actual times vary from trial to trial, the difference is negligible.

\begin{tabular}{|c|c|c|c|c|c|c|c|}
\hline$t_{1}$ & $t_{2}$ & $t_{3}$ & $t_{4}$ & $t_{5}$ & $t_{6}$ & $t_{7}$ & $t_{8}$ \\
\hline 0 & 590.12 & 713.43 & 829.94 & 1016.677 & 1447.609 & 1746.964 & 3460.0 \\
\hline
\end{tabular}

Table 3.2: Times in milliseconds

In order to solve (3.2) using the PDE Toolbox we must enter the boundary conditions, the PDE coefficients and the composite silicone geometry. The PDE Toolbox generates a triangular mesh using the Delaunay triangulation algorithm and numerically solves the PDE using the finite element method with linear elements (the only type of elements the PDE Toolbox uses). The PDE Toolbox automatically defines the mesh, although the user has the option to refine the mesh. See [20] for further information about Matlab's Partial Differential Equation Toolbox. Figure 3.6 depicts the solution to (3.2) using the parameters specified in Table 3.1 at the times in Table 3.2. In this example the finite element method uses 15, 442 linear elements. 
In Figure 3.6, the heat flows from left to right. The heat source is at the edge where $y=-819$ and the heat sink is at the edge where $y=819$. The edges $x= \pm 160.75$ are the insulated sides. The heat from the heat source dissipates through the composite silicone slice towards the heat sink as time progress, and by $t_{8}$ it is nearing equilibrium.

Figure 3.7 exhibits a portion of the solution at time $t_{2}$. The segment is a cross section between $y=-275$ and $y=-125$. In this close up section we are able to see detail that is not clear in the graphs of the solution over the full composite silicone slice. Recall the particles are uniformly arranged in rows (see Figure 3.5). The ridges that appear correspond to the particles in the composite. Figure 3.8 is a graph of the temperature at the $\gamma_{2}$ boundary at time $t_{2}$ and this temperature profile suggests the influence of the particles. There are eight "peaks" in the temperature profile, and recall there are eight columns of particles, thus each peak is correlated to a column of particles. The mesh generated by the PDE Toolbox is not uniform, which is why the profile is jagged. Based on these observations, we conclude that the particles have a significant impact on the heat flow through the composite silicone. 

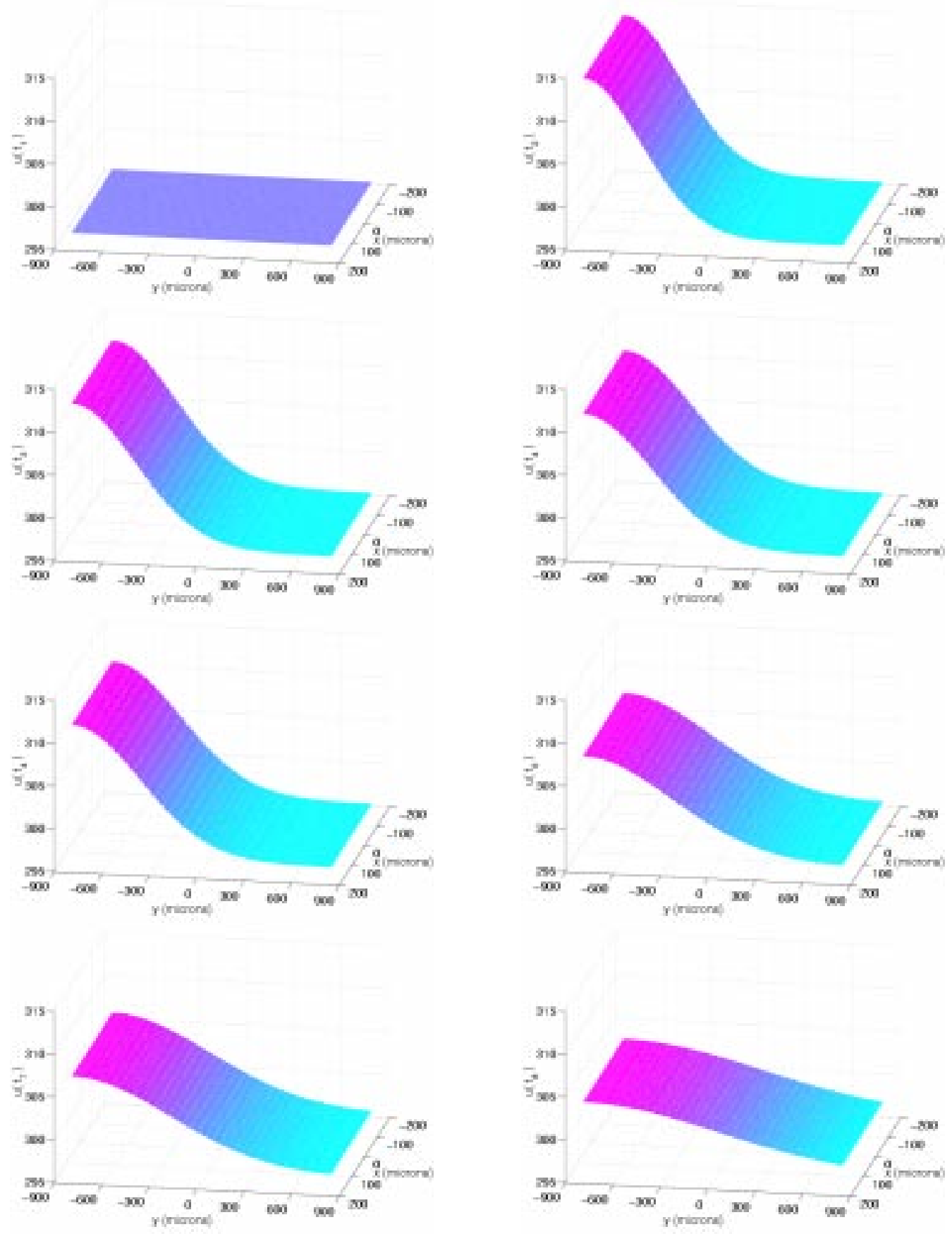

Figure 3.6: PDE Toolbox solution at $t_{1}, t_{2}, \cdots, t_{8}$ 


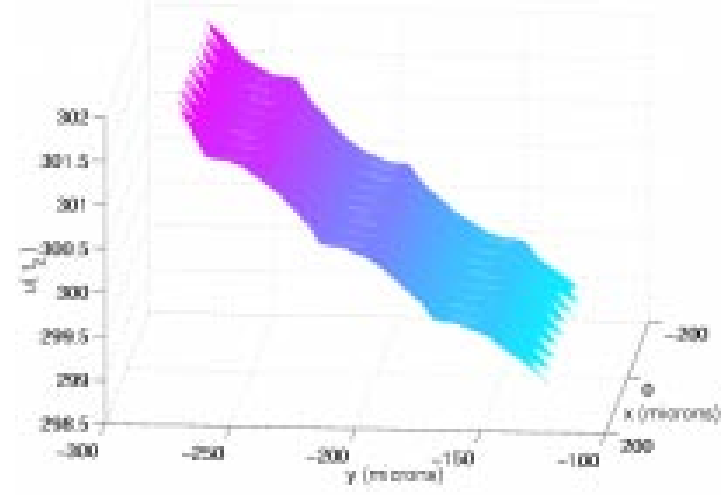

Figure 3.7: Close up segment of solution at $t_{2}$

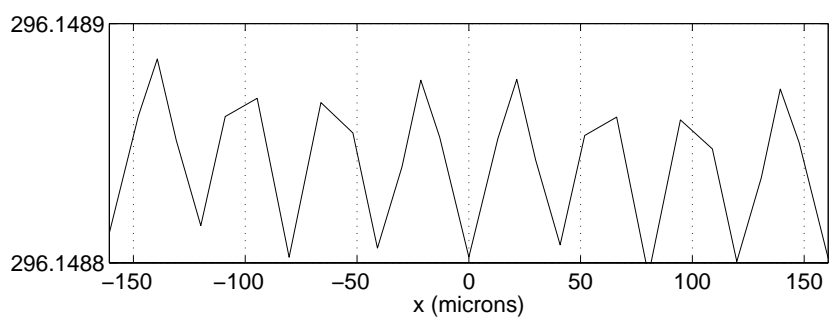

Figure 3.8: Temperature at $\gamma_{2}$ boundary at time $t_{2}$

\subsection{Different Particle Geometries}

Based on the observations in the previous section, we take a further look at the impact of the particle geometry on the heat transfer process. To illustrate the effect of geometry, we concentrate on the limit equilibrium or steady-state heat flow through the sample. We will therefore solve the steady state heat equation described in (3.3) below over a two dimensional composite slice that is $508 \mu$ wide by $50 \mu$ high, as depicted in Figure 3.9. We use Dirichlet boundary conditions at both the interface between the heat sink and the composite, $\gamma_{2}$, and the interface between the heat 
source and the composite interface, $\gamma_{4}$. In other words both the heat source and the heat sink maintain a constant temperature, given in degrees Kelvin (K). We assume the lateral sides, $\gamma_{1}$ and $\gamma_{3}$, are insulated.

$$
\begin{cases}\nabla \cdot(\nabla k \nabla u)=0 & \\ \left.u\right|_{\gamma_{2}}=375 & \\ \left.u\right|_{\gamma_{4}}=400 & (\text { source }) \\ \left.\frac{\partial u}{\partial n}\right|_{\gamma_{1}}=0 & \\ \left.\frac{\partial u}{\partial n}\right|_{\gamma_{3}}=0 & \text { sink })\end{cases}
$$

As in our model in Section 3.2, the thermal conductivity, $k$, is spatially dependent. We will assume it has one constant value on the base polymer of the composite, which we again call $k_{s}$, and another value on the filler particles, which we again call $k_{p}$. For our study of the particle geometries, we set $k_{s}=0.38 \mathrm{~W} / \mathrm{mK}$ and $k_{p}=1000 \mathrm{~W} / \mathrm{mK}$.

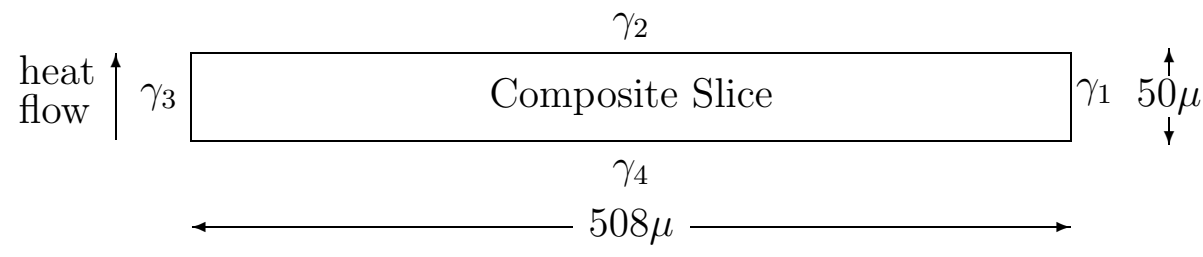

Figure 3.9: Two dimensional composite slice

In each particle geometry the particles comprise approximately $50 \%$ of the area of the two dimensional composite slice. We will concentrate on three categories of particle arrangements: uniform, shifted, and random. We will consider geometries with one size of particles and geometries with particles of multiple sizes. In the uniform geometry, all particles are arranged in straight rows and columns. In the shifted geometry, the particles are in staggered rows. Random geometries are generated by a random number generator selecting the center of each particle, one particle at a time. After one particle's center is chosen, its placement is checked against the other 
existing particles to ensure it does not overlap an existing particle. Additionally, it must be a minimum distance from the boundaries of the composite slice and the existing particles (we assume the base polymer wets well, as our silicone polymer does). If it does not satisfy these requirements, a different center is chosen. This process is repeated until the particle satisfies these conditions. Then the next particle is placed. If there are particles of differing sizes to be placed, the largest particles are placed first, then the next largest, and so on. The smallest particles are placed last.

Figures 3.10 - 3.23 depict the 14 different particle geometries we studied. All the particles are represented as circles. Due to the resolution of the graphs, the smallest sized particles appear as dots. Also, in some of the geometries it appears the particles are touching, but in each geometry, the minimum distance requirement between particles and the boundary is satisfied. All units in Figures 3.10-3.23 are microns.

Figures 3.10, 3.11, and 3.12 exhibit three examples of uniform geometries of same sized particles. In Figure 3.10 all particles have radius $22.5 \mu$, in Figure 3.11 all particles have radius $11.5 \mu$, and in Figure 3.12 all particles have radius $6 \mu$. Figures 3.13 and 3.14 depict two examples of uniform geometries with different sized particles. In Figure 3.13 there are two particles sizes, one is radius $22.5 \mu$, the other radius $11.5 \mu$, and they are in a $1: 4$ distribution. The radii of the three particles in Figure 3.14 are $22.5 \mu, 11.5 \mu$ and $0.625 \mu$ and the particles are in a $4: 16: 39$ distribution.

For the shifted geometry, we considered geometries of same sized particles. Figures 3.15 and 3.16 display two examples of shifted geometries with particles of radius $11.5 \mu$ and $6 \mu$ respectively. Each of these is derived from the uniform geometry by shifting alternate rows.

We consider random geometries with same sized particles, and random geometries with multiple sized particles. Figures 3.17 and 3.18 exhibit two different random geometries for eight particles of radius $22.5 \mu$. Figures 3.19 and 3.20 depict two different random geometries for 31 particles of radius $11.5 \mu$. For the random geometry with different sized particles we took particles of radius $22.5,11.5$ and 0.625 in a $1: 3: 7$ 
distribution. Three examples of this are in Figures 3.21, 3.22, and 3.23.

Since we are ultimately interested in the behavior of the model at the interface between the composite and the heat sink, we solve (3.3) using each of the different geometries in Figures 3.10 - 3.23 and measure the flux at the $\gamma_{2}$ boundary. Figure 3.24 depicts the solution to (3.3) and the flux at the heat sink using the composite geometry with uniformly arranged particles of radius $11.5 \mu$ in Figure 3.11. Note the ridges in the solution correspond to the location of the particles. Also note the 16 peaks in the flux correspond with the 16 columns of particles in the geometry.

In Figure 3.25, we solve (3.3) using the shifted geometry with particles of radius $11.5 \mu$ in Figure 3.15. Again, the ridges in the solution correspond to the placement of the particles in the composite. Note also that the flux is slightly different from the flux due to the aligned uniform geometry.

In Figure 3.26, we solve (3.3) using the random geometry with particles of radius 11.5 $\mu$ in Figure 3.19. In the solution it is clear the ridges are more random, due to the random particle placement. Also, the peaks in the flux appear to match the areas where there was a particle close to the $\gamma_{2}$ boundary.

Finally, Figure 3.27, depicts the solution to (3.3) when solved using the random geometry with three different sized particles depicted in Figure 3.21. Here the largest ridges in the solution and the large peaks in the flux correspond to the location of the location of the largest particles.

From these four representative solutions it is clear that the geometry impacts the heat transfer through the composite. From the graphs of the flux in these examples it appears that the flux in the uniform geometry is slightly larger than the flux in the shifted geometry, but less than the flux in either of the random geometries. However, in order to carefully compare the impact of the different geometries on the heat transfer process, we need to quantify the flux at the interface between the composite and the heat sink.

In Table 3.3 we give the norm of the flux at the interface between the composite and the heat sink, measured in the $L^{1}$ norm, the $L^{2}$ norm and the supremum norm, 


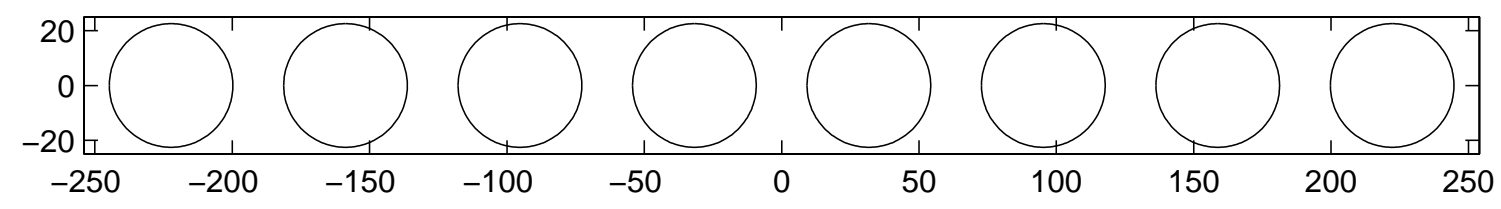

Figure 3.10: Uniform geometry, 8 particles of radius 22.5

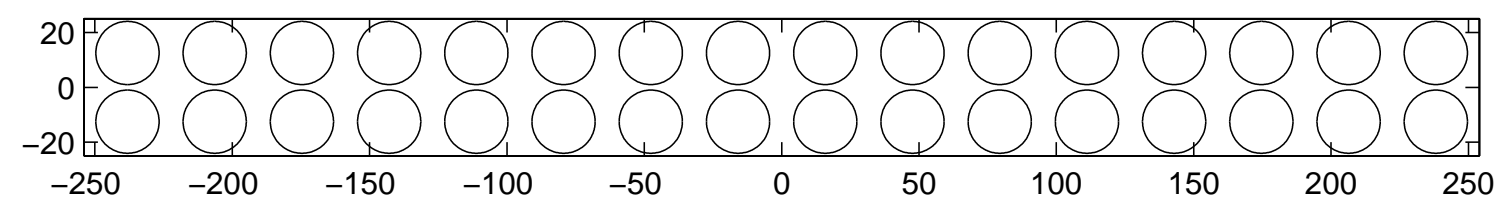

Figure 3.11: Uniform geometry, 32 particles of radius 11.5

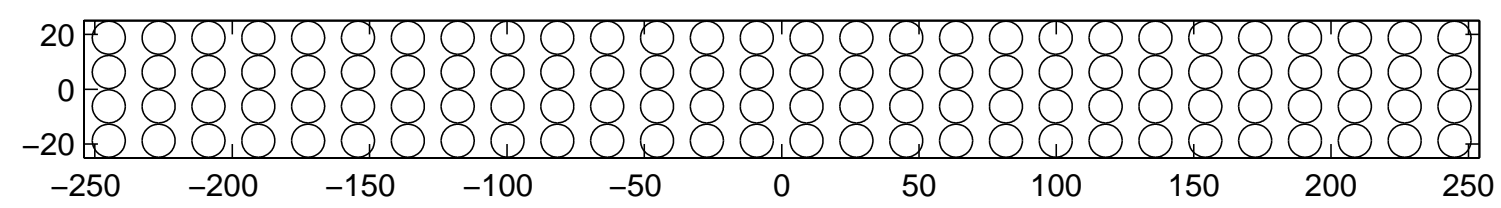

Figure 3.12: Uniform geometry, 112 particles of radius 6

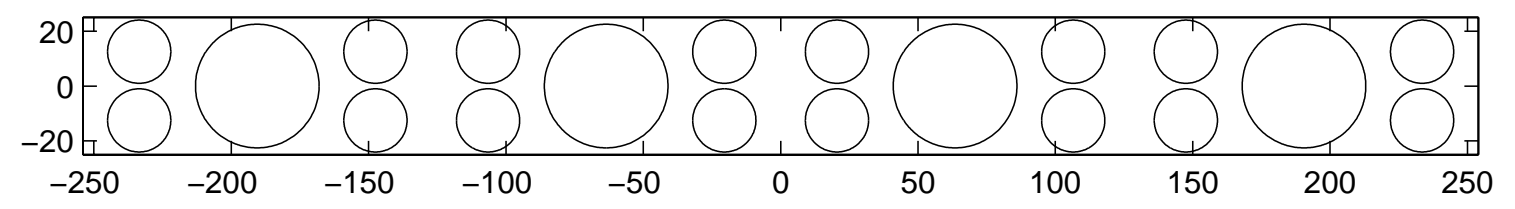

Figure 3.13: Uniform geometry, 20 particles particles of radius 22.5 and 11.5 satisfying a 1:4 distribution

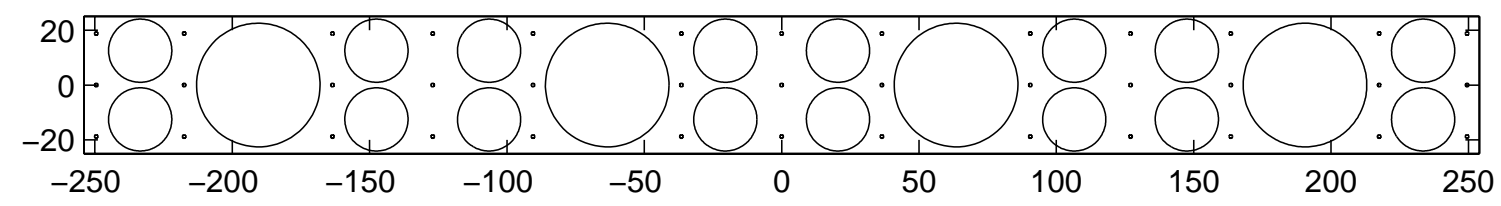

Figure 3.14: Uniform geometry, 49 particles particles of radius 22.5, 11.5, and 0.625 satisfying a 4:16:39 distribution 


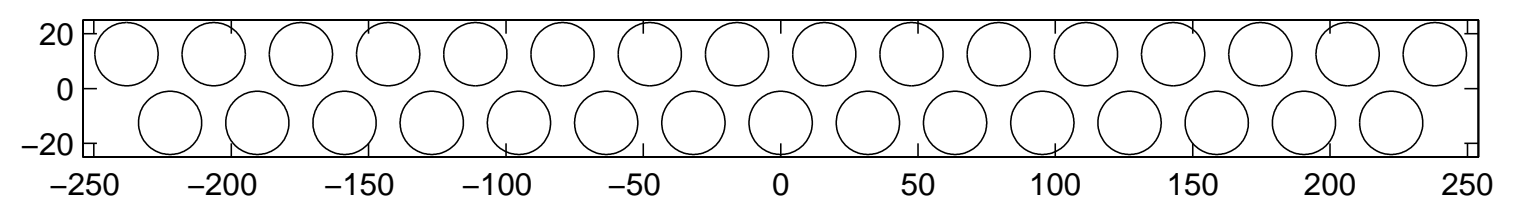

Figure 3.15: Shifted geometry, 31 particles of radius 11.5

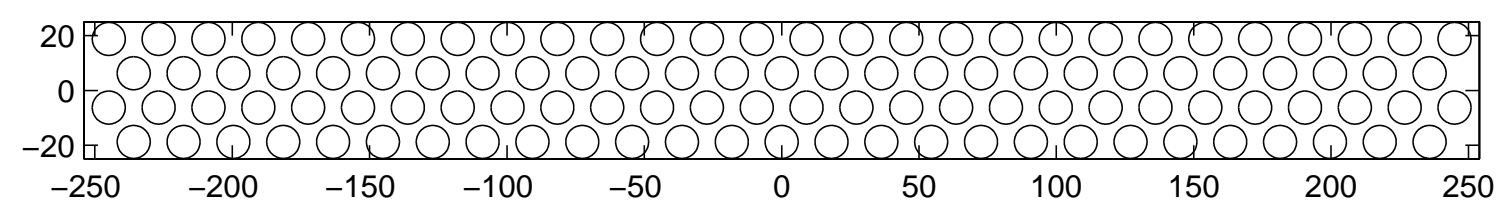

Figure 3.16: Shifted geometry, 110 particles of radius 6

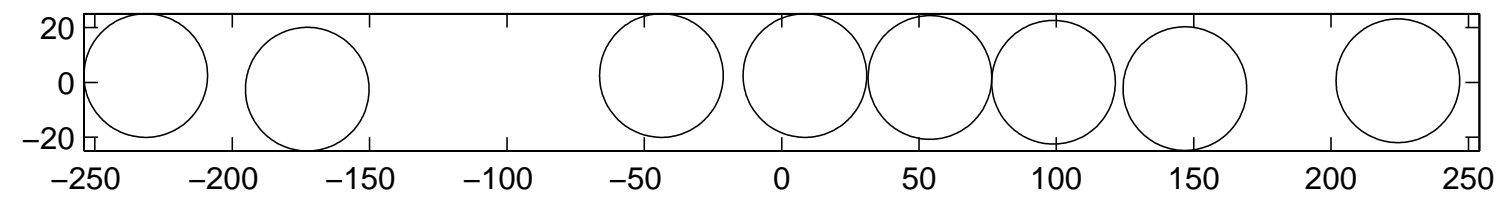

Figure 3.17: Random geometry, 8 particles particles of radius 22.5

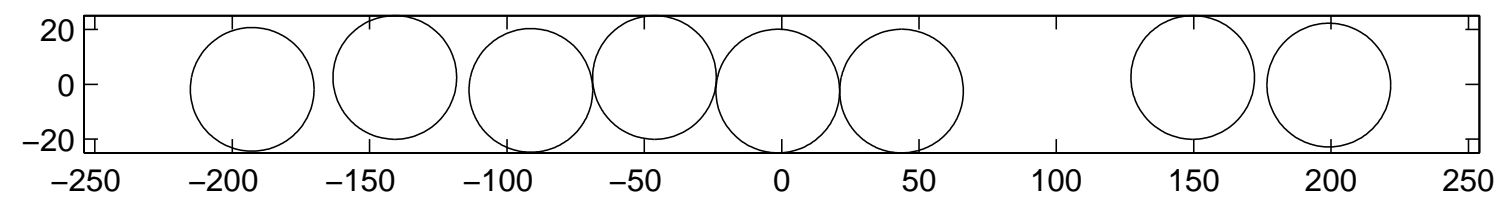

Figure 3.18: Random geometry, 8 particles particles of radius 22.5

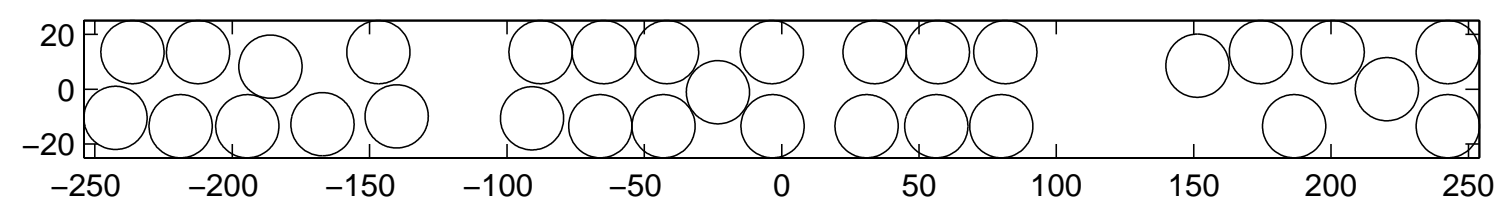

Figure 3.19: Random geometry, 31 particles particles of radius 11.5 


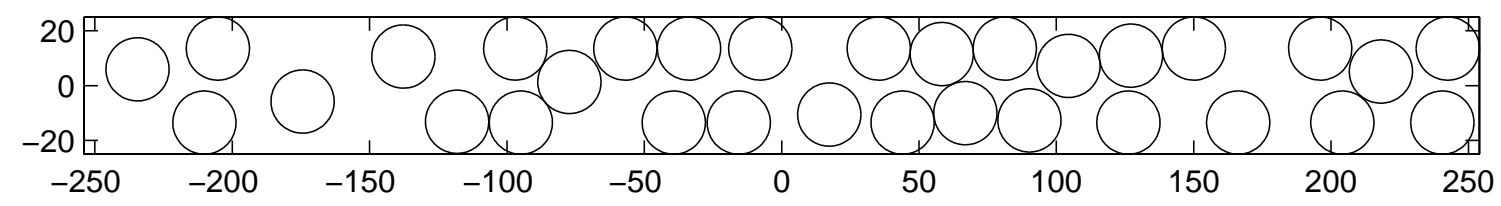

Figure 3.20: Random geometry, 31 particles particles of radius 11.5

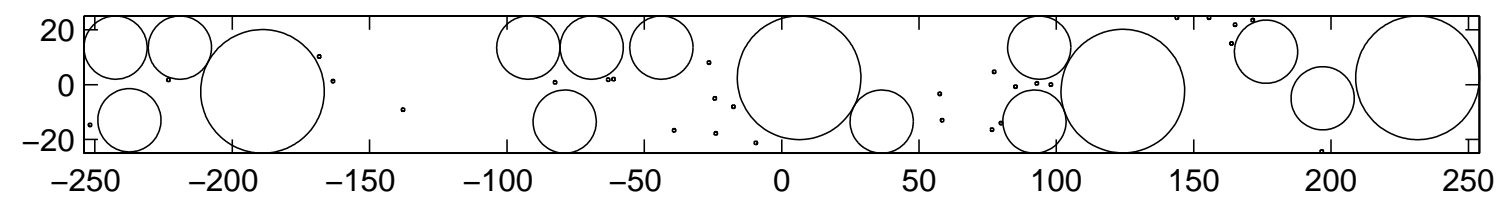

Figure 3.21: Random geometry, 44 particles particles of radius $22.5,11.5$, and 0.625 satisfying a 1:3:7 distribution

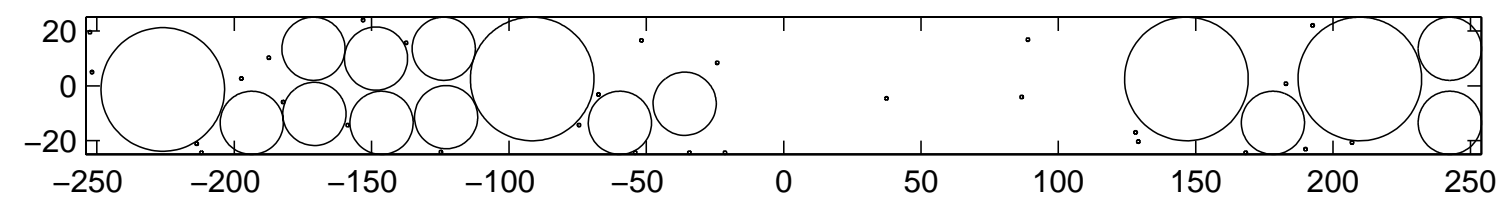

Figure 3.22: Random geometry, 44 particles particles of radius $22.5,11.5$, and 0.625 satisfying a 1:3:7 distribution

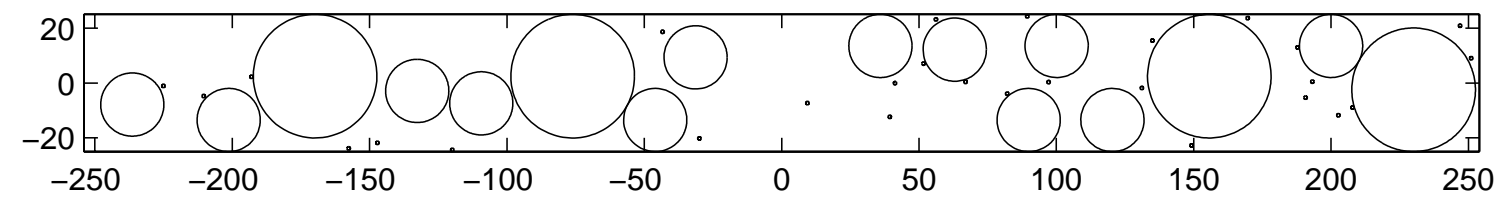

Figure 3.23: Random geometry, 44 particles particles of radius $22.5,11.5$, and 0.625 satisfying a 1:3:7 distribution 

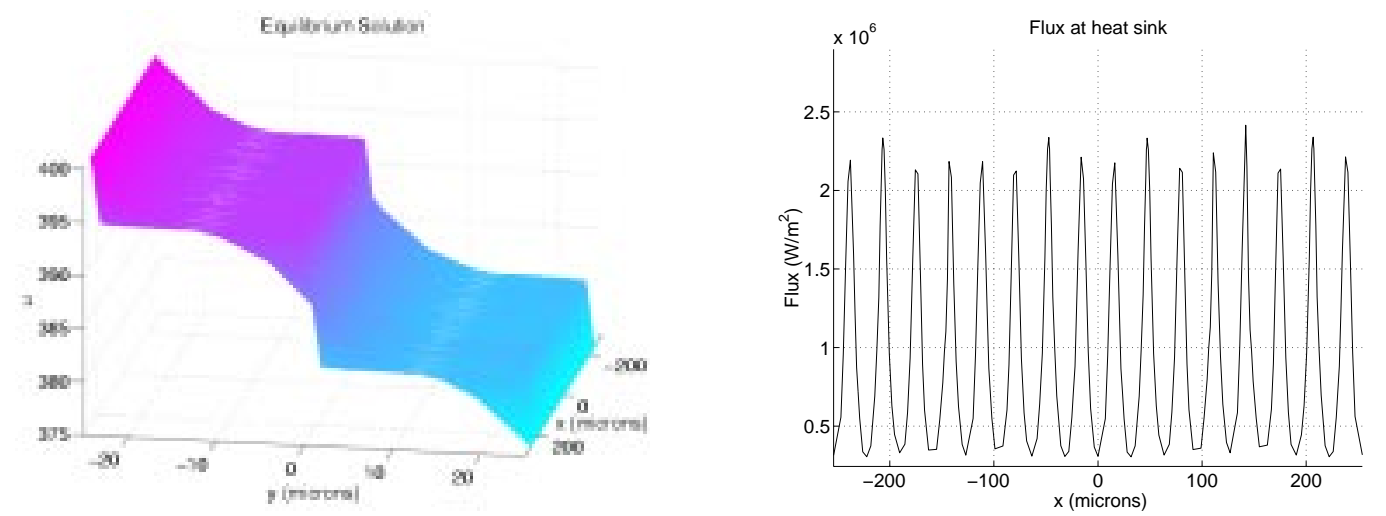

Figure 3.24: Solution (left) and flux at heat sink interface (right) using the uniform geometry with same sized particles in Figure 3.11
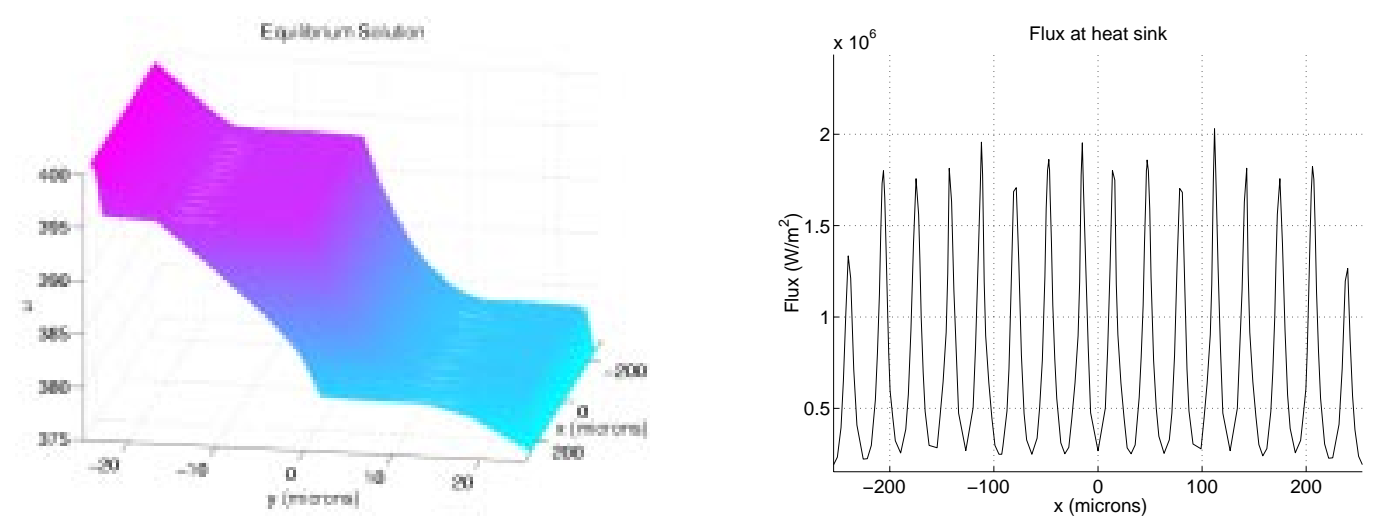

Figure 3.25: Solution (left) and flux at heat sink interface (right) using the shifted geometry with same sized particles in Figure 3.15 

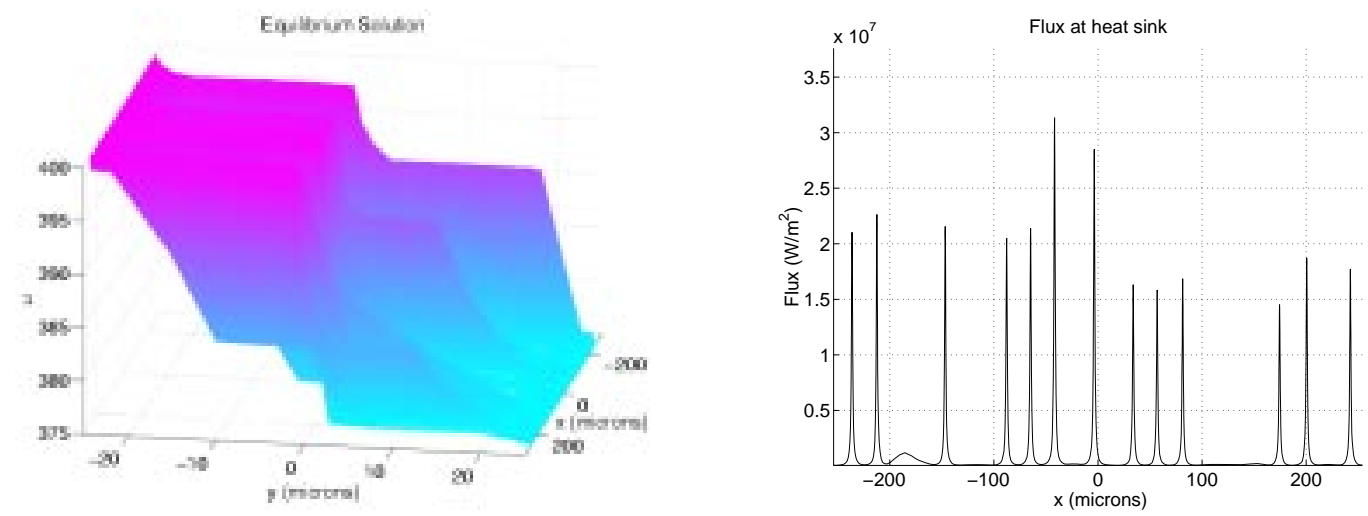

Figure 3.26: Solution (left) and flux at heat sink interface (right) using the random geometry with same sized particles in Figure 3.19
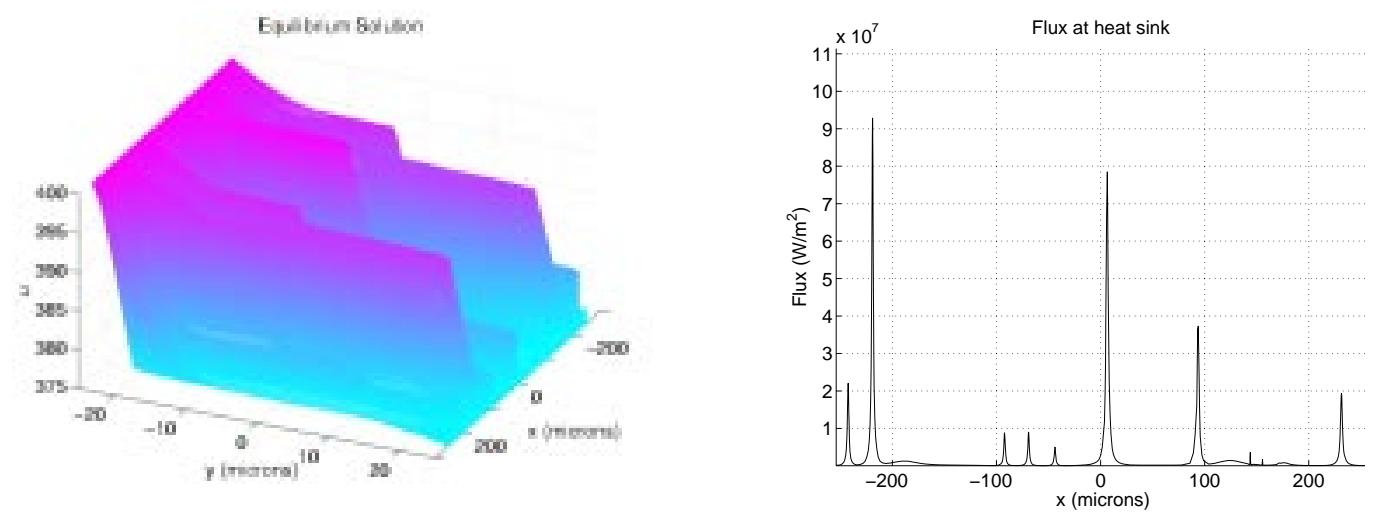

Figure 3.27: Solution (left) and flux at heat sink interface (right) using the random geometry with three different sized particles in Figure 3.21 
for each of the 14 geometries. In each norm, for the geometries with particles of the same size, the norm of the shifted geometry is less than the norm of the uniform geometry which is less than the norm of the random geometry. For the geometries with three different sized particles, the norm of the uniform geometry is less than the norm of the random geometry. Also, the geometries with a uniform arrangement of two different sized particles and three different sized particles have nearly the same norms, although in each norm the two sized particle geometry has slightly smaller norms than the three sized particle geometry. Since we expect the actual geometry of the composite to be more like the random geometries then either the uniform or shifted geometries, these examples suggest the uniform geometry should provide a lower bound for the flux (and presumably other quantities) for the actual composite geometry.

Based on these observations, it is clear that the particle geometry has an impact on the heat transfer through the composite. Using a uniform geometry in computational studies is advantageous for several reasons: the geometry is much easier and faster to generate, mesh generation is faster and the mesh requires fewer elements, and the computation time needed to solve over a uniform geometry is significantly less than the time for a random geometry. Thus, for our study in the following chapters, we will concentrate only on the uniform geometry. 


\begin{tabular}{|c|c|c|c|c|c|c|c|c|}
\hline $\begin{array}{c}\text { Figure } \\
\text { Number }\end{array}$ & $\begin{array}{c}\text { Geometry } \\
\text { Type }\end{array}$ & $\begin{array}{c}\text { Particle } \\
\text { Radii }\end{array}$ & $\begin{array}{c}\text { Particle } \\
\text { Dist. }\end{array}$ & $\begin{array}{c}\text { Number of } \\
\text { Particles }\end{array}$ & $\begin{array}{c}\text { Actual } \\
\text { \% Area }\end{array}$ & $\|F l u x\|_{1}$ & $\|F l u x\|_{2}$ & $\|F l u x\|_{\infty}$ \\
\hline \hline 3.10 & uniform & 22.5 & 1 & 8 & 50.09 & $4.052 \mathrm{e}+8$ & $2.103 \mathrm{e}+7$ & $1.890 \mathrm{e}+6$ \\
\hline 3.11 & uniform & 11.5 & 1 & 32 & 52.34 & $4.797 \mathrm{e}+8$ & $2.577 \mathrm{e}+7$ & $2.415 \mathrm{e}+6$ \\
\hline 3.12 & uniform & 6 & 1 & 112 & 49.87 & $6.704 \mathrm{e}+8$ & $4.117 \mathrm{e}+7$ & $4.739 \mathrm{e}+6$ \\
\hline 3.13 & uniform & $22.5: 11.5$ & $1: 4$ & 20 & 51.21 & $4.501 \mathrm{e}+8$ & $2.414 \mathrm{e}+7$ & $2.497 \mathrm{e}+6$ \\
\hline 3.14 & uniform & $22.5: 11.5: 0.625$ & $4: 16: 39$ & 59 & 51.41 & $4.495 \mathrm{e}+8$ & $2.426 \mathrm{e}+7$ & $2.430 \mathrm{e}+6$ \\
\hline 3.15 & shifted & 11.5 & 1 & 31 & 50.71 & $3.687 \mathrm{e}+8$ & $1.984 \mathrm{e}+7$ & $2.030 \mathrm{e}+6$ \\
\hline 3.16 & shifted & 6 & 1 & 110 & 48.98 & $3.343 \mathrm{e}+8$ & $2.105 \mathrm{e}+7$ & $2.732 \mathrm{e}+6$ \\
\hline 3.17 & random & 22.5 & 1 & 8 & 50.09 & $5.072 \mathrm{e}+8$ & $5.875 \mathrm{e}+7$ & $3.146 \mathrm{e}+6$ \\
\hline 3.18 & random & 22.5 & 1 & 8 & 50.09 & $7.577 \mathrm{e}+8$ & $1.464 \mathrm{e}+8$ & $1.105 \mathrm{e}+8$ \\
\hline 3.19 & random & 11.5 & 1 & 31 & 50.71 & $6.112 \mathrm{e}+8$ & $7.958 \mathrm{e}+7$ & $3.133 \mathrm{e}+7$ \\
\hline 3.20 & random & 11.5 & 1 & 31 & 50.71 & $7.240 \mathrm{e}+8$ & $1.074 \mathrm{e}+8$ & $5.503 \mathrm{e}+7$ \\
\hline 3.21 & random & $22.5: 11.5: 0.625$ & $1: 3: 7$ & 44 & 44.81 & $9.887 \mathrm{e}+8$ & $1.690 \mathrm{e}+8$ & $9.283 \mathrm{e}+7$ \\
\hline 3.22 & random & $22.5: 11.5: 0.625$ & $1: 3: 7$ & 44 & 44.81 & $7.581 \mathrm{e}+8$ & $1.165 \mathrm{e}+8$ & $4.748 \mathrm{e}+7$ \\
\hline 3.23 & random & $22.5: 11.5: 0.625$ & $1: 3: 7$ & 44 & 44.81 & $9.989 \mathrm{e}+8$ & $2.041 \mathrm{e}+8$ & $1.462 \mathrm{e}+8$ \\
\hline
\end{tabular}

Table 3.3: Measurements of the flux at the interface between composite and heat sink for different geometries 


\section{Chapter 4}

\section{Homogenization}

The oscillatory nature of the thermal conductivity makes it challenging to solve our problem without a tool like the PDE Toolbox (which is not available for $\Omega \in \mathbb{R}^{3}$ ). In this chapter we present an alternative approach for investigating thermal conductivity in the composite silicone using the mathematical theory of homogenization (which is available for $\Omega \in \mathbb{R}^{3}$ ). By concentrating on the microscopic structure of the composite silicone from Chapter 3 one can use homogenization theory to determine the "effective thermal conductivity" of the composite silicone, an "averaged value" for the thermal conductivity which is constant throughout the composite. See [2, 13, 23, 24, 30] for further information on homogenization theory. While we will not use homogenization theory further in our study, we include it here for completeness.

\subsection{Model with Periodic Microstructure}

Let $\Omega \subset \mathbb{R}^{2}$ or $\mathbb{R}^{3}$, depending on the desired dimension of the model, be a sample of the composite silicone with the periodic microstructure exhibited in Figure 4.1. It is possible to change the geometry of the cell, for example, we may have different sized particles or several particles in each cell, however it must be periodic in the cells for our considerations outlined here. We restrict our analysis here to the microstructure in Figure 4.1, but all results extend in a natural way to other periodic microstructures. 
We will assume each periodicity cell, $Y$, is the parallelogram defined by

$$
Y=\left[\frac{-c_{1}}{2}, \frac{c_{1}}{2}\right] \times \cdots \times\left[\frac{-c_{n}}{2}, \frac{c_{n}}{2}\right]
$$

for some positive constants $c_{1}, \cdots, c_{n}, n=2$ or $n=3$. We will assume the thermal conductivity $k$ is $Y$-periodic, i.e., $k(z)=k\left(z+c_{i} e_{i}\right)$ for $z=\left(z_{1}, \cdots, z_{n}\right) \in \Omega$ where $e_{1}, \cdots, e_{n}$ are the standard unit vectors in $\mathbb{R}^{n}$.

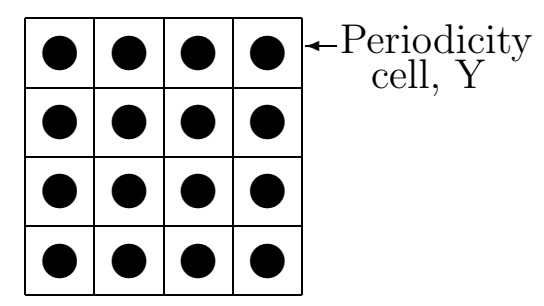

Figure 4.1: An example of a periodic microstructure in 2 dimensions

As in Chapter 3, since the composite silicone is locally isotropic, the thermal conductivity $k$ will have two different values:

$$
k(z)= \begin{cases}k_{s} & z \in \Omega_{s} \\ k_{p} & z \in \Omega_{p}\end{cases}
$$

We will assume we are looking at a steady state model, i.e., the temperature $u$ satisfies

$$
\nabla \cdot(k \nabla u)=0
$$

with appropriate boundary conditions; however all of our analysis in this chapter extends to the transient model (see, e.g., [2]).

Now choose $\epsilon$ so that $\epsilon$ is the length of the period of the sample. Define $k^{\epsilon}: \mathbb{R}^{n} \rightarrow$ $M^{n \times n}$ by

$$
k^{\epsilon}(z)=k\left(\frac{z}{\epsilon}\right)
$$

and note $k^{\epsilon}$ is $\epsilon Y$-periodic. Then in the periodicity cells if $u^{\epsilon}$ is defined by

$$
u^{\epsilon}(z)=u\left(\frac{z}{\epsilon}\right)
$$


it satisfies

$$
\nabla \cdot\left(k^{\epsilon} \nabla u^{\epsilon}\right)=0
$$

with the boundary conditions that enforce continuity across the cell interfaces. In the next section we present the basic ideas of homogenization in $n$-dimensions.

\subsection{Homogenization in $n$-Dimensions}

We will define a periodicity cell $Y \in \mathbb{R}^{n}$ by

$$
Y=\left[-\frac{c_{1}}{2}, \frac{c_{1}}{2}\right] \times \cdots \times\left[-\frac{c_{n}}{2}, \frac{c_{n}}{2}\right] .
$$

We will define $z \in \mathbb{R}^{n}$ as the point $z=\left(z_{1}, z_{2}, \cdots, z_{n}\right)$. We will assume $k(z)$ is the thermal conductivity for an n-dimensional system and so $k(z)$ is a diagonal matrix.

We define $S_{\#}$ to be the set of $Y$-periodic functions $a: \mathbb{R}^{n} \rightarrow M^{n \times n}$ such that $a(z)=\left[a_{i j}(z)\right], i, j=1, \cdots, n$ which satisfy the following conditions:

i) $a_{i j} \in L^{\infty}$ for $i, j=1, \cdots, n$

ii) $a_{i j}=a_{j i}$ for $i, j=1, \cdots, n$

iii) there exists a constant $\alpha \geq 0$ so that $(a(z) \zeta, \zeta) \geq \alpha|\zeta|^{2}$.

If $k \in S_{\#}$, then as $\epsilon \rightarrow 0$

$$
\begin{array}{ll}
u^{\epsilon} \rightarrow u^{0} & \text { weakly in } L^{2}\left(\Omega, \mathbb{R}^{n}\right) \\
k\left(\frac{z}{\epsilon}\right) u^{\epsilon} \rightarrow \bar{k} u^{0} & \text { weakly in } L^{2}\left(\Omega, \mathbb{R}^{n}\right)
\end{array}
$$

where $u^{0}$ solves the homogenized problem

$$
\nabla \cdot\left(\bar{k} \nabla u^{0}\right)=0
$$

$\bar{k}$ is given in (4.1) below, and $u^{\epsilon}$ solves

$$
\nabla \cdot\left(k\left(\frac{z}{\epsilon}\right) \nabla u^{\epsilon}\right)=0 .
$$

It is easily verified that $k \in S_{\#}$ if the material is isotropic. Thus we know from [13] that the homogenized coefficient, $\bar{k}$ is given by

$$
(\bar{k})_{i j}=\frac{1}{|Y|} \int_{Y}\left(k_{i j}(z)+\sum_{m=1}^{n} k_{i m}(z) \frac{\partial w_{j}}{\partial z_{m}}(z)\right) d z
$$


where $w_{j}$ is the unique solution satisfying

$$
\left\{\begin{array}{l}
\nabla \cdot\left(k(z)\left(e_{j}+\nabla w_{j}(z)\right)\right)=0 \quad \text { for all } z \in Y \\
w_{j} \in H_{\#}^{1}(Y)
\end{array}\right.
$$

where $H^{1}(Y)=\left\{f \in L^{2}(Y): \nabla f \in L^{2}(Y)\right\}$ and $H_{\#}^{1}(Y)$ is the set of all periodic functions that are in $H^{1}(Y)$.

Since $k(z)$ is diagonal, we can rewrite (4.1) as

$$
(\bar{k})_{i j}=\frac{1}{|Y|} \int_{Y} k_{i i}(z) \frac{\partial w_{j}}{\partial z_{i}}(z) d z
$$

if $i \neq j$ and

$$
(\bar{k})_{i i}=\frac{1}{|Y|} \int_{Y} k_{i i}(z)\left(1+\frac{\partial w_{i}}{\partial z_{i}}(z)\right) d z
$$

for $i, j=1, \cdots n$.

If we assume the composite silicone is isotropic and $k(z)$ is even in $z_{j}$ for all $j$ we can show that $\bar{k}$ is a diagonal matrix and that $w_{j}$ is even in $z_{i}$ for $i \neq j$ and odd in $z_{j}$. To simplify notation, let $k_{i i}(z)=k(z)$ for all $i$.

First note

$$
\begin{gathered}
\nabla \cdot\left(k(z)\left(e_{j}+\nabla w_{j}\right)\right)=\nabla \cdot\left(k(z)\left[\begin{array}{c}
\frac{\partial w_{j}}{\partial z_{1}}(z) \\
\vdots \\
1+\frac{\partial w_{j}}{\partial z_{j}}(z) \\
\vdots \\
\frac{\partial w_{j}}{\partial z_{n}}(z)
\end{array}\right]\right) \\
=\nabla \cdot\left(\left[\begin{array}{c}
k(z) \frac{\partial w_{j}}{\partial z_{1}}(z) \\
\vdots \\
k(z)\left(1+\frac{\partial w_{j}}{\partial z_{j}}(z)\right) \\
\vdots \\
k(z) \frac{\partial w_{j}}{\partial z_{n}}(z)
\end{array}\right]\right)
\end{gathered}
$$


Chapter 4. Homogenization

$$
\begin{aligned}
=\sum_{i \neq j} & \left(\frac{\partial k}{\partial z_{i}}(z) \frac{\partial w_{j}}{\partial z_{i}}(z)+k(z) \frac{\partial^{2} w_{j}}{\partial z_{i}^{2}}(z)\right) \\
& +\frac{\partial k}{\partial z_{j}}(z)\left(1+\frac{\partial w_{j}}{\partial z_{j}}(z)\right)+k(z) \frac{\partial^{2} w_{j}}{\partial z_{j}^{2}}(z)
\end{aligned}
$$

for all $y \in Y$. Let $\tilde{x}_{j}=\left[x_{1}, \cdots, x_{n}\right]$ and let $\hat{x}_{j}=\left[x_{1}, \cdots,-x_{j}, \cdots, x_{n}\right]$ for $j=$ $1, \cdots, n, \tilde{x}_{j}, \hat{x}_{j} \in Y$. Since $k$ is even in $z_{j}$ for all $j, k\left(\tilde{x}_{j}\right)=k\left(\hat{x}_{j}\right)$. Let $\tilde{w}_{j}(z) \in H_{\#}^{1}(Y)$ and let $-\tilde{w}_{j}\left(\hat{x}_{j}\right)=w_{j}\left(\tilde{x}_{j}\right)$. Then

$$
\begin{aligned}
0=\sum_{i \neq j}( & \left.\frac{\partial k}{\partial z_{i}}\left(\tilde{x}_{j}\right) \frac{\partial w_{j}}{\partial z_{i}}\left(\tilde{x}_{j}\right)+k\left(\tilde{x}_{j}\right) \frac{\partial^{2} w_{j}}{\partial z_{i}^{2}}\left(\tilde{x}_{j}\right)\right) \\
& +\frac{\partial k}{\partial z_{j}}\left(\tilde{x}_{j}\right)\left(1+\frac{\partial w_{j}}{\partial z_{j}}\left(\tilde{x}_{j}\right)\right)+k\left(\tilde{x}_{j}\right) \frac{\partial^{2} w_{j}}{\partial z_{j}^{2}}\left(\tilde{x}_{j}\right) \\
=\sum_{i \neq j}( & \left.\frac{\partial k}{\partial z_{i}}\left(\tilde{x}_{j}\right)\left(-\frac{\partial \tilde{w}_{j}}{\partial z_{i}}\left(\hat{x}_{j}\right)\right)+k\left(\tilde{x}_{j}\right)\left(-\frac{\partial^{2} \tilde{w}_{j}}{\partial z_{i}^{2}}\left(\hat{x}_{j}\right)\right)\right) \\
& +\frac{\partial k}{\partial z_{j}}\left(\tilde{x}_{j}\right)\left(1+\frac{\partial \tilde{w}_{j}}{\partial z_{j}}\left(\hat{x}_{j}\right)\right)+k\left(\tilde{x}_{j}\right)\left(-\frac{\partial^{2} \tilde{w}_{j}}{\partial z_{j}^{2}}\left(\hat{x}_{j}\right)\right) \\
=\sum_{i \neq j} & \left(-\frac{\partial k}{\partial z_{i}}\left(\hat{x}_{j}\right) \frac{\partial \tilde{w}_{j}}{\partial z_{i}}\left(\hat{x}_{j}\right)-k\left(\hat{x}_{j}\right) \frac{\partial^{2} \tilde{w}_{j}}{\partial z_{i}^{2}}\left(\hat{x}_{j}\right)\right) \\
& -\frac{\partial k}{\partial z_{j}}\left(\hat{x}_{j}\right)\left(1+\frac{\partial \tilde{w}_{j}}{\partial z_{j}}\left(\hat{x}_{j}\right)\right)-k\left(\hat{x}_{j}\right) \frac{\partial^{2} \tilde{w}_{j}}{\partial z_{j}^{2}}\left(\hat{x}_{j}\right) \\
=-\{ & \sum_{i \neq j}\left(\frac{\partial k}{\partial z_{i}}\left(\hat{x}_{j}\right) \frac{\partial \tilde{w}_{j}}{\partial z_{i}}\left(\hat{x}_{j}\right)+k\left(\hat{x}_{j}\right) \frac{\partial^{2} \tilde{w}_{j}}{\partial z_{i}^{2}}\left(\hat{x}_{j}\right)\right) \\
& \left.+\frac{\partial k}{\partial z_{j}}\left(\hat{x}_{j}\right)\left(1+\frac{\partial \tilde{w}_{j}}{\partial z_{j}}\left(\hat{x}_{j}\right)\right)+k\left(\hat{x}_{j}\right) \frac{\partial^{2} \tilde{w}_{j}}{\partial z_{j}^{2}}\left(\hat{x}_{j}\right)\right\} .
\end{aligned}
$$

Since $w_{j}(z)$ is the unique solution to $(4.2)$ for all $z \in Y, \tilde{w}_{j}\left(\hat{x}_{j}\right)=w_{j}\left(\hat{x}_{j}\right)$, and so $w_{j}\left(\hat{x}_{j}\right)=-w_{j}\left(\tilde{x}_{j}\right)$. Thus $w_{j}(z)$ is odd in $z_{j}$.

Now suppose $w_{j}\left(\tilde{x}_{m}\right)=\tilde{w}_{j}\left(\hat{x}_{m}\right)$ for $j \neq m$. Then

$$
0=\sum_{i \neq j}\left(\frac{\partial k}{\partial z_{i}}\left(\tilde{x}_{m}\right) \frac{\partial w_{j}}{\partial z_{i}}\left(\tilde{x}_{m}\right)+k\left(\tilde{x}_{m}\right) \frac{\partial^{2} w_{j}}{\partial z_{i}^{2}}\left(\tilde{x}_{m}\right)\right)
$$




$$
\begin{aligned}
&+\frac{\partial k}{\partial z_{j}}\left(\tilde{x}_{m}\right)\left(1+\frac{\partial w_{j}}{\partial z_{j}}\left(\tilde{x}_{m}\right)\right)+k\left(\tilde{x}_{m}\right) \frac{\partial^{2} w_{j}}{\partial z_{j}^{2}}\left(\tilde{x}_{m}\right) \\
&=\sum_{i \neq j \neq m}\left(\frac{\partial k}{\partial z_{i}}\left(\tilde{x}_{m}\right) \frac{\partial \tilde{w}_{j}}{\partial z_{i}}\left(\hat{x}_{m}\right)+k\left(\tilde{x}_{m}\right) \frac{\partial^{2} \tilde{w}_{j}}{\partial z_{i}^{2}}\left(\hat{x}_{m}\right)\right) \\
&+\frac{\partial k}{\partial z_{m}}\left(\tilde{x}_{m}\right)\left(-\frac{\partial \tilde{w}_{j}}{\partial z_{m}}\left(\hat{x}_{m}\right)\right)+k\left(\tilde{x}_{m}\right) \frac{\partial^{2} \tilde{w}_{j}}{\partial z_{m}^{2}}\left(\hat{x}_{m}\right) \\
&+\frac{\partial k}{\partial z_{j}}\left(\tilde{x}_{m}\right)\left(1+\frac{\partial \tilde{w}_{j}}{\partial z_{j}}\left(\hat{x}_{m}\right)\right)+k\left(\tilde{x}_{m}\right) \frac{\partial^{2} \tilde{w}_{j}}{\partial z_{j}^{2}}\left(\hat{x}_{m}\right) \\
&=\sum_{i \neq j \neq m}\left(\frac{\partial k}{\partial z_{i}}\left(\hat{x}_{m}\right) \frac{\partial \tilde{w}_{j}}{\partial z_{i}}\left(\hat{x}_{m}\right)+k\left(\hat{x}_{m}\right) \frac{\partial^{2} \tilde{w}_{j}}{\partial z_{i}^{2}}\left(\hat{x}_{m}\right)\right) \\
&+\frac{\partial k}{\partial z_{m}}\left(\hat{x}_{m}\right) \frac{\partial \tilde{w}_{j}}{\partial z_{m}}\left(\hat{x}_{m}\right)+k\left(\hat{x}_{m}\right) \frac{\partial^{2} \tilde{w}_{j}}{\partial z_{m}^{2}}\left(\hat{x}_{m}\right) \\
&+\frac{\partial k}{\partial z_{j}}\left(\hat{x}_{m}\right)\left(1+\frac{\partial \tilde{w}_{j}}{\partial z_{j}}\left(\hat{x}_{m}\right)\right)+k\left(\hat{x}_{m}\right) \frac{\partial^{2} \tilde{w}_{j}}{\partial z_{j}^{2}}\left(\hat{x}_{m}\right) \\
&+\frac{\partial k}{\partial z_{j}}\left(\hat{x}_{m}\right)\left(1+\frac{\partial \tilde{w}_{j}}{\partial z_{j}}\left(\hat{x}_{m}\right)\right)+k\left(\hat{x}_{m}\right) \frac{\partial^{2} \tilde{w}_{j}}{\partial z_{j}^{2}}\left(\hat{x}_{m}\right) \\
&=\sum_{i \neq j}\left(\frac{\partial k}{\partial z_{i}}\left(\hat{x}_{m}\right) \frac{\partial \tilde{w}_{j}}{\partial z_{i}}\left(\hat{x}_{m}\right)+k\left(\hat{x}_{m}\right) \frac{\partial^{2} \tilde{w}_{j}}{\partial z_{i}^{2}}\left(\hat{x}_{m}\right)\right)
\end{aligned}
$$

Since $w_{j}(z)$ is the unique solution to $(4.2)$ for all $z \in Y, \tilde{w}_{j}\left(\hat{x}_{m}\right)=w_{j}\left(\hat{x}_{m}\right)$, and so $w_{j}\left(\hat{x}_{m}\right)=w_{j}\left(\tilde{x}_{m}\right)$. Thus $w_{j}(z)$ is even in $z_{m}$ for $j \neq m$.

So

$$
(\bar{k})_{i j}=\frac{1}{|Y|} \int_{Y} k_{i i}(z) \frac{\partial w_{j}}{\partial z_{i}}(z) d z=0
$$

for $i \neq j$ and

$$
(\bar{k})_{i i}=\frac{2^{n}}{|Y|} \int_{\tilde{Y}} k_{i i}(z)\left(1+\frac{\partial w_{i}}{\partial z_{i}}(z)\right) d z
$$

where $\tilde{Y}=\left[0, \frac{c_{1}}{2}\right] \times \cdots \times\left[0, \frac{c_{n}}{2}\right]$. Thus $\bar{k}$ is a diagonal matrix with diagonal entries given by (4.3). 
Since it is only necessary to know $w_{j}$ on $\tilde{Y}$, we would like to be able to rewrite (4.2) so that we only need to solve for $w_{j}$ on $\tilde{Y}$ rather than on $Y$. We need to derive boundary conditions that will ensure $w_{j} \in H_{\#}^{1}(Y)$ and that $w_{j}$ is even in $z_{i}$ for $i \neq j$ and odd in $z_{j}$.

Define $\bar{x}(j, a)=\left[x_{1}, \ldots, x_{j-1}, a, x_{j+1}, \ldots, x_{n}\right]$. Since $w_{j} \in H_{\#}^{1}(Y)$, we know

$$
w_{j}\left(\bar{x}\left(j, \frac{c_{j}}{2}\right)\right)=w_{j}\left(\bar{x}\left(j,-\frac{c_{j}}{2}\right)\right)
$$

But $w_{j}$ is odd in $z_{j}$, so

$$
w_{j}\left(\bar{x}\left(j, \frac{c_{j}}{2}\right)\right)=-w_{j}\left(\bar{x}\left(j,-\frac{c_{j}}{2}\right)\right) .
$$

Thus $w_{j}\left(\bar{x}\left(j, \frac{c_{j}}{2}\right)\right)=0$, and so $w_{j}=0$ when $z_{j}=\frac{c_{j}}{2}$. Again since $w_{j}$ is odd in $z_{j}$,

$$
w_{j}(\bar{x}(j, \delta))=-w_{j}(\bar{x}(j,-\delta))
$$

for all $\delta \in\left[-\frac{c_{j}}{2}, \frac{c_{j}}{2}\right]$. If we let $\delta \rightarrow 0$, we see

$$
w_{j}(\bar{x}(j, 0))=-w_{j}(\bar{x}(j, 0))
$$

so $w_{j}=0$ when $z_{j}=0$. Since $w_{j}$ is even in $z_{i}$ for $i \neq j$, it is not necessary to put any restrictions on $w_{j}$ when $z_{i}=0, \frac{c_{i}}{2}$.

Now note that if $\tilde{n}$ is the unit outward normal to the face of $\tilde{Y}$ where $z_{i}=\frac{c_{i}}{2}$, $\tilde{n}=e_{i}$, where $e_{i}$ is the standard unit vector. So on $z_{i}=\frac{c_{i}}{2}$,

$$
\frac{\partial w_{j}}{\partial \tilde{n}}=\nabla w_{j} \cdot \tilde{n}=\frac{\partial w_{j}}{\partial z_{i}}
$$

Since $w_{j} \in H_{\#}^{1}(Y)$,

$$
\frac{\partial w_{j}}{\partial z_{i}}\left(\bar{x}\left(i, \frac{c_{i}}{2}\right)\right)=\frac{\partial w_{j}}{\partial z_{i}}\left(\bar{x}\left(i,-\frac{c_{i}}{2}\right)\right) .
$$

But, $w_{j}$ is even in $z_{i}$, so

$$
\frac{\partial w_{j}}{\partial z_{i}}\left(\bar{x}\left(i, \frac{c_{i}}{2}\right)\right)=-\frac{\partial w_{j}}{\partial z_{i}}\left(\bar{x}\left(i,-\frac{c_{i}}{2}\right)\right) .
$$


Thus $\frac{\partial w_{j}}{\partial z_{i}}\left(\bar{x}\left(i, \frac{c_{i}}{2}\right)\right)=0$ and so $\frac{\partial w_{j}}{\partial \tilde{n}}=0$ on $z_{i}=\frac{c_{i}}{2}$. Again using the fact $w_{j}$ is even in $z_{i}$

$$
\frac{\partial w_{j}}{\partial z_{i}}(\bar{x}(i, \delta))=-\frac{\partial w_{j}}{\partial z_{i}}(\bar{x}(i,-\delta))
$$

Letting $\delta \rightarrow 0$ we see

$$
\frac{\partial w_{j}}{\partial z_{i}}(\bar{x}(i, 0))=-\frac{\partial w_{j}}{\partial z_{i}}(\bar{x}(j, 0)) .
$$

The unit outward normal to $z_{i}=0$ is $\tilde{n}=-e_{i}$, so $\frac{\partial w_{j}}{\partial \tilde{n}}=0$ when $z_{i}=0$. Since $w_{j}$ is odd in $z_{j}$, it is not necessary to put any restrictions on $\frac{\partial w_{j}}{\partial \tilde{n}}$ when $z_{j}=0, \frac{c_{i}}{2}$.

If we define

$$
Y_{1}^{j}=\left\{z \in \tilde{Y}: z_{j}=0, \frac{c_{j}}{2}\right\}
$$

and

$$
Y_{2}^{j}=\partial \tilde{Y}-Y_{1}^{j}
$$

we see that it is sufficient to find the unique solution $w_{j}$ satisfying

$$
\begin{cases}\nabla \cdot\left(k(z)\left(e_{j}+\nabla w_{j}(z)\right)\right)=0 & \text { for all } z \in \tilde{Y} \\ w_{j}=0 & \text { on } Y_{1}^{j} \\ \frac{\partial w_{j}}{\partial \tilde{n}}=0 & \text { on } Y_{2}^{j}\end{cases}
$$

where $\tilde{n}$ is the unit outward normal on $Y_{2}^{j}$ and the solution $w_{j}$ will still satisfy (4.2).

\subsection{Homogenization in Two Dimensions}

We will concentrate here on homogenization in the two dimensional problem; our analysis easily extends to the three dimensional case. We start with the steady state heat equation

$$
\nabla \cdot(k(z) \nabla u(z))=0
$$

where $z \in \mathbb{R}^{2}, u(z)$ is the temperature at $z$, and $k(z)$ is the thermal conductivity at $z$. 
We will assume each periodicity cell is $Y=\left[-\frac{c_{1}}{2}, \frac{c_{1}}{2}\right] \times\left[-\frac{c_{2}}{2}, \frac{c_{2}}{2}\right]$ and that the filler particles are uniformly shaped circles of radius $r\left(r<c_{1}\right.$ and $\left.r<c_{2}\right)$ centered in each cell. We will also assume the material is locally isotropic, so for each $z \in Y$,

$$
k(z)=\left\{\begin{array}{ll}
k_{s} & |z|>r \\
k_{p} & |z| \leq r
\end{array} .\right.
$$

If $\epsilon$ is the length of the period of the samples, using the same conventions as in Section 4.1, we can rewrite our problem on the microscopic level as

$$
\left\{\begin{array}{l}
\nabla \cdot\left(k\left(\frac{z}{\epsilon}\right) \nabla u^{\epsilon}(z)\right)=0 \\
\left.u^{\epsilon}\right|_{\xi_{1}}=\left.u^{\epsilon}\right|_{\xi_{3}} \\
\left.u^{\epsilon}\right|_{\xi_{2}}=\left.u^{\epsilon}\right|_{\xi_{4}}
\end{array}\right.
$$

where $\xi_{1}, \xi_{2}, \xi_{3}$, and $\xi_{4}$ are defined as in Figure 4.2. The boundary conditions are chosen so that the solution is continuous across cell interfaces.

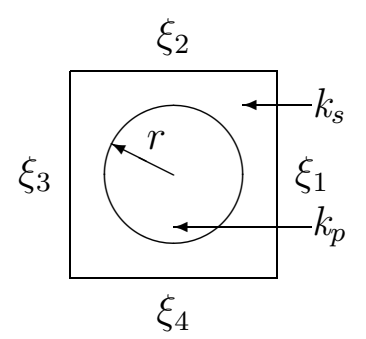

Figure 4.2: Periodicity cell, $Y$

Then we can define $\tilde{Y}=\left[0, \frac{c_{1}}{2}\right] \times\left[0, \frac{c_{2}}{2}\right]$ and our homogenized coefficients are:

$$
\begin{aligned}
& \bar{k}_{11}=\frac{2^{2}}{|Y|} \int_{\tilde{Y}} k_{11}(z)\left(1+\frac{\partial w_{1}}{\partial z_{1}}(z)\right) d z \\
& \bar{k}_{22}=\frac{2^{2}}{|Y|} \int_{\tilde{Y}} k_{22}(z)\left(1+\frac{\partial w_{2}}{\partial z_{2}}(z)\right) d z
\end{aligned}
$$




$$
\bar{k}_{12}=\bar{k}_{21}=0
$$

where $w_{j}$ solves

$$
\begin{cases}\nabla \cdot\left(k(z)\left(e_{j}+\nabla w_{j}(z)\right)\right)=0 & \text { for all } z \in \tilde{Y} \\ w_{j}=0 & \text { on } Y_{1}^{j} \\ \frac{\partial w_{j}}{\partial n}=0 & \text { on } Y_{2}^{j}\end{cases}
$$

with $Y_{1}^{j}=\left\{z \in \tilde{Y}: z_{j}=0, \frac{c_{j}}{2}\right\}$ and $Y_{2}^{j}=\partial \tilde{Y}-Y_{1}^{j}$ for $j=1,2$.

\subsection{Using Matlab to Find the Homogenized Coef- ficient}

Matlab's PDE Toolbox cannot solve (4.7) directly, it can only solve parabolic systems of the form $d \frac{\partial u}{\partial t}-\nabla \cdot(c \nabla u)+a u=f$. Instead we solve a slightly different problem and from the solution to this problem compute the solution to (4.7). If we use Matlab to solve the following system,

$$
\left\{\begin{array}{l}
\nabla \cdot\left(k(z) \nabla u_{1}(z)\right)=0 \quad \text { for all } z \in \tilde{Y} \\
\left.u_{1}\right|_{\hat{\xi}_{1}}=\frac{c_{1}}{2} \\
\left.u_{1}\right|_{\hat{\xi}_{2}}=0 \\
\left.\frac{\partial u_{1}}{\partial n}\right|_{\hat{\xi}_{3}}=0 \\
\left.\frac{\partial u_{1}}{\partial n}\right|_{\hat{\xi}_{4}}=0
\end{array} .\right.
$$

and note that if we define $u_{1}(z)=x+w_{1}(z)$, this is equivalent to solving (4.7) for $j=1$ when

$$
\begin{aligned}
& \hat{\xi}_{1}=\left\{z=(x, y) \in \tilde{Y}: x=\frac{c_{1}}{2}\right\} \\
& \hat{\xi}_{2}=\left\{z=(x, y) \in \tilde{Y}: y=\frac{c_{2}}{2}\right\}
\end{aligned}
$$




$$
\begin{aligned}
& \hat{\xi}_{3}=\{z=(x, y) \in \tilde{Y}: x=0\} \quad \text { and } \\
& \hat{\xi}_{4}=\{z=(x, y) \in \tilde{Y}: y=0\} .
\end{aligned}
$$

Similarly, having Matlab solve

$$
\left\{\begin{array}{l}
\nabla \cdot\left(k(z) \nabla u_{2}(z)\right)=0 \quad \text { for all } z \in \tilde{Y} \\
\left.u_{2}\right|_{\hat{\xi}_{2}}=\frac{c_{2}}{2} \\
\left.u_{2}\right|_{\hat{\xi}_{4}}=0 \\
\left.\frac{\partial u_{2}}{\partial n}\right|_{\hat{\xi}_{1}}=0 \\
\left.\frac{\partial u_{2}}{\partial n}\right|_{\hat{\xi}_{3}}=0
\end{array}\right.
$$

is equivalent to solving (4.7) for $j=2$ where $u_{2}(z)=y+w_{2}(z)$.

Once Matlab solves for $u_{j}, w_{j}$ is computed using the above relations. Then $k_{j j}$ is computed numerically. There are two sources of error in this computation that should be noted. First the gradient of $w_{j}$ is computed. Here Matlab computes the gradient at the center of each triangle, and so the gradient must be interpolated back to the nodes. Second, in order to numerically integrate to find $k_{j j}$ the integrand must be interpolated to the points of integration used by Matlab's numerical integration routine. The interpolation routine uses bilinear interpolation. The integration is carried out with a double quadrature using an adaptive recursive Newton-Cotes 8 panel rule.

In our initial study of the heat transfer problem we used the PDE Toolbox to compute the homogenized coefficients. From our initial computations, it appeared we were not using a sufficiently fine microstructure. However, after becoming more familiar with the PDE Toolbox we discovered it was capable of directly solving the oscillatory problem.

For the remainder of our study of the heat transfer problem we use Matlab's PDE Toolbox directly on the problem with oscillatory coefficients. One distinct advantage of the PDE Toolbox is that it is easy to select any of the geometries presented in Section 3.4. In order to use homogenization on the random geometries we would 
need to use homogenization theory for random media. See, for example, [8, 30] for homogenization theory for random media. One advantage of homogenization theory is that it accounts for the variability in the composite silicone. However, in Chapter 6 , we present an approach to parameter estimation that allows us to represent the variability in our samples. 


\section{Chapter 5}

\section{Well-Posedness Results}

\subsection{Problem in Sesquilinear Form}

In this chapter we study theoretical issues relating to our two dimensional model from Chapter 3. We present a class of abstract parabolic equations and show this class of equations is well-posed. Furthermore, we show that our two dimensional heat transfer model fits this class of equations. These results guarantee the existence and uniqueness of weak solutions to our model as well as continuous dependence on the initial data, forcing function and model parameters.

\subsubsection{Preliminaries}

We start with the two dimensional model from Section 3.2

$$
\begin{cases}g(z) \dot{u}(t, z)=\nabla \cdot(k(z) \nabla u(t, z)) & \\ \left.k(z) \frac{\partial u}{\partial n}(t, z)\right|_{\gamma_{4}}=S_{0}(t) & \text { (source) } \\ \left.k(z) \frac{\partial u}{\partial n}(t, z)\right|_{\gamma_{2}}=\left.h\left(T_{\infty}-u(t, z)\right)\right|_{\gamma_{2}} & (\text { sink }) \\ \left.k(z) \frac{\partial u}{\partial n}(t, z)\right|_{\gamma_{1}}=0 & \\ \left.k(z) \frac{\partial u}{\partial n}(t, z)\right|_{\gamma_{3}}=0 & \\ u(0, z)=\Upsilon(z) & \end{cases}
$$


where for simplicity of notation we define $g(z)=\rho(z) c_{p}(z)$. We assume there exists constants $R_{L}$ and $R_{U}$ such that

$$
0<R_{L} \leq g(z) \leq R_{U}<\infty
$$

and constants $K_{L}$ and $K_{U}$ such that

$$
0<K_{L} \leq k(z) \leq K_{U}<\infty
$$

for all $z \in \Omega$. Furthermore we assume $h$ is a finite, positive constant.

Define $H=L^{2}(\Omega)$ with the usual $L^{2}$ inner product, $\langle\cdot, \cdot\rangle_{L^{2}}$, and define $\mathcal{H}=L^{2}(\Omega)$ with the weighted inner product $\langle\cdot, \cdot\rangle_{\mathcal{H}}=\langle g \cdot, \cdot\rangle_{L^{2}}$. Note the norms generated by the $\mathcal{H}$-inner product and the $L^{2}$-inner product are equivalent. Let $V=H^{1}(\Omega)$ with inner product

$$
\langle\phi, \psi\rangle_{V}=\langle\nabla \phi, \nabla \psi\rangle_{L^{2}}+\langle\phi, \psi\rangle_{L^{2}}
$$

for $\phi, \psi \in V$ and let $\mathcal{V}=H^{1}(\Omega)$ with inner product

$$
\langle\phi, \psi\rangle_{\mathcal{V}}=\langle\nabla \phi, \nabla \psi\rangle_{\mathcal{H}}+\langle\phi, \psi\rangle_{L^{2}}
$$

for $\phi, \psi \in \mathcal{V}$. We will want to use the following equivalent representation of the inner product in $\mathcal{V}$ :

$$
\langle\phi, \psi\rangle_{\mathcal{V}} \cong\langle\nabla \phi, \nabla \psi\rangle_{\mathcal{H}}+\int_{\gamma_{2}}\left(\operatorname{Tr}_{2} \phi\right)(z)\left(\operatorname{Tr}_{2} \psi\right)(z) d S_{2}
$$

where $\operatorname{Tr}_{j}: H^{1}(\Omega) \rightarrow H^{\frac{1}{2}}\left(\gamma_{j}\right)$ is the continuous trace operator mapping $f \in H^{1}(\Omega) \rightarrow$ $H^{\frac{1}{2}}\left(\gamma_{j}\right) \subset H^{0}\left(\gamma_{j}\right)=L^{2}\left(\gamma_{j}\right)$, on $\gamma_{j}, j=1,2,3,4$, with $\left(\operatorname{Tr}_{j} f\right)(z)=\left.f(z)\right|_{\gamma_{j}}$. The following theorem from Maz'ja [22, p. 27] shows the norms generated by these inner products are equivalent:

Theorem 5.1 (Maz'ja) Let $\Omega$ be a bounded domain such that $L_{p}^{\ell}(\Omega) \subset L_{p}(\Omega)$. Let $\mathcal{F}(u)$ be a continuous functional in $W_{p}^{\ell}(\Omega), \mathcal{F}\left(\Pi_{\ell-1}\right) \neq 0$ for any nonzero polynomial $\Pi_{\ell-1}$ of degree not higher than $\ell-1$. Then the norm

$$
\left\|\nabla_{\ell} u\right\|_{L_{p}(\Omega)}+\mathcal{F}(u)
$$

is equivalent to the norm in $W_{p}^{\ell}(\Omega)$. 
Here $L_{p}^{\ell}(\Omega)$ is the space of distributions on $\Omega$ with derivatives of order $\ell$ in $L_{p}(\Omega)$, and $W_{p}^{\ell}(\Omega)=L_{p}^{\ell}(\Omega) \cap L_{p}(\Omega)$. Also $\nabla_{\ell}=\left\{D^{\alpha}\right\}$, where $|\alpha|=\ell$ for $\alpha$ a multi-index $\left(\alpha_{1}, \cdots, \alpha_{n}\right)$ with $D^{\alpha}=D_{x_{1}}^{\alpha_{1}} \cdots D_{x_{n}}^{\alpha_{n}}$. Note for our problem $W_{p}^{\ell}(\Omega)=W_{2}^{1}(\Omega)=$ $H^{1}(\Omega)$, i.e., $p=2$ and $\ell=1$. If we define

$$
\mathcal{F}(u)=\int_{\Gamma}|\operatorname{tr}(u)|^{2} d x
$$

where $\Gamma \subset \partial \Omega$ and $t r$ is the continuous trace operator mapping $u \in H^{1}(\Omega) \rightarrow$ $H^{\frac{1}{2}}(\partial \Omega) \subset H^{0}(\partial \Omega)=L^{2}(\partial \Omega)$, then we have

$$
\begin{aligned}
|\mathcal{F}(u)| & =\int_{\Gamma}|\operatorname{tr}(u)|^{2} d x \\
& \leq \int_{\partial \Omega}|\operatorname{tr}(u)|^{2} d x \\
& \leq K\|u\|_{H^{1}(\Omega)}^{2}
\end{aligned}
$$

and hence $\mathcal{F}$ is a continuous functional on $H^{1}(\Omega)$. Also note that taking $\ell=1$, $\Pi_{\ell-1}=c$, where $c$ is any non-zero constant, and

$$
\mathcal{F}(c)=\int_{\Gamma}|\operatorname{tr}(c)|^{2} d x
$$

is non-zero if and only if the measure of $\Gamma$ is non-zero. Thus the norms generated by the inner products $(5.4),(5.5)$ are equivalent by Theorem 5.1 and there are finite, positive constants $M_{L}, M_{U}$ such that

$$
M_{L}\|\phi\|_{\mathcal{F}}^{2} \leq\|\phi\|_{\mathcal{V}}^{2} \leq M_{U}\|\phi\|_{\mathcal{F}}^{2}
$$

for all $\phi \in \mathcal{V}$ where

$$
\|\phi\|_{\mathcal{F}}^{2}=\|\nabla \phi\|_{\mathcal{H}}^{2}+\int_{\gamma_{2}}\left|\operatorname{Tr}_{2} \phi\right|^{2} d S_{2}
$$

is the norm generated by (5.5).

Define a sesquilinear form $\sigma: \mathcal{V} \times \mathcal{V} \rightarrow \mathbb{R}$ by

$$
\sigma(\phi, \psi)=\left\langle\frac{1}{g} k \nabla \phi, \nabla \psi\right\rangle_{\mathcal{H}}+h \int_{\gamma_{2}}\left(\operatorname{Tr}_{2} \phi\right)(z)\left(\operatorname{Tr}_{2} \psi\right)(z) d S_{2} .
$$


Note $\sigma$ defines an operator $A \in \mathscr{L}\left(\mathcal{V}, \mathcal{V}^{*}\right)$ where $\langle A \phi, \psi\rangle_{\mathcal{V}^{*}, \mathcal{V}}=\sigma(\phi, \psi)$ and $\mathscr{L}\left(\mathcal{V}, \mathcal{V}^{*}\right)$ is the set of all bounded, linear functionals from $\mathcal{V}$ to $\mathcal{V}^{*}$. This follows due to the continuity of $\sigma$ on $\mathcal{V} \times \mathcal{V}$ guaranteed by (5.6).

Define $F:[0, T] \rightarrow \mathcal{V}^{*}$ by

$$
[F(t)](\psi)=h T_{\infty} \int_{\gamma_{2}}\left(\operatorname{Tr}_{2} \psi\right)(z) d S_{2}+S_{0}(t) \int_{\gamma_{4}}\left(\operatorname{Tr}_{4} \psi\right)(z) d S_{4}
$$

for $\psi \in \mathcal{V}$.

\subsubsection{Weak Solution}

Suppose $u$ solves

$$
\dot{u}+A u=F \quad \text { in } \mathcal{V}^{*},
$$

i.e., for all $\psi \in \mathcal{V}$,

$$
\langle\dot{u}+A u-F, \psi\rangle_{\mathcal{V}^{*}, \mathcal{V}}=0 .
$$

So by definition,

$$
\begin{aligned}
&\langle\dot{u}(t), \psi\rangle_{\mathcal{V}^{*}, \mathcal{V}}=\langle-A u(t), \psi\rangle_{\mathcal{V}^{*}, \mathcal{v}}+\langle F, \psi\rangle_{\mathcal{V}^{*}, \mathcal{V}} \\
&=\left\langle-\frac{1}{g} k \nabla u(t), \nabla \psi\right\rangle_{\mathcal{H}}-h \int_{\gamma_{2}}\left(\operatorname{Tr}_{2} u(t)\right)(z)\left(\operatorname{Tr}_{2} \psi\right)(z) d S_{2} \\
& \quad+h T_{\infty} \int_{\gamma_{2}}\left(\operatorname{Tr}_{2} \psi\right)(z) d S_{2}+S_{0}(t) \int_{\gamma_{4}}\left(\operatorname{Tr}_{4} \psi\right)(z) d S_{4} \\
&=\langle-k \nabla u(t), \nabla \psi\rangle_{L^{2}}-h \int_{\gamma_{2}}\left(\left(\operatorname{Tr}_{2} u(t)\right)(z)-T_{\infty}\right)\left(\operatorname{Tr}_{2} \psi\right)(z) d S_{2} \\
& \quad+S_{0}(t) \int_{\gamma_{4}}\left(\operatorname{Tr}_{4} \psi\right)(z) d S_{4} .
\end{aligned}
$$

Now, if $u \in V$ and $k \nabla u \in V$, using the Divergence Theorem and the identity

$$
\nabla \cdot(k(z) \nabla s(t, z) \psi(z))=(\nabla \cdot(k(z) \nabla s(t, z)) \psi(z)+k(z)(\nabla s(t, z) \cdot \nabla \psi(z))
$$

in the previous equation, we see 


$$
\begin{aligned}
\langle\dot{u}(t), \psi\rangle_{\mathcal{V}^{*}, \mathcal{V}} & =\langle\nabla \cdot(k \nabla u(t)), \psi\rangle_{L^{2}}-\int_{\partial \Omega}\left(\operatorname{Tr}_{\Omega}(n \cdot k \nabla u(t))\right)(z)\left(\operatorname{Tr}_{\Omega} \psi\right)(z) d S \\
& -h \int_{\gamma_{2}}\left(\left(\operatorname{Tr}_{2} u(t)\right)(z)-T_{\infty}\right)\left(\operatorname{Tr}_{2} \psi\right)(z) d S_{2}+S_{0}(t) \int_{\gamma_{4}}\left(\operatorname{Tr}_{4} \psi\right)(z) d S_{4}
\end{aligned}
$$

where $\operatorname{Tr}_{\Omega}$ is the continuous trace operator mapping $f \in H^{1}(\Omega) \rightarrow H^{\frac{1}{2}}(\partial \Omega)$ with $\left(\operatorname{Tr}_{\Omega} f\right)(z)=\left.f(z)\right|_{\partial \Omega}$

If we have enough smoothness on $u$ with respect to the time derivative, i.e., $\dot{u} \in L^{2}(0, T ; \mathcal{H})$, then

$$
\langle\dot{u}(t), \psi\rangle_{\mathcal{V}^{*}, \mathcal{V}}=\langle\dot{u}(t), \psi\rangle_{\mathcal{H}}=\langle g \dot{u}(t), \psi\rangle_{L^{2}}
$$

and so

$$
\begin{aligned}
& \langle g \dot{u}(t)-\nabla \cdot(k \nabla u(t)), \psi\rangle_{L^{2}}+\int_{\partial \Omega}\left(\operatorname{Tr}_{\Omega}(n \cdot k \nabla u(t))\right)(z)\left(\operatorname{Tr}_{\Omega} \psi\right)(z) d S \\
& \quad+h \int_{\gamma_{2}}\left(\left(\operatorname{Tr}_{2} u(t)\right)(z)-T_{\infty}\right)\left(\operatorname{Tr}_{2} \psi\right)(z) d S_{2}-S_{0}(t) \int_{\gamma_{4}}\left(\operatorname{Tr}_{4} \psi\right)(z) d S_{4}=0
\end{aligned}
$$

for all $\psi \in \mathcal{V}$.

We know (5.11) holds for all $\psi \in \mathcal{V}=H^{1}(\Omega)$, thus for all $\psi \in H_{0}^{1}(\Omega)$,

$$
\langle g \dot{u}(t)-\nabla \cdot(k \nabla u(t)), \psi\rangle_{L^{2}}=0 .
$$

Since $H_{0}^{1}(\Omega)$ is a dense subset of $L^{2}(\Omega)$, we know $g \dot{u}(t)-\nabla \cdot(k \nabla u(t))=0$ in the $L^{2}$ sense. Thus we can rewrite (5.11) as

$$
\begin{aligned}
\int_{\partial \Omega}\left(\operatorname{Tr}_{\Omega}(n \cdot k \nabla u(t))\right)(z)\left(\operatorname{Tr}_{\Omega} \psi\right)(z) d S \\
+h \int_{\gamma_{2}}\left(\left(\operatorname{Tr}_{2} u(t)\right)(z)-T_{\infty}\right)\left(\operatorname{Tr}_{2} \psi\right)(z) d S_{2} \\
\quad-S_{0}(t) \int_{\gamma_{4}}\left(\operatorname{Tr}_{4} \psi\right)(z) d S_{4}=0
\end{aligned}
$$

for all $\psi \in \mathcal{V}$.

We know $\partial \Omega=\gamma_{1} \cup \gamma_{2} \cup \gamma_{3} \cup \gamma_{4}$. Thus for $\psi \in H_{\gamma_{1}} \equiv\left\{\phi \in H^{1}(\Omega):\left.\phi\right|_{\gamma_{2}, \gamma_{3}, \gamma_{4}}=0\right\} \subset$ $H^{1}(\Omega)$, we see

$$
\int_{\partial \Omega}\left(\operatorname{Tr}_{\Omega}(n \cdot k \nabla u(t))\right)(z)\left(\operatorname{Tr}_{\Omega} \psi\right)(z) d S=\int_{\gamma_{1}}\left(\operatorname{Tr}_{1}(n \cdot k \nabla u(t))\right)(z)\left(\operatorname{Tr}_{1} \psi\right)(z) d S_{1}
$$


and so using (5.12) we see $\left.k(z) \frac{\partial u}{\partial n}(t, z)\right|_{\gamma_{1}}=0$. A similar argument shows $\left.k(z) \frac{\partial u}{\partial n}(t, z)\right|_{\gamma_{3}}=0$.

For $\psi \in H_{\gamma_{2}} \equiv\left\{\phi \in H^{1}(\Omega):\left.\phi\right|_{\gamma_{1}, \gamma_{3}, \gamma_{4}}=0\right\} \subset H^{1}(\Omega)$, using (5.12) we see

$$
\begin{aligned}
& \int_{\gamma_{2}}\left(\operatorname{Tr}_{2}(n \cdot k \nabla u(t))\right)(z)\left(\operatorname{Tr}_{2} \psi\right)(z) d S_{2} \\
& +h \int_{\gamma_{2}}\left(\left(\operatorname{Tr}_{2} u(t)\right)(z)-T_{\infty}\right)\left(\operatorname{Tr}_{2} \psi\right)(z) d S_{2}=0
\end{aligned}
$$

and thus $\left.k(z) \frac{\partial u}{\partial n}(t, z)\right|_{\gamma_{2}}=\left.h\left(T_{\infty}-u(t, z)\right)\right|_{\gamma_{2}}$.

For $\psi \in H_{\gamma_{4}} \equiv\left\{\phi \in H^{1}(\Omega):\left.\phi\right|_{\gamma_{1}, \gamma_{2}, \gamma_{3}}=0\right\} \subset H^{1}(\Omega)$, using (5.12) we see

$$
\int_{\gamma_{4}}\left(\operatorname{Tr}_{4}(n \cdot k \nabla u(t))\right)(z)\left(\operatorname{Tr}_{4} \psi\right)(z) d S_{4}-S_{0}(t) \int_{\gamma_{4}}\left(\operatorname{Tr}_{4} \psi\right)(z) d S_{4}=0
$$

and thus $\left.\left.k(z) \frac{\partial u}{\partial n}(t, z)\right|_{\gamma_{4}}=S_{0}(t)\right)\left.\right|_{\gamma_{4}}$. Thus, any solution of (5.10) that has sufficient additional smoothness is a strong solution of (5.1).

Thus we have shown a solution $u$ of (5.10) (if it exists) is a weak solution of (5.1). That is, (5.10) is the weak form of (5.1).

\subsection{Well-Posedness}

\subsubsection{Existence and Uniqueness}

We want to show existence of solutions to parabolic systems of the form

$$
\left\{\begin{array}{l}
\dot{u}+A u=F \quad \text { in } \mathcal{V}^{*} \\
u(0)=u_{0}
\end{array}\right.
$$

We will use the Gelfand triple $\mathcal{V} \hookrightarrow \mathcal{H} \cong \mathcal{H}^{*} \hookrightarrow \mathcal{V}^{*}$ where the embedding $\mathcal{V} \hookrightarrow \mathcal{H}$ is dense and continuous with $\|\phi\|_{\mathcal{H}} \leq c\|\phi\|_{\mathcal{\nu}}$ for all $\phi \in \mathcal{V}$ and some finite constant $c>0$. Note the desired inequality holds:

$$
\begin{aligned}
\|\phi\|_{\mathcal{V}}^{2} & =\langle\nabla \phi, \nabla \phi\rangle_{\mathcal{H}}+\langle\phi, \phi\rangle_{L^{2}} \\
& \geq\langle\phi, \phi\rangle_{L^{2}}
\end{aligned}
$$




$$
\begin{aligned}
& =\left\langle\frac{1}{g} \phi, \phi\right\rangle_{\mathcal{H}} \\
& \geq R_{U}^{-1}\|\phi\|_{\mathcal{H}}^{2}
\end{aligned}
$$

so in fact $\|\phi\|_{\mathcal{H}} \leq \sqrt{R_{U}}\|\phi\|_{\mathcal{v}}$.

We shall argue and then use two standard conditions on $\sigma$ defined by (5.8):

(1) The form $\sigma$ is $\mathcal{V}$-bounded: for all $\phi, \psi \in \mathcal{V}$, there exists a $B<\infty$ such that

$$
|\sigma(\phi, \psi)| \leq B\|\phi\|_{\mathcal{v}}\|\psi\|_{\mathcal{v}}
$$

(2) The form $\sigma$ is $\mathcal{V}$-coercive: for all $\phi \in \mathcal{V}$, there exists a $C>0$ such that

$$
|\sigma(\phi, \phi)| \geq C\|\phi\|_{\mathcal{v}}^{2}
$$

We also find the forcing term $F$ defined by (5.9) satisfies

$$
F \in L^{2}\left(0, T ; \mathcal{V}^{*}\right)
$$

To show that $\sigma$ of (5.8) satisfies (5.14), we use (5.2), (5.3), and (5.7):

$$
\begin{aligned}
|\sigma(\phi, \psi)| & =\left|\left\langle\frac{1}{g} k \nabla \phi, \nabla \psi\right\rangle_{\mathcal{H}}+h \int_{\gamma_{2}}\left(\operatorname{Tr}_{2} \phi\right)(z)\left(\operatorname{Tr}_{2} \psi\right)(z) d S_{2}\right| \\
& \leq R_{L}^{-1} K_{U}\|\nabla \phi\|_{\mathcal{H}}\|\nabla \psi\|_{\mathcal{H}}+h\left\|\operatorname{Tr}_{2} \phi\right\|_{\gamma_{2}}\left\|\operatorname{Tr}_{2} \psi\right\|_{\gamma_{2}} \\
& \leq \max \left\{R_{L}^{-1} K_{U}, h\right\}\left(\|\nabla \phi\|_{\mathcal{H}}\|\nabla \psi\|_{\mathcal{H}}+\left\|\operatorname{Tr}_{2} \phi\right\|_{\gamma_{2}}\left\|\operatorname{Tr}_{2} \psi\right\|_{\gamma_{2}}\right) \\
& \leq \max \left\{R_{L}^{-1} K_{U}, h\right\}\left(\|\nabla \phi\|_{\mathcal{H}}+\left\|\operatorname{Tr}_{2} \phi\right\|_{\gamma_{2}}\right)\left(\|\nabla \psi\|_{\mathcal{H}}+\left\|\operatorname{Tr}_{2} \psi\right\|_{\gamma_{2}}\right) \\
& \leq \max \left\{R_{L}^{-1} K_{U}, h\right\}\left(2\|\phi\|_{\mathcal{F}}\right)\left(2\|\psi\|_{\mathcal{F}}\right) \\
& \leq 4 M_{L}^{-1} \max \left\{R_{L}^{-1} K_{U}, h\right\}\|\phi\| \nu\|\psi\|_{\mathcal{\nu}}
\end{aligned}
$$

where $\|f\|_{\gamma_{2}}^{2}=\int_{\gamma_{2}}|f(z)|^{2} d S_{2}$ for any $f \in H^{\frac{1}{2}}\left(\gamma_{2}\right)$. Thus $\sigma$ is $\mathcal{V}$-bounded. 
Similarly, for (5.15) we have the following:

$$
\begin{aligned}
\sigma(\phi, \phi) & =\left\langle\frac{1}{g} k \nabla \phi, \nabla \phi\right\rangle_{\mathcal{H}}+h \int_{\gamma_{2}}\left(\operatorname{Tr}_{2} \phi\right)(z)\left(\operatorname{Tr}_{2} \phi\right)(z) d S_{2} \\
& \geq R_{U}^{-1} K_{L}\|\nabla \phi\|_{\mathcal{H}}^{2}+h \int_{\gamma_{2}}\left|\left(\operatorname{Tr}_{2} \phi\right)(z)\right|^{2} d S_{2} \\
& \geq \min \left\{R_{U}^{-1} K_{L}, h\right\}\left(\|\nabla \phi\|_{\mathcal{H}}^{2}+\int_{\gamma_{2}}\left|\left(\operatorname{Tr}_{2} \phi\right)(z)\right|^{2} d S_{2}\right) \\
& \geq \min \left\{R_{U}^{-1} K_{L}, h\right\} M_{U}^{-1}\|\phi\|_{\mathcal{V}}^{2}
\end{aligned}
$$

so $\sigma$ is $\mathcal{V}$-coercive.

In order to see that (5.16) holds, recall $T r_{2}: \mathcal{V}=H^{1}(\Omega) \rightarrow H^{\frac{1}{2}}\left(\gamma_{2}\right)$. So, for any $\psi \in \mathcal{V}, \operatorname{Tr}_{2} \psi \in H^{\frac{1}{2}}\left(\gamma_{2}\right) \subset L^{2}(\Omega)$, and hence $\int_{\gamma_{2}} \operatorname{Tr}_{2} \psi d S_{2} \in \mathbb{R}$. Thus $\psi \rightarrow$ $\int_{\gamma_{2}} T r_{2} \psi d S_{2}$ is a continuous mapping from $\mathcal{V} \rightarrow \mathbb{R}$, i.e., it is in $\mathcal{V}^{*}$. If $S_{0} \in L^{2}(0, T)$ (which it does in our problem), then $F \in L^{2}\left(0, T ; \mathcal{V}^{*}\right)$.

Given the above hypothesis, the system (5.13) is equivalently written

$$
\left\{\begin{array}{l}
\langle\dot{u}(t), \psi\rangle+\sigma(u(t), \psi)=\langle F(t), \psi\rangle \\
u(0)=u_{0}
\end{array}\right.
$$

for $\psi \in \mathcal{V}$ where the duality product $\langle\cdot, \cdot\rangle$ is $\langle\cdot, \cdot\rangle_{\mathcal{V}^{*}, \mathcal{V}}$.

Assume for the moment that (5.17) has a solution $u$. We derive an a priori bound. Let $\psi=u(t)$ for a fixed $t$. Substituting into (5.17) we obtain

$$
\langle\dot{u}(t), u(t)\rangle_{\mathcal{V}^{*}, \mathcal{V}}+\sigma(u(t), u(t))=\langle F(t), u(t)\rangle_{\mathcal{V}^{*}, \mathcal{V}}
$$

and since $\langle\dot{u}(t), u(t)\rangle_{\mathcal{V}^{*}, \mathcal{V}}=\frac{1}{2} \frac{d}{d t}\left\{\|u(t)\|_{\mathcal{H}}^{2}\right\}$ we see

$$
\frac{1}{2} \frac{d}{d t}\left\{\|u(t)\|_{\mathcal{H}}^{2}\right\}+\sigma(u(t), u(t))=\langle F(t), u(t)\rangle_{\mathcal{V}^{*}, \mathcal{V}}
$$

for any $t$ in a given interval $[0, T]$. Integrating from 0 to $t$ we have

$$
\int_{0}^{t}\left\{\frac{1}{2} \frac{d}{d \xi}\left\{\|u(\xi)\|_{\mathcal{H}}^{2}\right\}+\sigma(u(\xi), u(\xi))\right\} d \xi=\int_{0}^{t}\langle F(\xi), u(\xi)\rangle_{\mathcal{V}^{*}, \mathcal{V}} d \xi
$$


and so

$$
\frac{1}{2}\|u(t)\|_{\mathcal{H}}^{2}-\frac{1}{2}\|u(0)\|_{\mathcal{H}}^{2}+\int_{0}^{t} \sigma(u(\xi), u(\xi)) d \xi=\int_{0}^{t}\langle F(\xi), u(\xi)\rangle_{\mathcal{V}^{*}, \mathcal{V}} d \xi
$$

Using (5.15), the Cauchy Schwartz inequality and the fact $2 a b \leq a^{2}+b^{2}$ we have

$$
\begin{aligned}
\|u(t)\|_{\mathcal{H}}^{2}+2 C \int_{0}^{t}\|u(\xi)\|_{\mathcal{V}}^{2} d \xi & \leq\left\|u_{0}\right\|_{\mathcal{H}}^{2}+2\left|\int_{0}^{t}\langle F(\xi), u(\xi)\rangle_{\mathcal{V}^{*}, \mathcal{V}} d \xi\right| \\
& \leq\left\|u_{0}\right\|_{\mathcal{H}^{\prime}}^{2}+2 \int_{0}^{t}\|F(\xi)\| \mathcal{V}^{*}\|u(\xi)\|_{\mathcal{V}} d \xi \\
& \leq\left\|u_{0}\right\|_{\mathcal{H}}^{2}+\frac{1}{C} \int_{0}^{t}\|F(\xi)\|_{\mathcal{V}^{*}}^{2} d \xi+C \int_{0}^{t}\|u(\xi)\|_{\mathcal{V}}^{2} d \xi
\end{aligned}
$$

so

$$
\|u(t)\|_{\mathcal{H}}^{2}+C \int_{0}^{t}\|u(\xi)\|_{\mathcal{V}}^{2} d \xi \leq\left\|u_{0}\right\|_{\mathcal{H}^{\mathcal{C}}}^{2}+\frac{1}{C} \int_{0}^{t}\|F(\xi)\|_{\mathcal{V}^{*}}^{2} d \xi
$$

Thus

$$
\|u(t)\|_{\mathcal{H}}^{2}+C \int_{0}^{t}\|u(\xi)\|_{\mathcal{V}}^{2} d \xi \leq \hat{C}
$$

where $\hat{C}=\hat{C}\left(\left\|u_{0}\right\|_{\mathcal{H}}, C,\|F\|_{L^{2}\left(0, T ; \mathcal{V}^{*}\right)}\right)$.

Let $\left\{\psi_{j}\right\}_{j=1}^{\infty} \subseteq \mathcal{V}$ be a linearly independent total subset of $\mathcal{V}$. Define a "Galerkin" approximation for (5.13) by

$$
u_{n}(t)=\sum_{j=1}^{n} c_{n j}(t) \psi_{j}
$$

where the coefficients $\left\{c_{n j}(t)\right\}_{j=1}^{n}$ are chosen so that $u_{n}(t)$ is the unique solution of the $n$-dimensional system

$$
\left\langle\dot{u}_{n}(t), \psi_{j}\right\rangle_{\mathcal{V}^{*}, \mathcal{V}}+\sigma\left(u_{n}(t), \psi_{j}\right)=\left\langle F(t), \psi_{j}\right\rangle_{\mathcal{V}^{*}, \mathcal{V}}
$$

for $j=1, \cdots, n$ satisfying the initial condition $c_{n j}(0)=c_{n j 0}$. The set $\left\{c_{n j 0}\right\}$ is chosen so that

$$
u_{0}=\lim _{n \rightarrow \infty} \sum_{j=1}^{n} c_{n j 0} \psi_{j}
$$

in $V$. Define $u_{n 0}=u_{n}(0)$. 
Multiplying (5.18) by $c_{n j}(t)$ and summing over $j=1, \cdots, n$ we obtain

$$
\left\langle\dot{u}_{n}(t), u_{n}(t)\right\rangle+\sigma\left(u_{n}(t), u_{n}(t)\right)=\left\langle F(t), u_{n}(t)\right\rangle_{\mathcal{V}^{*}, \mathcal{V}}
$$

Repeating the same argument as above we see

$$
\left\|u_{n}(t)\right\|_{\mathcal{H}}^{2}+C \int_{0}^{t}\left\|u_{n}(\xi)\right\|_{\mathcal{V}}^{2} d \xi \leq\left\|u_{n 0}\right\|_{\mathcal{H}}^{2}+\frac{1}{C} \int_{0}^{t}\|F(\xi)\|_{\mathcal{V}^{*}}^{2} d \xi .
$$

Since the convergence $u_{n 0} \rightarrow u_{0}$ guarantees uniform boundedness of $\left|u_{n 0}\right|_{\mathcal{H}}$ we see

$$
\left\|u_{n}(t)\right\|_{\mathcal{H}}^{2}+C \int_{0}^{t}\left\|u_{n}(\xi)\right\|_{\mathcal{V}}^{2} d \xi \leq \tilde{C}
$$

where $\tilde{C}=\tilde{C}\left(\left\|u_{0}\right\|_{\mathcal{H}}, C,\|F\|_{L^{2}\left(0, T ; \mathcal{V}^{*}\right)}\right)$.

Lemma 5.2 There exists a subsequence $\left\{u_{n}\right\}$ of the original sequence of Galerkin approximations and $u \in L^{2}(0, T ; \mathcal{V})$ such that

(i) $u_{n} \rightarrow u$ in $L^{2}(0, T ; \mathcal{V})$

(ii) $u_{n}(t) \rightarrow u(t)$ in $\mathcal{H}$ a.e. in $t \in[0, T]$

Here $\rightarrow$ denotes weak convergence. Any such subsequence will be denoted $\left\{u_{n}\right\}$.

Proof:

i) We know $\left\{u_{n}\right\}$ is bounded in $L^{2}(0, T ; \mathcal{V})$ by $(5.19)$. Thus, there exists a subsequence that converges weakly to $u$ in $L^{2}(0, T ; \mathcal{V})$.

ii) From (5.19), we know $\left\|u_{n}(t)\right\|_{\mathcal{H}}^{2} \leq \tilde{C}$, where $\tilde{C}$ is independent of $t$. Thus

$$
\begin{aligned}
\left\|u_{n}\right\|_{L^{2}(0, T ; \mathcal{H})}^{2} & =\int_{0}^{T}\left\|u_{n}(s)\right\|_{\mathcal{H}} d s \\
& \leq \int_{0}^{T} \tilde{C} d s \\
& =T \tilde{C} .
\end{aligned}
$$

Since both $T$ and $\tilde{C}$ are finite constants, this says $\left\|u_{n}\right\|_{L^{2}(0, T ; \mathcal{H})}$ is a bounded sequence, and hence there is a subsequence which converges weakly to $u$ in $L^{2}(0, T ; \mathcal{H})$; i.e. there exists a subsequence $\left\{u_{n}(t)\right\}$ which converges weakly in $\mathcal{H}$ to $u(t)$ a.e. in $t \in[0, T]$. 
Theorem 5.3 Under assumptions (5.14), (5.15), and (5.16), for $u_{0} \in V$, there exists a weak solution of (5.13) with $u \in L^{2}(0, T ; \mathcal{V})$ and $\dot{u} \in L^{2}\left(0, T ; \mathcal{V}^{*}\right)$. Furthermore, this solution is unique.

Proof: Let $P_{M}(M=1,2, \cdots)$ be the class of functions $\eta \in L^{2}(0, T ; \mathcal{V})$ which can be represented in the form

$$
\eta(t)=\sum_{j=1}^{M} a_{j}(t) \psi_{j}
$$

where $a_{j} \in C^{1}(0, T)$ and $\left\{\psi_{j}\right\}_{j=1}^{\infty} \subseteq \mathcal{V}$ is a linearly independent total subset of $\mathcal{V}$. Let $P=\cup_{M=1}^{\infty} P_{M}$. Note $P$ is dense in $L^{2}(0, T ; \mathcal{V})$.

Multiply the $j^{\text {th }}$ equation in (5.18) by $a_{j}(t)$ and sum from 1 to $M$; this yields

$$
\left\langle\dot{u}_{n}(t), \eta(t)\right\rangle_{\mathcal{V}^{*}, \mathcal{V}}+\sigma\left(u_{n}(t), \eta(t)\right)=\langle F(t), \eta(t)\rangle_{\mathcal{V}^{*}, \mathcal{V}}
$$

Integrating from 0 to $t$ we obtain

$$
\int_{0}^{t}\left\{\left\langle\dot{u}_{n}(\xi), \eta(\xi)\right\rangle_{\mathcal{V}^{*}, \mathcal{V}}+\sigma\left(u_{n}(\xi), \eta(\xi)\right)\right\} d \xi=\int_{0}^{t}\langle F(\xi), \eta(\xi)\rangle_{\mathcal{V}^{*}, \mathcal{V}} d \xi
$$

Integrating by parts in the first term we have

$$
\begin{aligned}
\int_{0}^{t}\left\{-\left\langle u_{n}(\xi), \dot{\eta}(\xi)\right\rangle_{\mathcal{V}^{*}, \mathcal{V}}+\sigma\right. & \left.\left(u_{n}(\xi), \eta(\xi)\right)\right\} d \xi+\left\langle u_{n}(t), \eta(t)\right\rangle_{\mathcal{V}^{*}, \mathcal{V}} \\
= & \left\langle u_{n}(0), \eta(0)\right\rangle_{\mathcal{V}^{*}, \mathcal{V}}+\int_{0}^{t}\langle F(\xi), \eta(\xi)\rangle_{\mathcal{V}^{*}, \mathcal{V}} d \xi
\end{aligned}
$$

for all $\eta \in P_{M}, M \leq n$.

Fix $\eta \in P_{M}$. Note $\eta \in C^{1}(0, T ; \mathcal{V})$ and $u_{n}(\xi) \in \mathcal{V}$ for all $\xi \in[0, T]$. Thus

$$
\left\langle u_{n}(\xi), \dot{\eta}(\xi)\right\rangle_{\mathcal{V}^{*}, \mathcal{v}}=\left\langle u_{n}(\xi), \dot{\eta}(\xi)\right\rangle_{\mathcal{H}}
$$

and

$$
\left\langle u_{n}(t), \eta(t)\right\rangle_{\mathcal{V}^{*}, \mathcal{V}}=\left\langle u_{n}(t), \eta(t)\right\rangle_{\mathcal{H}}
$$

for all $t \in[0, T]$. All statements in Lemma 5.2 hold for any interval $[0, t] \subset[0, T]$, so there exists a subsequence $\left\{u_{n}\right\}$ satisfying both $(i)$ and $(i i)$ that satisfies

$$
\int_{0}^{t}-\left\langle u_{n}(\xi), \dot{\eta}(\xi)\right\rangle_{\mathcal{H}} d \xi \rightarrow \int_{0}^{t}-\langle u(\xi), \dot{\eta}(\xi)\rangle_{\mathcal{H}} d \xi
$$


and

$$
\left\langle u_{n}(t), \dot{\eta}(t)\right\rangle_{\mathcal{H}} \rightarrow\langle u(t), \dot{\eta}(t)\rangle_{\mathcal{H}} \quad \text { a.e. } t \in[0, T]
$$

Since $u_{n 0} \rightarrow u_{0}$ strongly,

$$
\left\langle u_{n}(0), \dot{\eta}(0)\right\rangle_{\mathcal{H}} \rightarrow\left\langle u_{0}, \dot{\eta}(0)\right\rangle_{\mathcal{H}}
$$

Finally, since $\sigma$ is $\mathcal{V}$-bounded, the map $u \rightarrow \int_{0}^{t} \sigma(u(\xi), \eta(\xi)) d \xi$ is a bounded linear functional on $L^{2}(0, T ; \mathcal{V})$. Thus it is weakly continuous and so

$$
\int_{0}^{t} \sigma\left(u_{n}(\xi), \eta(\xi)\right) d \xi \rightarrow \int_{0}^{t} \sigma(u(\xi), \eta(\xi)) d \xi
$$

Thus

$$
\begin{gathered}
\int_{0}^{t}\left\{-\langle u(\xi), \dot{\eta}(\xi)\rangle_{\mathcal{H}}+\sigma(u(\xi), \eta(\xi))\right\} d \xi+\langle u(t), \eta(t)\rangle_{\mathcal{H}} \\
=\left\langle u_{0}, \eta(0)\right\rangle_{\mathcal{H}}+\int_{0}^{t}\langle F(\xi), \eta(\xi)\rangle_{\mathcal{V}^{*}, \mathcal{V}} d \xi
\end{gathered}
$$

for all $\eta \in P_{M}$ and a.e. in $t$.

Now for each $j$ choose $\eta_{j}(t)=a(t) \psi_{j}$ for arbitrary $a \in C_{0}^{\infty}(0, T)$. From (5.20) we see

$$
\int_{0}^{t} \dot{a}(\xi)\left\langle-u(\xi), \psi_{j}\right\rangle_{\mathcal{H}} d \xi+\int_{0}^{t} a(\xi)\left\{\sigma\left(u(\xi), \psi_{j}\right)-\left\langle F(\xi), \psi_{j}\right\rangle_{\mathcal{V}^{*}, \mathcal{v}}\right\} d \xi=0 .
$$

For $u \in L^{2}(0, T ; \mathcal{V}), \psi \in \mathcal{V}$, and $a \in C_{0}^{\infty}(0, T)$, let

$$
\left.L(u, a \psi) \equiv \int_{0}^{T} a(\xi)\left\{\sigma(u(\xi), \psi)-\langle F(\xi), \psi\rangle_{\mathcal{V}^{*}, \mathcal{v}}\right\}\right\} d \xi .
$$

Then we have shown for all $\psi_{j}$ and any $a \in C_{0}^{\infty}(0, T)$, we have

$$
\int_{0}^{T}\left\langle u(\xi), \psi_{j}\right\rangle_{\mathcal{H}} \dot{a}(\xi) d \xi=L\left(u, a \psi_{j}\right) .
$$

Since both the left and right sides of this equation are linear in $\psi_{j}$ and continuous in the $\mathcal{V}$ topology and $\left\{\psi_{j}\right\}$ are total in $\mathcal{V}$, we see by continuity and density that (5.21) implies

$$
\int_{0}^{T}\langle u(\xi), \psi\rangle_{\mathcal{H}} \dot{a}(\xi) d \xi=L(u, a \psi)
$$


for all $\psi \in \mathcal{V}$ and $a \in C_{0}^{\infty}(0, T)$. Thus

$$
\int_{0}^{T}\langle u(\xi), \psi\rangle_{\mathcal{V}^{*}, \mathcal{V}} \dot{a}(\xi) d \xi=\int_{0}^{T}\langle A u(\xi)-F(\xi), \psi\rangle_{\mathcal{V}^{*}, \mathcal{V}} a(\xi) d \xi
$$

for all $\psi \in \mathcal{V}$ and all $a \in C_{0}^{\infty}(0, T)$. That is, for

$$
G^{u, \psi}(\xi) \equiv\langle A u(\xi)-F(\xi), \psi\rangle_{\mathcal{V}^{*}, \mathcal{V}}
$$

and

$$
f^{u, \psi}(\xi) \equiv\langle u(\xi), \psi\rangle_{\mathcal{V}^{*}, \mathcal{V}}
$$

we have

$$
\int_{0}^{T} f^{u, \psi}(\xi) \dot{a}(\xi) d \xi=\int_{0}^{T} G^{u, \psi}(\xi) a(\xi) d \xi
$$

for all $a \in C_{0}^{\infty}(0, T)$. But note that

$$
a \rightarrow \int_{0}^{T} G^{u, \psi}(\xi) a(\xi) d \xi=\mathcal{L}^{u, \psi}(a)
$$

is in $L^{2}(0, T) *$ with

$$
\left\langle f^{u, \psi}, \dot{a}\right\rangle_{L^{2}(0, T)}=\mathcal{L}^{u, \psi}(a)
$$

for all $a \in C_{0}^{\infty}(0, T)$. Thus $\frac{d}{d t}\left\{f^{u, \psi}\right\}$ exists in $L^{2}(0, T)^{*} \simeq L^{2}(0, T)$ and so $\frac{d}{d t}\left\{f^{u, \psi}\right\}=$ $-G^{u, \psi}$ in the $L^{2}(0, T)$ sense. In other words, in the $L^{2}(0, T)$ sense we have

$$
\lim _{h \rightarrow 0}\left\langle\frac{u(\xi+h)-u(\xi)}{h}, \psi\right\rangle_{\mathcal{V}^{*}, \mathcal{V}}=\langle-A u(\xi)+F(\xi), \psi\rangle_{\mathcal{V}^{*}, \mathcal{V}} .
$$

This says

$$
\lim _{h \rightarrow 0} \frac{u(\xi+h)-u(\xi)}{h}
$$

exists in the $\mathcal{V}^{*}$ sense (in the $L^{2}(0, T)^{*} \simeq L^{2}(0, T)$ sense). Thus $\dot{u} \in L^{2}\left(0, T ; \mathcal{V}^{*}\right)$ and for all $\psi \in \mathcal{V}$,

$$
\langle\dot{u}, \psi\rangle_{\mathcal{V}^{*}, \mathcal{V}}=\langle-A u(\xi)+F(\xi), \psi\rangle_{\mathcal{V}^{*}, \mathcal{V}}
$$

in the $L^{2}(0, T)$ sense. 
To see that $u(0)=u_{0}$ we return to (5.20) which holds for each $\eta(t)=a(t) \psi_{j}$ where $a \in C^{1}(0, T), a(0) \neq 0$. Integrating by parts and using (5.22) we see

$$
\langle u(t), \eta(t)\rangle_{\mathcal{V}^{*}, \mathcal{V}}+\left.\langle-u(\xi), \eta(\xi)\rangle_{\mathcal{V}^{*}, \mathcal{V}}\right|_{\xi=0} ^{t}=\left\langle u_{0}, \eta(0)\right\rangle_{\mathcal{V}^{*}, \mathcal{V}}
$$

and substituting $\eta(0)=a(0) \psi_{j}$ shows

$$
a(0)\left\langle u(0), \psi_{j}\right\rangle \mathcal{V}^{*}, \mathcal{v}=a(0)\left\langle u_{0}, \psi_{j}\right\rangle_{\mathcal{V}^{*}, \mathcal{V}}
$$

for all $j$ and so $u_{0}=u(0)$. Thus $u(t)$ is a weak solution of (5.13).

In order to see that the solution $u$ is unique, let $w_{1}\left(\cdot ; u_{0}, F\right), w_{2}\left(\cdot ; u_{0}, F\right)$ be two solutions of (5.17). Note $v \equiv w_{1}-w_{2}$ satisfies $v(0)=0$ and

$$
\langle\dot{v}(\xi), \eta\rangle \mathcal{v}^{*}, \mathcal{v}+\sigma(v(\xi), \eta)=0
$$

for all $\eta \in \mathcal{V}$. For fixed $\xi \in[0, T]$, let $\eta=v(\xi)$ in the above expression. Integrating from 0 to $t$ we have

$$
\int_{0}^{t}\left\{\langle\dot{v}(\xi), v(\xi)\rangle_{\mathcal{v}^{*}, \mathcal{v}}+\sigma(v(\xi), v(\xi))\right\} d \xi=0
$$

Since $\langle\dot{v}(\xi), v(\xi)\rangle_{\mathcal{V}^{*}, \mathcal{V}}=\frac{1}{2} \frac{d}{d t}\left\{\|v(\xi)\|_{\mathcal{H}}^{2}\right\}$ we know

$$
\|v(t)\|_{\mathcal{H}}^{2}+2 \int_{0}^{t} \sigma(v(\xi), v(\xi)) d \xi=0
$$

and since $\sigma$ is $\mathcal{V}$-coercive

$$
\|v(t)\|_{\mathcal{H}}^{2}+2 C \int_{0}^{t}\|v(\xi)\|_{\mathcal{V}}^{2} d \xi \leq\|v(t)\|_{\mathcal{H}}^{2}+2 \int_{0}^{t} \sigma(v(\xi), v(\xi)) d \xi=0
$$

This yields

$$
\|v(t)\|_{\mathcal{H}}^{2}+2 C \int_{0}^{t}\|v(\xi)\|_{\mathcal{V}}^{2} d \xi=0
$$

and hence $\|v(t)\|_{\mathcal{H}}^{2}=0$. Thus $v(t)=0$ on any interval in $[0, T]$ and hence the solution is unique. 


\subsubsection{Continuous Dependence on Initial Data and Forcing Function}

Suppose $u=u\left(\cdot ; u_{0}, F\right)$ is a weak solution of

$$
\left\{\begin{array}{l}
\dot{u}+A u=F \\
u(0)=u_{0}
\end{array}\right.
$$

and suppose $u_{n}=u_{n}\left(\cdot ; u_{n 0}, F_{n}\right)$ is a weak solution of

$$
\left\{\begin{array}{l}
\dot{u}_{n}+A u_{n}=F_{n} \\
u_{n}(0)=u_{n 0}
\end{array}\right.
$$

with $u_{n 0} \rightarrow u_{0}$ in $\mathcal{H}$ and $F_{n} \rightarrow F$ in $L^{2}\left(0, T ; \mathcal{V}^{*}\right)$. Given systems (5.23) and (5.24) we see

$$
\left\{\begin{array}{l}
\left\langle\dot{u}-\dot{u}_{n}, \psi\right\rangle+\sigma\left(u-u_{n}, \psi\right)=\left\langle F(t)-F_{n}(t), \psi\right\rangle \\
u(0)-u_{n}(0)=u_{0}-u_{n 0}
\end{array}\right.
$$

for all $\psi \in \mathcal{V}$. If we let $\psi=u(t)-u_{n}(t)$ in (5.25) and use the same arguments as in Section 5.2.1, we see

$\left\|u(t)-u_{n}(t)\right\|_{\mathcal{H}}^{2}+C \int_{0}^{t}\left\|u(\xi)-u_{n}(\xi)\right\|_{\mathcal{V}}^{2} d \xi \leq\left\|u_{0}-u_{n 0}\right\|_{\mathcal{H}^{+}}^{2}+\frac{1}{C} \int_{0}^{t}\left\|F(\xi)-F_{n}(\xi)\right\|_{\mathcal{V}^{*}}^{2} d \xi$

and so

$$
\left\|u(t)-u_{n}(t)\right\|_{\mathcal{H}}^{2}+C \int_{0}^{t}\left\|(\xi)-u_{n}(\xi)\right\|_{\mathcal{V}}^{2} d \xi \leq\left\|u_{0}-u_{n 0}\right\|_{\mathcal{H}}^{2}+\frac{1}{C}\left\|F-F_{n}\right\|_{L^{2}\left(0, T ; \mathcal{V}^{*}\right)}
$$

Thus given that $u_{n 0} \rightarrow u_{0}$ in $\mathcal{H}$ and $F_{n} \rightarrow F$ in $L^{2}\left(0, T ; \mathcal{V}^{*}\right)$, we see $u \rightarrow u_{n}$ in $C(0, T ; \mathcal{H})$ and also in $L^{2}(0, T ; \mathcal{V})$. In fact, we have proved the following theorem:

Theorem 5.4 The mapping $\left(u_{0}, F\right) \rightarrow u\left(\cdot ; u_{0}, F\right)$, where $u\left(\cdot ; u_{0}, F\right)$ is a solution to (5.23) is continuous from $\mathcal{H} \times L^{2}\left(0, T ; \mathcal{V}^{*}\right)$ to $C(0, T ; \mathcal{H}) \cap L^{2}(0, T ; \mathcal{V})$.

Thus the solution depends continuously on the initial data and the forcing function and hence the problem is well-posed. 


\subsection{Continuous Dependence on Parameters}

\subsubsection{Continuous Dependence on $k$ and $h$}

Suppose $u(\cdot ; k, h)$ is a weak solution of

$$
\left\{\begin{array}{l}
\dot{u}+A u=F \\
u(0)=u_{0}
\end{array}\right.
$$

and let $u_{n}\left(\cdot ; k_{n}, h_{n}\right)$ be a weak solution of

$$
\left\{\begin{array}{l}
\dot{u}_{n}+A_{n} u_{n}=F_{n} \\
u_{n}(0)=u_{0}
\end{array}\right.
$$

where

$$
\left\langle A_{n} \phi, \psi\right\rangle_{\mathcal{V}^{*}, \mathcal{V}} \equiv \sigma_{n}(\phi, \psi)=\left\langle\frac{1}{g} k_{n} \nabla \phi, \nabla \psi\right\rangle_{\mathcal{H}}+h_{n} \int_{\gamma_{2}}\left(\operatorname{Tr}_{2} \phi\right)(z)\left(\operatorname{Tr}_{2} \psi\right)(z) d S_{2}
$$

and

$$
\left\langle F_{n}(t), \psi\right\rangle_{\mathcal{V}^{*}, \mathcal{V}}=h_{n} \int_{\gamma_{2}}\left(\operatorname{Tr}_{2} \psi\right)(z) d S_{2}+S_{0}(t) \int_{\gamma_{4}}\left(\operatorname{Tr}_{4} \psi\right)(z) d S_{4} .
$$

Let $\left\{h_{n}\right\}$ be a sequence such that $h_{n} \rightarrow h$ and let $\left\{k_{n}\right\}$ be a sequence such that $k_{n} \rightarrow k$ uniformly in $z$, i.e., $k_{n} \rightarrow k$ in $C(\Omega)$. Since we know $h_{n} \rightarrow h$, this implies $F_{n} \rightarrow F$ in $L^{2}\left(0, T ; \mathcal{V}^{*}\right)$. From the previous section we know solutions depend continuously on the forcing function when $F_{n} \rightarrow F$ in $L^{2}\left(0, T ; \mathcal{V}^{*}\right)$. Thus without loss of generality we can suppress the dependence of $F$ on $h_{n}$ and take $F$ for $F_{n}$ in $(5.27)$ with $u_{n}\left(\cdot ; k_{n}, h_{n}\right)$ a weak solution of

$$
\left\{\begin{array}{l}
\dot{u}_{n}+A_{n} u_{n}=F \\
u_{n}(0)=u_{0}
\end{array}\right.
$$

where $A_{n}$ is defined as in (5.28). By (5.3) we know for $N_{1}$ sufficiently large there exist constants $\bar{K}_{L}>0$ and $\bar{K}_{U}<\infty$ such that

$$
k_{n}(z) \in\left[\bar{K}_{L}, \bar{K}_{U}\right]
$$




$$
k(z) \in\left[\bar{K}_{L}, \bar{K}_{U}\right]
$$

for all $z \in \Omega$ and all $n \geq N_{1}$. Since $h_{n} \rightarrow h$, for $N_{2}$ sufficiently large there exist constants $\bar{h}_{L}>0$ and $\bar{h}_{U}<\infty$ such that

$$
\begin{gathered}
h_{n} \in\left[\bar{h}_{L}, \bar{h}_{U}\right], \\
h \in\left[\bar{h}_{L}, \bar{h}_{U}\right]
\end{gathered}
$$

for all $n \geq N_{2}$. Without loss of generality, hereafter we assume all $n$ will satisfy $n \geq N=\max \left\{N_{1}, N_{2}\right\}$.

Note $\sigma_{n}$ is uniformly (in $\left.n\right) \mathcal{V}$-coercive satisfying $\sigma_{n}(\phi, \phi) \geq C_{1}\|\phi\|_{\mathcal{V}}^{2}$, where

$$
C_{1}=\min \left\{R_{U}^{-1} \bar{K}_{L}, \bar{h}_{L}\right\} M_{U}^{-1}
$$

and $C_{1}$ is independent of $n$.

Subtracting the weak form of (5.29) from (5.26) we obtain

$$
\left\langle\dot{u}(t)-\dot{u}_{n}(t), \psi\right\rangle_{\mathcal{V}^{*}, \mathcal{V}}+\sigma(u(t), \psi)-\sigma_{n}\left(u_{n}(t), \psi\right)=0
$$

for all $\psi \in \mathcal{V}$. Thus

$$
\begin{aligned}
\langle\dot{u}(t) & \left.-\dot{u}_{n}(t), \psi\right\rangle_{\mathcal{V}^{*}, \mathcal{V}}+\left\langle\frac{1}{g}\left(k \nabla u(t)-k_{n} \nabla u_{n}(t)\right), \nabla \psi\right\rangle_{\mathcal{H}} \\
& +\int_{\gamma_{2}}\left(h\left(\operatorname{Tr}_{2} u(t)\right)(z)-h_{n}\left(\operatorname{Tr}_{2} u_{n}(t)\right)(z)\right)\left(\operatorname{Tr}_{2} \psi\right)(z) d S_{2}=0
\end{aligned}
$$

for all $\psi \in \mathcal{V}$.

Adding and subtracting terms we see

$$
\begin{aligned}
\left\langle\dot{u}(t)-\dot{u}_{n}(t), \psi\right\rangle_{\mathcal{V}^{*}, \mathcal{V}} & +\left\langle\frac{1}{g}\left(k-k_{n}\right) \nabla u(t), \nabla \psi\right\rangle_{\mathcal{H}}+\left\langle\frac{1}{g} k_{n}\left(\nabla u(t)-\nabla u_{n}(t)\right), \psi\right\rangle_{\mathcal{H}} \\
+ & \left(h-h_{n}\right) \int_{\gamma_{2}}\left(\operatorname{Tr}_{2} u(t)\right)(z)\left(\operatorname{Tr}_{2} \psi\right)(z) d S_{2} \\
& +h_{n} \int_{\gamma_{2}}\left(\operatorname{Tr}_{2}\left(u(t)-u_{n}(t)\right)\right)(z)\left(\operatorname{Tr}_{2} \psi\right)(z) d S_{2}=0
\end{aligned}
$$

for all $\psi \in \mathcal{V}$. 
Fix $t \in[0, T]$ and let $\psi=u(t)-u_{n}(t)$ in the previous equation. Thus

$$
\begin{aligned}
\frac{1}{2} \frac{d}{d t}\{\| u(t)- & \left.u_{n}(t) \|_{\mathcal{H}}^{2}\right\}+\left\langle\frac{1}{g} k_{n}\left(\nabla u(t)-\nabla u_{n}(t)\right), \nabla u(t)-\nabla u_{n}(t)\right\rangle_{\mathcal{H}} \\
& +h_{n} \int_{\gamma_{2}}\left(\operatorname{Tr}_{2}\left(u(t)-u_{n}(t)\right)\right)(z)\left(\operatorname{Tr}_{2}\left(u(t)-u_{n}(t)\right)\right)(z) d S_{2} \\
= & \left\langle\frac{1}{g}\left(k_{n}-k\right) \nabla u(t), \nabla u(t)-\nabla u_{n}(t)\right\rangle_{\mathcal{H}} \\
& +\left(h_{n}-h\right) \int_{\gamma_{2}}\left(\operatorname{Tr}_{2} u(t)\right)(z)\left(\operatorname{Tr}_{2}\left(u(t)-u_{n}(t)\right)\right)(z) d S_{2}
\end{aligned}
$$

and so using the definition of $\sigma_{n}$ and integrating from 0 to $t$, for $t \in[0, T]$ we obtain

$$
\begin{aligned}
\| u(t)- & u_{n}(t) \|_{\mathcal{H}}^{2}+2 \int_{0}^{t} \sigma_{n}\left(u(\xi)-u_{n}(\xi), u(\xi)-u_{n}(\xi)\right) d \xi \\
= & 2 \int_{0}^{t}\left\langle\frac{1}{g}\left(k_{n}-k\right) \nabla u(\xi), \nabla u(\xi)-\nabla u_{n}(\xi)\right\rangle_{\mathcal{H}} d \xi \\
& +2 \int_{0}^{t}\left(h_{n}-h\right) \int_{\gamma_{2}}\left(\operatorname{Tr}_{2} u(\xi)\right)(z)\left(\operatorname{Tr}_{2}\left(u(\xi)-u_{n}(\xi)\right)\right)(z) d S_{2} d \xi
\end{aligned}
$$

Using the fact $\sigma_{n}$ is $\mathcal{V}$-coercive on the left side of the equation, and the CauchySchwartz inequality, the relation $2 a b \leq a^{2}+b^{2}$, and the equivalent form of the $\mathcal{V}$-norm generated by (5.5) on the right side, we see

$$
\begin{aligned}
\| u(t)- & u_{n}(t)\left\|_{\mathcal{H}}^{2}+2 C_{1} \int_{0}^{t}\right\| u(\xi)-u_{n}(\xi) \|_{\mathcal{V}}^{2} d \xi \\
\leq & \left.2 \int_{0}^{t}\left\|\frac{1}{g}\left(k_{n}-k\right) \nabla u(\xi)\right\|\right|_{\mathcal{H}}\left\|\nabla u(\xi)-\nabla u_{n}(\xi)\right\|_{\mathcal{H}} d \xi \\
& +2 \int_{0}^{t}\left|\left(h_{n}-h\right) \int_{\gamma_{2}}\left(\operatorname{Tr}_{2} u(\xi)\right)(z)\left(\operatorname{Tr}_{2}\left(u(\xi)-u_{n}(\xi)\right)\right)(z) d S_{2}\right| d \xi \\
\leq & \frac{2}{C_{1}} \int_{0}^{t} R_{L}^{-2}\left\|k_{n}-k\right\|_{\infty}^{2}\|\nabla u(\xi)\|_{\mathcal{H}}^{2} d \xi+\frac{C_{1}}{2} \int_{0}^{t}\left\|\nabla u(\xi)-\nabla u_{n}(\xi)\right\|_{\mathcal{H}}^{2} d \xi \\
& +\frac{2 M_{L}^{-1}}{C_{1}} \int_{0}^{t}\left|h_{n}-h\right|^{2} \int_{\gamma_{2}}\left|\left(\operatorname{Tr}_{2} u(\xi)\right)(z)\right|^{2} d S_{2} d \xi \\
& +\frac{C_{1}}{2 M_{L}^{-1}} \int_{0}^{t} \int_{\gamma_{2}}\left|\left(\operatorname{Tr}_{2}\left(u(\xi)-u_{n}(\xi)\right)\right)(z)\right|^{2} d S_{2} d \xi
\end{aligned}
$$




$$
\begin{aligned}
\leq & \frac{2}{C_{1} R_{L}^{2}}\left\|k_{n}-k\right\|_{\infty}^{2} \int_{0}^{t}\|u(\xi)\|_{\mathcal{V}}^{2} d \xi+\frac{C_{1}}{2} \int_{0}^{t}\left\|u(\xi)-u_{n}(\xi)\right\|_{\mathcal{V}}^{2} d \xi \\
& +\frac{2}{C_{1} M_{L}^{2}}\left|h_{n}-h\right|^{2} \int_{0}^{t}\|u(\xi)\|_{\mathcal{V}}^{2} d \xi+\frac{C_{1}}{2} \int_{0}^{t}\left\|u(\xi)-u_{n}(\xi)\right\|_{\mathcal{V}}^{2} d \xi
\end{aligned}
$$

where $\|\cdot\|_{\infty}$ is the $C(\Omega)$ norm. Combining like terms we see

$$
\begin{aligned}
\left\|u(t)-u_{n}(t)\right\|_{\mathcal{H}}^{2} & +C_{1} \int_{0}^{t}\left\|u(\xi)-u_{n}(\xi)\right\|_{\mathcal{V}}^{2} d \xi \\
& \leq\left(\frac{2}{C_{1} R_{L}^{2}}\left\|k_{n}-k\right\|_{\infty}^{2}+\frac{2}{C_{1} M_{L}^{2}}\left|h_{n}-h\right|^{2}\right) \int_{0}^{t}\|u(\xi)\|_{\mathcal{V}}^{2} d \xi
\end{aligned}
$$

If we define $G_{n}(T)$ as follows:

$$
G_{n}(T)=\left(\frac{2}{C_{1} R_{L}^{2}}\left\|k_{n}-k\right\|_{\infty}^{2}+\frac{2}{C_{1} M_{L}^{2}}\left|h_{n}-h\right|^{2}\right)\|u\|_{L^{2}(0, T ; \mathcal{V})}
$$

and recall $k_{n} \rightarrow k$ in $C(\Omega), h_{n} \rightarrow h$ and $u \in L^{2}(0, T ; \mathcal{V})$, we see $G_{n}(T) \rightarrow 0$. Thus

$$
\left\|u(t)-u_{n}(t)\right\|_{\mathcal{H}}^{2}+\frac{C_{1}}{2} \int_{0}^{t}\left\|u(\xi)-u_{n}(\xi)\right\|_{\mathcal{V}}^{2} d \xi \leq G_{n}(T)
$$

and so as $n \rightarrow \infty, u \rightarrow u_{n}$ in $C(0, T ; \mathcal{H})$ and in $L^{2}(0, T ; \mathcal{V})$. Thus the solution depends continuously on the parameters $k$ and $h$. We have proved the following theorem:

Theorem 5.5 The mapping $(k, h) \rightarrow u(\cdot ; k, h)$, where $u(\cdot ; k, h)$ is a solution to (5.26) is Lipschitz continuous from $C(\Omega) \times \mathbb{R}_{1}^{+}$to $C(0, T ; \mathcal{H}) \cap L^{2}(0, T ; \mathcal{V})$.

\subsubsection{Continuous Dependence on $\rho$ and $c_{p}$}

As before, let $g=\rho c_{p}$ and define a sequence $\left\{g_{n}\right\}$ such that $g_{n} \rightarrow g$ uniformly in $z$, i.e., in $C(\Omega)$. Thus we know by (5.2) for $N$ sufficiently large, there exist constants $\bar{R}_{L}>0$ and $\bar{R}_{U}<\infty$ such that for $\mathrm{N}$ sufficiently large,

$$
\begin{aligned}
g_{n}(z) & \in\left[\bar{R}_{L}, \bar{R}_{U}\right], \\
g(z) & \in\left[\bar{R}_{L}, \bar{R}_{U}\right]
\end{aligned}
$$

holds for all $n \geq N$ and all $z \in \Omega$. Without loss of generality, from hereafter we assume all $n$ will satisfy $n \geq N$. 
Let $\mathcal{H}_{n}=H^{1}(\Omega)$ with the weighted inner product $\langle\cdot, \cdot\rangle_{\mathcal{H}_{n}}=\left\langle g_{n} \cdot, \cdot\right\rangle_{L^{2}}$. Note the $\mathcal{H}_{n}$ norm is equivalent to the $\mathcal{H}$ norm uniformly in $n$ and there are finite, positive constants $J_{L}, J_{U}$ such that

$$
J_{L}\|\phi\|_{\mathcal{H}}^{2} \leq\|\phi\|_{\mathcal{H}_{n}}^{2} \leq J_{U}\|\phi\|_{\mathcal{H}}^{2}
$$

for all $n$. Define $\mathcal{V}_{n}=H^{1}(\Omega)$ with inner product

$$
\langle\phi, \psi\rangle_{\mathcal{V}_{n}}=\langle\nabla \phi, \nabla \psi\rangle_{\mathcal{H}_{n}}+\langle\phi, \psi\rangle_{L^{2}}
$$

which has equivalent representation

$$
\langle\phi, \psi\rangle_{\mathcal{v}_{n}} \cong\langle\nabla \phi, \nabla \psi\rangle_{\mathcal{H}_{n}}+\int_{\gamma_{2}} h\left(\operatorname{Tr}_{2} \phi\right)(z)\left(\operatorname{Tr}_{2} \psi\right)(z) d S_{2}
$$

by Theorem 5.1 .

Suppose $u(\cdot ; g)$ is a solution of

$$
\left\{\begin{array}{l}
\dot{u}+A u=F \\
u(0)=u_{0}
\end{array}\right.
$$

in $\mathcal{V}^{*}$ and suppose $u_{n}\left(\cdot ; g_{n}\right)$ is a solution of

$$
\left\{\begin{array}{l}
\dot{u}_{n}+A_{n} u_{n}=F_{n} \\
u_{n}(0)=u_{0}
\end{array}\right.
$$

in $\mathcal{V}_{n}^{*}$ where

$$
\left\langle A_{n} \phi, \psi\right\rangle_{\mathcal{V}_{n}^{*}, \mathcal{V}_{n}}=\sigma_{n}(\phi, \psi)=\left\langle\frac{1}{g_{n}} k \nabla \phi, \nabla \psi\right\rangle_{\mathcal{H}_{n}}+h \int_{\gamma_{2}}\left(\operatorname{Tr}_{2} \phi\right)(z)\left(\operatorname{Tr}_{2} \psi\right)(z) d S_{2}
$$

and

$$
\left\langle F_{n}(t) \phi, \psi\right\rangle_{\mathcal{V}_{n}^{*}, \mathcal{V}_{n}}=\langle F(t) \phi, \psi\rangle_{\mathcal{V}^{*}, \mathcal{V}}
$$

for all $\psi \in \mathcal{V}_{n} \cong \mathcal{V}$.

Subtracting the weak form of (5.33) from (5.32) we obtain

$$
\langle\dot{u}(t), \psi\rangle_{\mathcal{V}^{*}, \mathcal{V}}-\left\langle\dot{u}_{n}(t), \psi\right\rangle_{\mathcal{V}_{n}^{*}, \mathcal{V}_{n}}+\sigma(u(t), \psi)-\sigma_{n}\left(u_{n}(t), \psi\right)
$$




$$
=\langle F(t), \psi\rangle_{\mathcal{V}^{*}, \mathcal{V}}-\left\langle F_{n}(t), \psi\right\rangle_{\mathcal{V}_{n}^{*}, \mathcal{V}_{n}}
$$

for all $\psi \in \mathcal{V}$. Thus

$$
\begin{aligned}
\langle\dot{u}(t), \psi\rangle_{\mathcal{V}^{*}, \mathcal{V}}-\left\langle\dot{u}_{n}(t), \psi\right\rangle_{\mathcal{V}_{n}^{*}, \mathcal{V}_{n}}+\left\langle\frac{1}{g} k \nabla u(t), \nabla \psi\right\rangle_{\mathcal{H}} \\
+h \int_{\gamma_{2}}\left(\operatorname{Tr}_{2} u(t)\right)(z)\left(\operatorname{Tr}_{2} \psi\right)(z) d S_{2}-\left\langle\frac{1}{g_{n}} k \nabla u_{n}(t), \nabla \psi\right\rangle_{\mathcal{H}_{n}} \\
\quad-h \int_{\gamma_{2}}\left(\operatorname{Tr}_{2} u_{n}(t)\right)(z)\left(\operatorname{Tr}_{2} \psi\right)(z) d S_{2}=0
\end{aligned}
$$

and so

$$
\begin{aligned}
& \langle\dot{u}(t), \psi\rangle_{\mathcal{V}^{*}, \mathcal{V}}-\left\langle\dot{u}_{n}(t), \psi\right\rangle_{\mathcal{V}_{n}^{*}, \mathcal{V}_{n}}+\langle k \nabla u(t), \nabla \psi\rangle_{L^{2}} \\
& +h \int_{\gamma_{2}}\left(\operatorname{Tr}_{2} u(t)\right)(z)\left(\operatorname{Tr}_{2} \psi\right)(z) d S_{2}-\left\langle k \nabla u_{n}(t), \nabla \psi\right\rangle_{L^{2}} \\
& \quad-h \int_{\gamma_{2}}\left(\operatorname{Tr}_{2} u_{n}(t)\right)(z)\left(\operatorname{Tr}_{2} \psi\right)(z) d S_{2}=0
\end{aligned}
$$

for all $\psi \in \mathcal{V}$. Define $\tilde{\sigma}$ by

$$
\tilde{\sigma}(\phi, \psi)=\langle k \nabla \phi, \nabla \psi\rangle_{L^{2}}+h \int_{\gamma_{2}}\left(\operatorname{Tr}_{2} \phi\right)(z)\left(\operatorname{Tr}_{2} \psi\right)(z) d S_{2}
$$

and note $\tilde{\sigma}$ is $\mathcal{V}$-coercive satisfying $\tilde{\sigma}(\phi, \phi) \geq C_{2}\|\phi\|_{\mathcal{V}}$ where

$$
C_{2}=\min \left\{R_{U}^{-1} K_{L}, h\right\} M_{U}^{-1}
$$

Adding and subtracting terms we see

$$
\langle\dot{u}(t), \psi\rangle_{\mathcal{V}^{*}, \mathcal{V}}-\langle\dot{u}(t), \psi\rangle_{\mathcal{V}_{n}^{*}, \mathcal{V}_{n}}+\left\langle\dot{u}(t)-\dot{u}_{n}(t), \psi\right\rangle_{\mathcal{V}_{n}^{*}, \mathcal{V}_{n}}+\tilde{\sigma}\left(u(t)-u_{n}(t), \psi\right)=0
$$

Fix $t \in[0, T]$ and let $\psi=u(t)-u_{n}(t)$ in the previous equation. Thus

$$
\begin{aligned}
\frac{1}{2} \frac{d}{d t}\left\{\left\|u(t)-u_{n}(t)\right\|_{\mathcal{H}_{n}}^{2}\right\}+\tilde{\sigma}\left(u(t)-u_{n}(t), u(t)-u_{n}(t)\right) \\
=\left\langle\frac{g_{n}}{g} \dot{u}(t)-\dot{u}(t), u(t)-u_{n}(t)\right\rangle_{\mathcal{V}^{*}, \mathcal{V}}
\end{aligned}
$$


and so integrating from 0 to $t$ we obtain

$$
\begin{array}{r}
\left\|u(t)-u_{n}(t)\right\|_{\mathcal{H}_{n}}^{2}+2 \int_{0}^{t} \tilde{\sigma}\left(u(\xi)-u_{n}(\xi), u(\xi)-u_{n}(\xi)\right) d \xi \\
=2 \int_{0}^{t}\left\langle\left(\frac{g_{n}}{g}-1\right) \dot{u}(\xi), u(t)-u_{n}(t)\right\rangle_{\mathcal{V}^{*}, \mathcal{V}} d \xi .
\end{array}
$$

Since the $\mathcal{H}_{n}$ norm is equivalent to the $\mathcal{H}$ norm uniformly in $n$, using (5.31) we can rewrite this equation as

$$
\begin{array}{r}
J_{L}\left\|u(t)-u_{n}(t)\right\|_{\mathcal{H}}^{2}+2 \int_{0}^{t} \tilde{\sigma}\left(u(\xi)-u_{n}(\xi), u(\xi)-u_{n}(\xi)\right) d \xi \\
\leq 2 \int_{0}^{t}\left|\left\langle\left(\frac{g_{n}}{g}-1\right) \dot{u}(\xi), u(t)-u_{n}(t)\right\rangle_{\mathcal{V}^{*}, \mathcal{V}}\right| d \xi
\end{array}
$$

Using the fact that $\tilde{\sigma}$ is $\mathcal{V}$-coercive on the left side of the equation, and the Cauchy Schwartz inequality and the relation $2 a b \leq a^{2}+b^{2}$ on the right side, we see

$$
\begin{aligned}
J_{L} \| u(t)- & u_{n}(t)\left\|_{\mathcal{H}}^{2}+2 C_{2} \int_{0}^{t}\right\| u(\xi)-u_{n}(\xi) \|_{\mathcal{V}}^{2} d \xi \\
\leq & 2 \int_{0}^{t}\left\|\left(\frac{g_{n}}{g}-1\right) \dot{u}(\xi)\right\|_{\mathcal{V}^{*}}\left\|u(\xi)-u_{n}(\xi)\right\|_{\mathcal{V}} d \xi \\
\leq & \frac{1}{C_{2}} \int_{0}^{t}\left\|\left(\frac{g_{n}}{g}-1\right) \dot{u}(\xi)\right\|_{\mathcal{V}^{*}}^{2} d \xi+C_{2} \int_{0}^{t}\left\|u(\xi)-u_{n}(\xi)\right\|_{\mathcal{V}}^{2} d \xi
\end{aligned}
$$

Combining like terms we see

$$
J_{L}\left\|u(t)-u_{n}(t)\right\|_{\mathcal{H}}^{2}+C_{2} \int_{0}^{t}\left\|u(\xi)-u_{n}(\xi)\right\|_{\mathcal{V}}^{2} d \xi \leq \frac{1}{C_{2}} \int_{0}^{t}\left\|\frac{g_{n}}{g}-1\right\|_{\infty}^{2}\|\dot{u}(\xi)\|_{\mathcal{V}^{*}}^{2} d \xi
$$

where $\|\cdot\|_{\infty}$ is the $C(\Omega)$ norm. Let $G_{n}(T)$ be defined as follows:

$$
G_{n}(T)=\frac{1}{C_{2}}\left\|\frac{g_{n}}{g}-1\right\|_{\infty}^{2}\|\dot{u}\|_{L^{2}\left(0, T ; \mathfrak{\nu}^{*}\right)}
$$

and note $G_{n}(T) \rightarrow 0$ since $g_{n} \rightarrow g$ in $C(\Omega)$ and $\dot{u} \in L^{2}\left(0, T ; \mathcal{V}^{*}\right)$. Thus

$$
J_{L}|| u(t)-u_{n}(t)\left\|_{\mathcal{H}}^{2}+C_{2} \int_{0}^{t}\right\| u(\xi)-u_{n}(\xi) \|_{\mathcal{V}}^{2} d \xi \leq G_{n}(T)
$$

and so $u_{n} \rightarrow u$ in $C(0, T ; \mathcal{H})$ and in $L^{2}(0, T ; \mathcal{V})$. Thus the solution depends continuously on the parameters $\rho$ and $c_{p}$. We have proved the following theorem: 
Theorem 5.6 The mapping $g \rightarrow u(\cdot ; g)$, where $u(\cdot ; g)$ is a solution to (5.32) is continuous from $C(\Omega)$ to $C(0, T ; \mathcal{H}) \cap L^{2}(0, T ; \mathcal{V})$. 


\section{Chapter 6}

\section{Parameter Estimation for Two Dimensional Heat Transfer Problem}

In this chapter we present a parameter estimation formulation for our two dimensional model, as well as computational results. We begin by looking at estimating constant values for the thermal conductivity of the aluminum particles and of the silicone polymer. We then estimate these parameters as realizations of a random variable. A theoretical basis for this idea is presented, as is the formulation for our particular problem. Computational results from both approaches are presented.

\subsection{Preliminaries}

We start with the model from Section 3.2

$$
\begin{cases}g(z) \dot{u}(t, z)=\nabla \cdot(k(z) \nabla u(t, z)) & \\ \left.k(z) \frac{\partial u}{\partial n}(t, z)\right|_{\gamma_{4}}=S_{0}(t) & (\text { source }) \\ \left.k(z) \frac{\partial u}{\partial n}(t, z)\right|_{\gamma_{2}}=\left.h\left(T_{\infty}-u(t, z)\right)\right|_{\gamma_{2}} & (\text { sink }) \\ \left.k(z) \frac{\partial u}{\partial n}(t, z)\right|_{\gamma_{1}}=0 & \\ \left.k(z) \frac{\partial u}{\partial n}(t, z)\right|_{\gamma_{3}}=0 & \\ u(0, z)=\Upsilon(z) & \end{cases}
$$


for $z \in \Omega$ and $t \in[0, T]$. As in Chapter 5 we define $g(z)=\rho(z) c_{p}(z)$.

For $\left\{t_{i}\right\}_{i=1}^{n} \subset[0, T], T<\infty$, we can find (weak) solutions $u\left(t_{i}\right)$ to (6.1) for $1 \leq i \leq n<\infty$. From the solutions $\left\{u\left(t_{i}, z\right)\right\}_{i=1}^{n}$ we can compute the average temperature change between consecutive time steps at the heat sink interface (boundary $\gamma_{2}$ ). We define the average temperature change from $t_{i-1}$ to $t_{i}$ by

$$
T_{i}=\frac{1}{\left|\gamma_{2}\right|} \int_{\gamma_{2}} \operatorname{Tr}_{2}\left(u\left(t_{i}\right)-u\left(t_{i-1}\right)\right)(z) d S_{2}
$$

for $i=2, \ldots, n$. Recall $\operatorname{Tr}_{2}$ is the continuous trace operator from $H^{1}(\Omega) \rightarrow H^{\frac{1}{2}}\left(\gamma_{2}\right)$ defined by $\left(\operatorname{Tr}_{2} f\right)(z)=\left.f(z)\right|_{\gamma_{2}}$. We choose to look at the data this way in order to relate our model data to our experimental data from Section 2.3. Recall the Microflash gives the average voltage rise across the face of the test piece which we can then relate to the average temperature rise, $\Delta T$, as described in Section 2.3.

Before using the experimental data we have collected in our parameter estimation problem, we first use generated data for proof of concept. We generate data by solving (6.1) at the eight times in Table 3.2 with the parameter values from Table 3.1. We call these solutions $\hat{u}\left(t_{1}, z\right), \hat{u}\left(t_{2}, z\right), \ldots, \hat{u}\left(t_{8}, z\right)$. We define the vector of average temperature changes $\hat{T}=\left[\begin{array}{llll}\hat{T}_{2} & \hat{T}_{3} & \cdots & \hat{T}_{8}\end{array}\right]$ where

$$
\hat{T}_{j}=\frac{1}{\left|\gamma_{2}\right|} \int_{\gamma_{2}} \operatorname{Tr}_{2}\left(\hat{u}\left(t_{j}\right)-\hat{u}\left(t_{j-1}\right)\right)(z) d S_{2}
$$

for $2 \leq j \leq 8$. Furthermore, we define $T(q)$ to be the vector generated by the solution using the specified parameter (or parameters) $q$, which we will denote $u(t, z ; q)$, and (6.2), i.e.,

$$
T_{j}(q)=\frac{1}{\left|\gamma_{2}\right|} \int_{\gamma_{2}} \operatorname{Tr}_{2}\left(u\left(t_{j} ; q\right)-u\left(t_{j-1} ; q\right)\right)(z) d S_{2}
$$

for $2 \leq j \leq 8$ and $T(q)=\left[T_{2}(q) T_{3}(q) \cdots T_{8}(q)\right]$. We will assume any unspecified parameters are given by the values in Table 3.1 . 


\subsection{Estimating Constant Parameters}

We first try to estimate a constant value for the thermal conductivity of the aluminum particles that best matches our generated data. We define the cost function for this parameter estimation problem by

$$
J_{p}\left(\bar{q}_{p}, \hat{T}\right) \equiv J_{p}\left(\bar{q}_{p}\right) \equiv\left\|\hat{T}-T\left(\bar{q}_{p}\right)\right\|_{2}
$$

where

$$
\left\|\hat{T}-T\left(\bar{q}_{p}\right)\right\|_{2}=\left(\sum_{j=2}^{8}\left|\hat{T}_{j}-T_{j}\left(\bar{q}_{p}\right)\right|^{2}\right)^{\frac{1}{2}}
$$

and $\bar{q}_{p} \sim k_{p}$. Using Matlab's constrained minimization routine fmincon, we will try to find $\bar{q}_{p}^{*}$ which is a minimum for (6.3) over $Q \subset \mathbb{R}^{+}$. The function fmincon uses a Sequential Quadratic Programming method. The three main steps of this algorithm are the solving of a Quadratic Programming subproblem, the updating of the Hessian matrix of the Lagrangian solution, and the calculation of a merit function and line search. A complete description of this method can be found in [21].

Table 6.1 contains several initial guesses for $\bar{q}_{p}$, which we call $\bar{q}_{p_{0}}$, the cost associated with the initial guess, $J_{p}\left(\bar{q}_{p_{0}}\right)$, the solution generated by Matlab, $\bar{q}_{p}^{*}$, and the cost, $J_{p}\left(\bar{q}_{p}^{*}\right)$. Recall the data was generated using $k_{p}=217 \mathrm{~W} / \mathrm{mK}$.

\begin{tabular}{|c|c|c|c|}
\hline $\bar{q}_{p_{0}}$ & $J_{p}\left(\bar{q}_{p_{0}}\right)$ & $\bar{q}_{p}^{*}$ & $J_{p}\left(\bar{q}_{p}^{*}\right)$ \\
\hline \hline 100 & 0.00249265 & 100.001 & 0.00249265 \\
\hline 200 & 0.00908542 & 199.984 & 0.00905879 \\
\hline 500 & 0.0150171 & 720.563 & 0.0017473 \\
\hline
\end{tabular}

Table 6.1: Optimization results for estimating a constant value for $k_{p}$

Note for each of the three choices for $\bar{q}_{p_{0}}$, the optimal value selected by $\bar{q}_{p}^{*}$ is not close to the true value of $217 \mathrm{~W} / \mathrm{mK}$ and there is very little difference between $J_{p}\left(\bar{q}_{p_{0}}\right)$ and $J_{p}\left(\bar{q}_{p}^{*}\right)$. The small magnitude of $J_{p}\left(\bar{q}_{p_{0}}\right)$ and $J_{p}\left(\bar{q}_{p}^{*}\right)$ and the relatively small difference between the two suggests a possible reason for the inability to locate 
the correct value. However, it is unclear whether the problem is related to $k_{p}$ or to the formulation of the parameter estimation problem.

In comparison, we try to estimate the constant value for the thermal conductivity of the silicone polymer that best matches our generated data. In this case, we define the cost for the inverse problem by

$$
J_{s}\left(\bar{q}_{s}, \hat{T}\right) \equiv J_{s}\left(\bar{q}_{s}\right) \equiv\left\|\hat{T}-T\left(\bar{q}_{s}\right)\right\|_{2},
$$

where

$$
\left\|\hat{T}-T\left(\bar{q}_{s}\right)\right\|_{2}=\left(\sum_{j=2}^{8}\left|\hat{T}_{j}-T_{j}\left(\bar{q}_{s}\right)\right|^{2}\right)^{\frac{1}{2}}
$$

and $\bar{q}_{s} \sim k_{s}$. Again using Matlab, we try to find $\bar{q}_{s}^{*}$ which is a minimum for (6.4) over $Q \subset \mathbb{R}^{+}$. In Table 6.2 are several initial guesses for $\bar{q}_{s}$, which we will call $\bar{q}_{s_{0}}$ the cost associated with the initial guess, $J_{s}\left(\bar{q}_{s_{0}}\right)$, the solution generated by Matlab, $\bar{q}^{*}$, and the cost, $J_{s}\left(\bar{q}^{*}\right)$. Note for each of the choices for $\bar{q}_{s_{0}}$, the optimal solution is $\bar{q}_{s}^{*}=0.12$, which is the value for $k_{s}$ used in the generated data.

\begin{tabular}{|c|c|c|c|}
\hline $\bar{q}_{s_{0}}$ & $J_{s}\left(\bar{q}_{s_{0}}\right)$ & $\bar{q}_{s}^{*}$ & $J_{s}\left(\bar{q}_{s}^{*}\right)$ \\
\hline \hline 0.25 & 4.3188 & 0.12 & $9.874 \times 10^{-8}$ \\
\hline 0.05 & 4.6979 & 0.12 & $2.3140 \times 10^{-5}$ \\
\hline 0.6 & 4.6700 & 0.12 & $3.9795 \times 10^{-7}$ \\
\hline
\end{tabular}

Table 6.2: Optimization results for estimating a constant value for $k_{s}$

We appear to be successful when identifying the parameter $k_{s}$, but not the parameter $k_{p}$. Figures 6.1 and 6.2 give some insight into these results. In Figure 6.1, $J_{p}\left(\bar{q}_{p}\right)$ is plotted as a function of $\bar{q}_{p}$ for $\bar{q}_{p}=10,11,12, \ldots, 800$. Recall the true value for $k_{p}$ is $217 \mathrm{~W} / \mathrm{mK}$, so we expect $J_{p}(217)=0$, which is supported by the graph. However, the graph is not smooth and it is easy to see why the optimization routine was unable to find the correct value for $\bar{q}_{p}^{*}$ based on our initial guesses.

In contrast to Figure 6.1, Figure 6.2 depicts $J_{s}\left(\bar{q}_{s}\right)$ plotted as a function of $\bar{q}_{s}$ for $\bar{q}_{s}=0,0.001,0.002, \ldots, 1$. Recall the true value for $k_{s}$ is $0.12 \mathrm{~W} / \mathrm{mK}$ and in the graph 
we see $J_{p}(0.12)=0$ as expected. The graph of $J_{s}\left(\bar{q}_{s}\right)$ is smooth, and when compared to the graph of $J_{p}\left(\bar{q}_{p}\right)$ in Figure 6.1 is it understandable why our optimization routine had an easier time finding $\bar{q}_{s}^{*}$ over $\bar{q}_{p}^{*}$. Thus, we are led to conjecture that our parameter estimation problem formulation is appropriate. Furthermore, Figures 6.1 and $6.2 \mathrm{seem}$ to indicate our solution is more sensitive to $k_{s}$ than $k_{p}$. We address this further in Chapter 7. We now turn our attention to an alternative approach to parameter estimation.

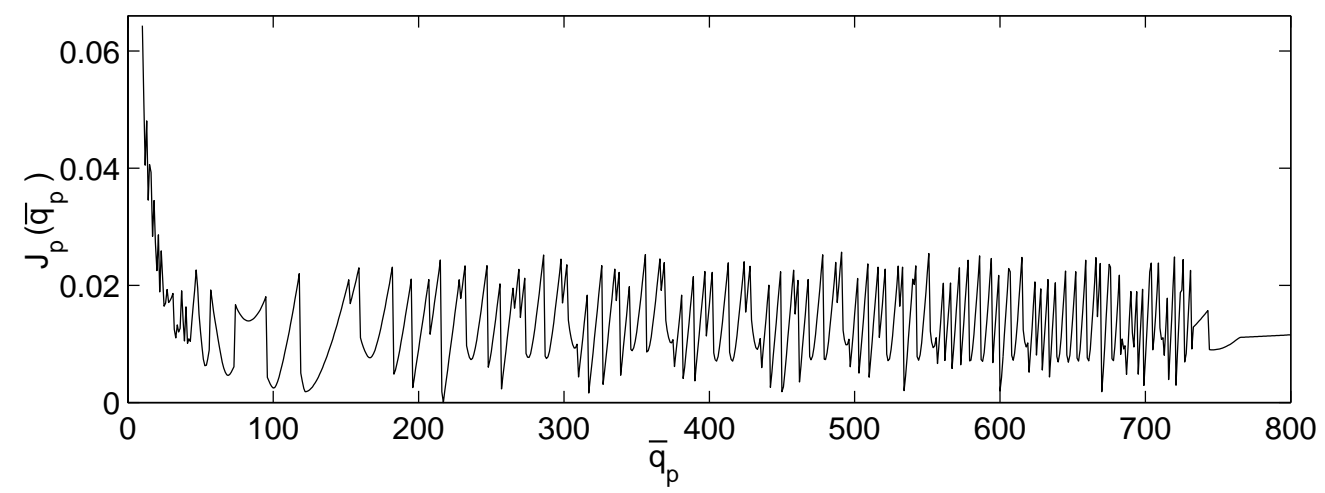

Figure 6.1: Cost $J_{p}\left(\bar{q}_{p}\right)$ for $\bar{q}_{p}=10,11,12, \ldots, 800$

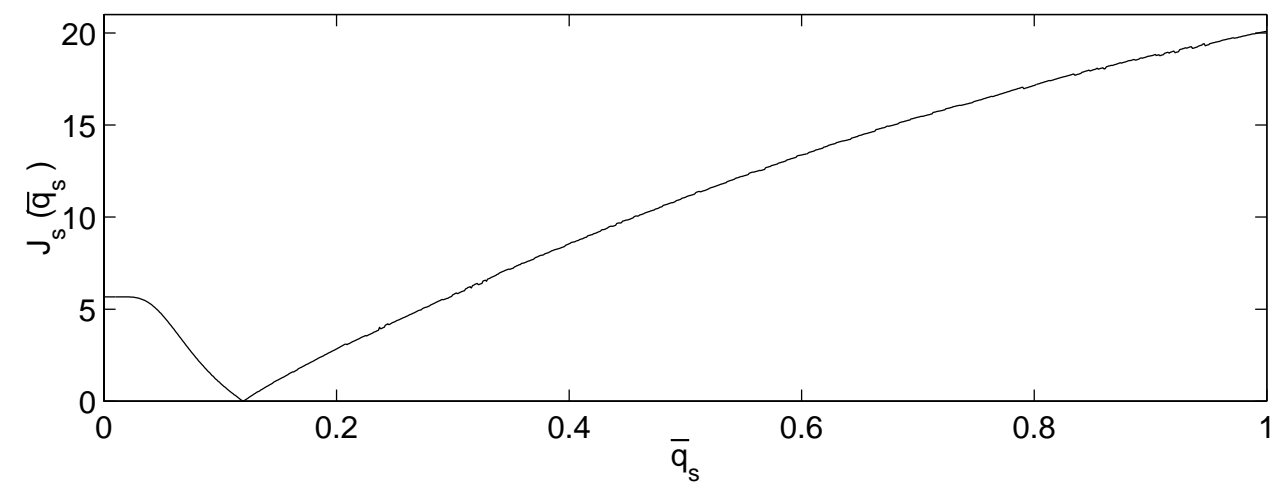

Figure 6.2: Cost $J_{s}\left(\bar{q}_{s}\right)$ for $\bar{q}_{s}=0,0.001,0.002, \ldots, 1$ 


\subsection{Estimating Parameters as Random Variable}

A standard deterministic inverse problem frequently encountered in both applied and theoretical literature can be abstractly stated as follows: Given a parameter dependent dynamical or algebraic system

$$
\mathcal{A}(q, u(q))=\mathcal{F}(q)
$$

with states $u(q)$, parameters $q$ and operators (differential or algebraic) $\mathcal{A}$, use observations (possibly incomplete) or data on the states to determine the best parameters $q^{*}$ in some admissible set $Q$ so that the solution of equation (6.5) for $q=q^{*}$ best describes the data. For such deterministic problems there is a large literature based on diverse formulations (least squares, equation error, etc.). For discussions of some of these see [5]. Once one has "solved" this (by no means trivial) deterministic problem, it is frequently important to know something about the reliability of the estimates. One approach entails attaching "error bars" to the estimated parameter values, much like one does in standard statistical analysis or in scientific computational analysis (using a priori bounds) with finite discretization techniques (finite difference, finite elements, etc) from numerical analysis. In essence we are asking for measurements of uncertainty related to our best estimates of parameters $q$. This uncertainty can come from a number of sources including: (i) intra-individual variability in data (in data collected from the same individual); (ii) inter-individual variability in data (aggregate data collected across a population of individuals); (iii) variability associated with measurement processes (as typically embodied in repeated measurement Bayesian paradigms and other approaches to regression analysis $[12,18]$ ); (iv) variability associated with either the optimization algorithm or the simulation algorithm or both in the inverse problem methodology. In any of these cases we are thus lead in a completely natural way to stochastic or probabilistic aspects of estimates from a deterministic problem solved with deterministic algorithms.

We offer here ideas for one approach (given in [1]) to treatment of variability in 
parameter estimation techniques. The approach is based on viewing multiple observations $\left\{\hat{u}_{j}\right\}_{j=1}^{N}$ of the state in (6.5) as observations corresponding to a set of realizations $\left\{q_{j}\right\}_{j=1}^{N}$ of the parameter $q$ which is now thought of as a specific (albeit unknown) random variable with probability distribution $P$ on $Q$. The system (6.5) is accordingly reformulated in terms of the state $u=u(P)$ depending on the probability distribution $P$. The observations $\left\{\hat{u}_{j}\right\}$ can be averaged so that $\hat{u}=\frac{1}{N} \sum_{j=1}^{N} \hat{u}_{j}$ is an observation for $u(P)$ and one can then attempt to estimate a best distribution $P^{*}$ to fit this data $\hat{u}$, for example in some type of least squares fit. Once one obtains $P^{*}$, its mean $\mu$ and variance $\sigma^{2}$ can be used as a best parameter estimate and measure of reliability, respectively.

In the sections below we summarize the results in [1] in a concrete example using nonlinear parameter dependent ordinary differential equations for the system (6.5). We present a precise formulation of this conceptual approach, show that fundamental results from probability theory can be used to develop well-posedness results (existence, continuous dependence, and method stability) along with approximation ideas that are computationally tractable. In Section 6.4 we apply these results to our parameter estimation problem, estimating the thermal conductivity parameters in our two dimensional model. The data collection procedure described in Section 2.2 shows we will have repeated measurements. Furthermore, the nature of the composite silicone, as well as the potential error in data collection, show we will have variability in our data, both inter-individual variability and intra-individual variability. Thus we believe this method is very appropriate. We refer the reader to [1] for further information and an additional application of the methodology.

\subsubsection{Parameter Estimation in Nonlinear Systems}

To demonstrate our ideas, we will examine the estimation of constant parameters in a system of ordinary differential equations. These ideas can be easily extended to many systems of interest in applications, including systems of partial differential equations with unknown functional parameters (e.g., time and/or spatially dependent 
coefficients).

A typical estimation problem employs observations $\hat{x}=\left\{\hat{x}_{i}\right\}_{i=1}^{n}$ for $x\left(t_{i}\right), i=$ $1,2, \ldots, n$, to estimate parameters $q \in \mathbb{R}^{m}$ in the vector dynamical system

$$
\dot{x}(t)=f(t, x(t), q) .
$$

Often a least squares formulation is used to find a best parameter value $q^{*}$ in some admissible parameter set $Q \subset \mathbb{R}^{m}$. In other words, we attempt to find $q^{*} \in Q$ which is a minimum for

$$
J(q, \hat{x})=\sum_{i=1}^{n}\left|x\left(t_{i} ; q\right)-\hat{x}_{i}\right|^{2}
$$

over $q \in Q$ where $x\left(t_{i} ; q\right)$ is a solution of (6.6) for a given $q \in Q$. We assume throughout that $f$ is sufficiently regular so that $q \rightarrow x(t ; q)$ is continuous on $Q$ for each $t$.

To introduce uncertainty, we view the parameters as realizations for a random variable and use the data to estimate the probability distribution function (PDF) for this random variable. Specifically, let $\mathcal{P}(Q)$ denote the set of probability distributions on $Q$ and treat the data $\left\{\hat{x}_{i}\right\}$ as observations for the expected value

$$
\mathcal{E}\left[x\left(t_{i} ; q\right) \mid P\right]=\int_{Q} x\left(t_{i} ; q\right) d P(q)
$$

for a given PDF $P \in \mathcal{P}(Q)$. Note that if $P$ is a discrete PDF with atoms $\left\{q_{j}\right\}_{j=1}^{M} \subset Q$ and associated probabilities $\left\{p_{j}\right\}, p_{j} \geq 0, \sum_{j=1}^{M} p_{j}=1$, then (6.7) can be written

$$
\int_{Q} x\left(t_{i} ; q\right) d P(q)=\sum_{j=1}^{M} x\left(t_{i} ; q_{j}\right) p_{j} .
$$

Regardless of the form of $P$, the least squares estimation problem can be described as finding $P^{*} \in \mathcal{P}(Q)$ to minimize

$$
J(P)=\sum_{i=1}^{n}\left|\mathcal{E}\left[x\left(t_{i} ; q\right) \mid P\right]-\hat{x}_{i}\right|^{2}
$$

over $P \in \mathcal{P}(Q)$. To develop theoretical and computational results for this problem, it is necessary to have a topology on $\mathcal{P}(Q)$, continuity of the function $P \rightarrow J(P)$ in this 
topology, compatible compactness results, and some approximation results leading to implementable computational algorithms. In order to address these issues we will introduce the Prohorov metric and summarize some results from Billingsley [10].

\subsubsection{The Prohorov Metric in the Space of Probability Dis- tributions}

Let $\mathcal{P}(Q)$ be the set of probability measures on the Borel subsets of $\mathrm{Q}$, where $\mathrm{Q}$ is any complete metric space with metric $d$. For any closed subset $F \subset Q$ and $\epsilon>0$, we define an $\epsilon$-neighborhood of $\mathrm{F}$ by

$$
F^{\epsilon}=\{q \in Q: d(\tilde{q}, q)<\epsilon, \tilde{q} \in F\}
$$

We then define $\rho: \mathcal{P}(Q) \times \mathcal{P}(Q) \rightarrow \mathbb{R}^{+}$by

$$
\rho\left(P_{1}, P_{2}\right) \equiv \inf \left\{\epsilon>0: P_{1}[F] \leq P_{2}\left[F^{\epsilon}\right]+\epsilon, F \text { closed, } F \subset Q\right\}
$$

The following properties of $\rho$ are well-known:

(a) $\rho$ is a metric (called the Prohorov metric) on $\mathcal{P}(Q)$;

(b) $(\mathcal{P}(Q), \rho)$ is a complete metric space;

(c) if $Q$ is compact, then $(\mathcal{P}(Q), \rho)$ is a compact metric space.

We would like to understand convergence of $P_{k} \rightarrow P$ in the $\rho$ metric. Unfortunately, the Prohorov metric is neither intuitive nor easy to use directly. However, it is well known that if $(Q, d)$ is a complete metric space and $(\mathcal{P}(Q), \rho)$ is defined as above, then for $P_{k}, P \in \mathcal{P}(Q)$, the following convergence statements are equivalent:

(i) $\rho\left(P_{k}, P\right) \rightarrow 0$;

(ii) $\int_{Q} f d P_{k}(q) \rightarrow \int_{Q} f d P(q)$ for all bounded, uniformly continuous $f: Q \rightarrow \mathbb{R}^{1}$;

(iii) $P_{k}[A] \rightarrow P[A]$ for all Borel sets $A \subset Q$ with $P[\partial A]=0$, where $\partial A$ denotes the boundary of $A$. 
The equivalence of (i) and (iii) reveals that convergence in the $\rho$ metric is equivalent to convergence in distribution. Moreover, if we consider $\mathcal{P}(Q)$ as a set of bounded linear functionals on the space $C_{B}(Q)$ of bounded continuous functions on $Q$ with supremum norm, and hence as a subset of the space $C_{B}^{*}(Q)$ of all bounded linear functionals on $C_{B}(Q)$, then (i) and (ii) imply that convergence in the $\rho$ topology is equivalent to weak* convergence in $\mathcal{P}(Q)$. For our discussions, we will make critical use of the equivalence between $\rho\left(P_{k}, P\right) \rightarrow 0$ and

$$
\int_{Q} x(t ; q) d P_{k}(q) \rightarrow \int_{Q} x(t ; q) d P(q),
$$

which in turn is the same as

$$
\mathcal{E}\left[x(t ; q) \mid P_{k}\right] \rightarrow \mathcal{E}[x(t ; q) \mid P]
$$

This convergence is needed to establish continuity in the $\rho$ topology of the map

$$
P \rightarrow J(P)=\sum_{i=1}^{n}\left|\mathcal{E}\left[x\left(t_{i} ; q\right) \mid P\right]-\hat{x}_{i}\right|^{2}
$$

Continuity of this map, along with the compactness of $Q$, which guarantees compactness of $\mathcal{P}(Q)$ in the $\rho$ metric, is sufficient to establish existence of a solution to the problem of minimizing (6.8) over $\mathcal{P}(Q)$.

If we assume existence questions are answered, and turn to the task of characterizing and/or finding minimizers, we note that $\mathcal{P}(Q)$ with the $\rho$ metric is in general an infinite dimensional space. Thus, to address computational issues one must consider approximation ideas. To do this we first prove a density theorem that will be useful in establishing continuous dependence of estimates on data as well as in constructing approximation schemes.

There are numerous topologies on $\mathcal{P}(Q)$. If we define a $\mathcal{W}$-neighborhood of $P$ as $N_{\delta}(P)=\left\{P_{1} \in \mathcal{P}(Q): P_{1}\left(F_{i}\right)<P\left(F_{i}\right)+\delta, i=1, \ldots, k, F_{i}\right.$ closed, $\left.F_{i} \in \mathbb{S}\right\}$ for a given $\delta>0$ and finite set $\left\{F_{i}\right\}_{i=1}^{k}$ where $\mathbb{S}$ is the class of all Borel subsets of $Q$, this induces a topology on $\mathcal{P}(Q)$ which is equivalent to the topology of weak convergence, $\mathcal{W}$ (see $[10$, p. 236]). We can also define a $\rho$-neighborhood of $P$ by 
$\bar{N}_{\epsilon}(P)=\left\{P_{1} \in \mathcal{P}(Q): \rho\left(P, P_{1}\right)<\epsilon\right\}$ for a given $\epsilon>0$. If $Q$ is a separable space, the $\mathcal{W}$ topology is equivalent to the topology induced by the $\rho$-neighborhoods (see $[10$, pp. 238,239]). We will be using the equivalence of these two topologies in the proof of Theorem 6.1. Here $\mathbb{N}^{+}$are the positive integers, $\mathcal{R}$ are the rational numbers, and $\delta_{q_{j}}$ is the Dirac measure with atom at $q_{j}$.

For further information on the Prohorov metric and results relating to the convergence of probability measures, see [14].

Theorem 6.1 (Banks/Bihari [1]) Let $Q$ be a complete, separable metric space with metric $d, \mathbb{S}$ be the class of all Borel subsets of $Q$ and $\mathcal{P}(Q)$ be the space of probability measures on $(Q, \mathbb{S})$. Let $Q_{0}=\left\{q_{j}\right\}_{j=1}^{\infty}$ be a countable, dense subset of $Q$. Then the set of $P \in \mathcal{P}(Q)$ such that $P$ has finite support in $Q_{0}$ and rational masses is dense in $\mathcal{P}(Q)$ in the $\rho$ metric. That is,

$\mathcal{P}_{0}(Q) \equiv\left\{P \in \mathcal{P}(Q): P=\sum_{j=1}^{k} p_{j} \delta_{q_{j}}, k \in \mathbb{N}^{+}, q_{j} \in Q_{0}, p_{j} \in \mathcal{R}, p_{j} \geq 0, \sum_{j=1}^{k} p_{j}=1\right\}$

is dense in $\mathcal{P}(Q)$ relative to $\rho$.

Proof: Let $\epsilon>0$ and let $P \in \mathcal{P}(Q)$. Let $\bar{N}_{\epsilon}(P)$ be a $\rho$-neighborhood of $P$. Since $Q$ is separable, the $\mathcal{W}$ and $\rho$ topologies are equivalent. Thus there is a $\delta>0$ such that $N_{\delta}(P) \subseteq \bar{N}_{\epsilon}(P)$, where $N_{\delta}(P)$ is a $\mathcal{W}$-neighborhood of $\mathrm{P}$ of the form described above with closed sets $F_{1}, \ldots, F_{k}$.

Let $\left\{B_{i}\right\}_{i=1}^{M}$ be the partition of $\bigcup_{i=1}^{k} F_{i} \subset Q$ generated by the closed sets $F_{1}, \ldots, F_{k}$. We will assume each $B_{i}$ is non-empty and so $M<\infty$. Since $Q_{0}$ is a dense subset of $Q, B_{i} \cap Q_{0} \neq \emptyset$, for $i=1, \ldots, M$.

For $i=1, \ldots, M$, select a point $x_{i} \in B_{i} \cap Q_{0}$. At each point, $x_{i}$, place a mass, $b_{i}$, which satisfies the following three conditions: i) $b_{i} \in \mathcal{R}$, ii) $0 \leq b_{i} \leq P\left(B_{i}\right)$, and iii) $\left|P\left(B_{i}\right)-b_{i}\right|<\frac{\delta}{2 M}$.

Now if $\cup_{i=1}^{k} F_{i} \neq Q$, select a point $x_{M+1}$ so that $x_{M+1} \in Q_{0} \cap\left(\cup_{i=1}^{k} F_{i}\right)^{C}$. If $\cup_{i=1}^{k} F_{i}=Q$, choose $x_{M+1}$ so that $x_{M+1} \in Q_{0} \backslash\left(\left\{x_{i}\right\}_{i=1}^{M}\right)$. In either case, place at $x_{M+1}$ a mass $b_{M+1} \equiv 1-\sum_{i=1}^{M} b_{i}$. Note that $b_{M+1} \in \mathcal{R}$ and $0 \leq b_{M+1} \leq 1$. 
Define $P^{*}=\sum_{i=1}^{M+1} b_{i} \delta_{q_{i}}$. Then $P^{*}(Q)=\sum_{i=1}^{M+1} b_{i}=1$, and $0 \leq P^{*}(A) \leq 1$ for all $A \in \mathbb{S}$. Thus $P^{*} \in \mathcal{P}_{0}(Q)$.

Define $K_{i}=\left\{j: F_{i} \cap B_{j} \neq \emptyset, 1 \leq j \leq M\right\}$. Note the set $K_{i}$ has at most $M$ indices. Now suppose $\cup_{i=1}^{k} F_{i} \neq Q$. Then for any $F_{i}$,

$$
\begin{aligned}
\left|P^{*}\left(F_{i}\right)-P\left(F_{i}\right)\right| & =\left|P^{*}\left(\bigcup_{j \in K_{i}} B_{j}\right)-P\left(\bigcup_{j \in K_{i}} B_{j}\right)\right| \\
& =\left|\sum_{j \in K_{i}}\left[P^{*}\left(B_{j}\right)-P\left(B_{j}\right)\right]\right| \\
& =\left|\sum_{j \in K_{i}}\left[b_{j}-P\left(B_{j}\right)\right]\right| \\
& <\sum_{j \in K_{i}} \frac{\delta}{2 M} \\
& <\delta .
\end{aligned}
$$

Now suppose $\cup_{i=1}^{k} F_{i}=Q$. If $x_{M+1} \notin F_{i}$, the above argument shows $\mid P^{*}\left(F_{i}\right)-$ $P\left(F_{i}\right) \mid<\delta$. If $x_{M+1} \in F_{i}$, then

$$
\begin{aligned}
\left|P^{*}\left(F_{i}\right)-P\left(F_{i}\right)\right| & =\left|P^{*}\left(\bigcup_{j \in K_{i}} B_{j}\right)-P\left(\bigcup_{j \in K_{i}} B_{j}\right)\right| \\
& =\left|\sum_{j \in K_{i}} P^{*}\left(B_{j}\right)-\sum_{j \in K_{i}} P\left(B_{j}\right)\right| \\
& =\left|\sum_{j \in K_{i}} b_{j}+b_{M+1}-\sum_{j \in K_{i}} P\left(B_{j}\right)\right| \\
& =\left|\sum_{j \in K_{i}}\left[b_{j}-P\left(B_{j}\right)\right]+\left[1-\sum_{j=1}^{M} b_{j}\right]\right|
\end{aligned}
$$




$$
\begin{aligned}
& =\left|\sum_{j \in K_{i}}\left[b_{j}-P\left(B_{j}\right)\right]+\sum_{j=1}^{M}\left[P\left(B_{j}\right)-b_{j}\right]\right| \\
& <\sum_{j \in K_{i}} \frac{\delta}{2 M}+\sum_{j=1}^{M} \frac{\delta}{2 M} \\
& \leq \delta .
\end{aligned}
$$

Thus for all $F_{i}, P^{*}\left(F_{i}\right)<P\left(F_{i}\right)+\delta$, so $P^{*} \in N_{\delta}(P)$.

Since $N_{\delta}(P) \subseteq \bar{N}_{\epsilon}(P), P^{*} \in \bar{N}_{\epsilon}(P)$. By construction $P^{*} \in \mathcal{P}_{0}(Q)$, so $\mathcal{P}_{0}(Q)$ is dense in $\mathcal{P}(Q)$ relative to $\rho$.

Theorem 6.1 can be used as a basis for defining a class of approximating sets to be used in tractable computational methods for the inverse problems defined in Section 6.3.1. First define

$$
Q_{d}=\bigcup_{M=1}^{\infty} Q_{M}
$$

where $Q_{M}=\left\{q_{j}^{M}\right\}_{j=1}^{M}, M=1,2, \ldots$, are chosen so that $Q_{d}$ is dense in $Q$. Note that $Q_{d}$ is countable. For each positive integer $M$ let

$$
\mathcal{P}^{M}(Q)=\left\{P \in \mathcal{P}(Q): P=\sum_{j=1}^{M} p_{j} \delta_{q_{j}^{M}}, q_{j}^{M} \in Q_{M}, p_{j} \in \mathcal{R}, p_{j} \geq 0, \sum_{j=1}^{M} p_{j}=1\right\} .
$$

If we then define

$$
\mathcal{P}_{d}(Q)=\bigcup_{M=1}^{\infty} \mathcal{P}^{M}(Q),
$$

then by Theorem 6.1 we know $\mathcal{P}_{d}(Q)$ is dense in $\mathcal{P}(Q)$, and so we can approximate any element $P \in \mathcal{P}(Q)$ by a sequence $\left\{P_{M_{j}}\right\}, P_{M_{j}} \in \mathcal{P}^{M_{j}}(Q)$, such that $\rho\left(P_{M_{j}}, P\right) \rightarrow 0$ as $M_{j} \rightarrow \infty$. 


\subsubsection{Stability of the Inverse Problem}

We now turn to the study of the inverse problem. We return to our original problem of finding a solution to

$$
\min _{P \in \mathcal{P}(Q)} J(P, \hat{x})=\sum_{i=1}^{n}\left|\mathcal{E}\left[x\left(t_{i} ; q\right) \mid P\right]-\hat{x}_{i}\right|^{2} .
$$

Given data $\hat{x}^{k}$ and $\hat{x}$ such that $\hat{x}^{k} \rightarrow \hat{x}$ as $k \rightarrow \infty$ and corresponding solutions $P^{*}\left(\hat{x}^{k}\right)$ and $P^{*}(\hat{x})$ (which in general are sets because there is not necessarily a unique minimizer of (6.12)), we say the problem is continuously dependent on the data (or stable) if $\operatorname{dist}\left(P^{*}\left(\hat{x}^{k}\right), P^{*}(\hat{x})\right) \rightarrow 0$ as $k \rightarrow \infty$ (see [5, 7] for detailed discussions and motivation). Here $\operatorname{dist}(A, B)$ is the usual Hausdorff distance between sets $A$ and $B$ (see [17, p. 123]) given by $\operatorname{dist}(A, B)=\inf \left\{\rho\left(P_{1}, P_{2}\right): P_{1} \in A, P_{2} \in B\right\}$.

We now define a series of approximate problems. Let $\mathcal{P}^{M}(Q)$ be defined as in (6.10) where $Q_{d}$ is a countable dense subset of $\mathrm{Q}$ as defined in (6.9) with $Q_{M}=\left\{q_{j}^{M}\right\}$. We define the approximate problem as finding a solution to

$$
\min _{P_{M} \in \mathcal{P}^{M}(Q)} J\left(P_{M}, \hat{x}\right)=\sum_{i=1}^{n}\left|\mathcal{E}\left[x\left(t_{i} ; q\right) \mid P_{M}\right]-\hat{x}_{i}\right|^{2} .
$$

Let $P_{M}^{*}(\hat{x})$ denote the set of solutions for a given $\hat{x}$. The problems are method stable (again, see [5, 7] for further discussions) if for any data $\hat{x}^{k}$ and $\hat{x}$ such that $\hat{x}^{k} \rightarrow \hat{x}$ as $k \rightarrow \infty$ we have $\operatorname{dist}\left(P_{M}^{*}\left(\hat{x}^{k}\right), P^{*}(\hat{x})\right) \rightarrow 0$ as $k \rightarrow \infty$ and $M \rightarrow \infty$. Note that this is equivalent to requiring $\operatorname{dist}\left(P_{M}^{*}\left(\hat{x}^{k}\right), P_{M}^{*}(\hat{x})\right) \rightarrow 0$ as $k \rightarrow \infty$ uniformly in $M$.

Theorem 6.2 (Banks/Bihari [1]) Let $Q$ be a compact metric space and assume solutions $x(t ; q)$ of $(6.6)$ are continuous in $q$ on $Q$. Let $\mathcal{P}(Q)$ be the set of all probability measures on $Q$ and let $Q_{d}$ be a countable dense subset of $Q$ as defined in (6.9) with $Q_{M}=\left\{q_{j}^{M}\right\}_{j=1}^{M}$. Define $\mathcal{P}_{d}(Q)$ as in (6.11) where $\mathcal{P}^{M}(Q)$ is defined as in (6.10). Suppose $P_{M}^{*}\left(\hat{x}^{k}\right)$ is the set of minimizers for $J(P)$ over $P \in \mathcal{P}^{M}(Q)$ corresponding to the data $\left\{\hat{x}^{k}\right\}$ and $P^{*}(\hat{x})$ is the set of minimizers over $P \in \mathcal{P}(Q)$ corresponding to $\{\hat{x}\}$ where $\hat{x}^{k}, \hat{x} \in \mathbb{R}^{n}$ are the observed data such that $\hat{x}^{k} \rightarrow \hat{x}$. 
Then $\operatorname{dist}\left(P_{M}^{*}\left(\hat{x}^{k}\right), P^{*}(\hat{x})\right) \rightarrow 0$ as $M \rightarrow \infty$ and $\hat{x}^{k} \rightarrow \hat{x}$. Thus the solutions depend continuously on the data and the approximate problems are method stable.

Proof: Since $Q$ is a compact, separable metric space, $Q_{d}$ is dense in $Q$ and $\mathcal{P}_{d}(Q)$ is the space of all probability measures with finite support in $Q_{d}$ and rational masses, it follows from Theorem 6.1 that $\mathcal{P}_{d}(Q)$ is a dense subset of $\mathcal{P}(Q)$.

Since $Q$ is compact, $(\mathcal{P}(Q), \rho)$ is compact, where $\rho$ is the Prohorov metric. Since $q \rightarrow x(t, q)$ is continuous from $Q$ to $\mathbb{R}^{n}$, whenever $P_{M} \rightarrow P$ in $\mathcal{P}(Q)$ we have that

$$
\begin{aligned}
\lim _{M \rightarrow \infty} J\left(P_{M}\right) & =\lim _{M \rightarrow \infty} \sum_{i=1}^{n}\left|\mathcal{E}\left[x\left(t_{i} ; q\right) \mid P_{M}\right]-\hat{x}_{i}\right|^{2} \\
& =\sum_{i=1}^{n}\left|\mathcal{E}\left[x\left(t_{i} ; q\right) \mid P\right]-\hat{x}_{i}\right|^{2} \\
& =J(P) .
\end{aligned}
$$

Since $\mathcal{P}^{M}(Q)$ is a closed subset of $\mathcal{P}(Q), \mathcal{P}^{M}(Q)$ is compact in the $\rho$ topology. Moreover, $J$ is a continuous function on the compact set $\mathcal{P}^{M}(Q)$ and thus for a given set of observations $\hat{x}^{k}=\left\{\hat{x}_{i}^{k}\right\}_{i=1}^{n} \in \mathbb{R}^{n}$, there exists a (not necessarily unique) minimizer $P_{M}^{* k}$ which is a solution to the problem of minimizing $J^{k}(P)$ over $P \in \mathcal{P}^{M}(Q)$ where

$$
J^{k}(P) \equiv J\left(P, \hat{x}^{k}\right) \equiv \sum_{i=1}^{n}\left|\mathcal{E}\left[x\left(t_{i} ; q\right) \mid P\right]-\hat{x}_{i}^{k}\right|^{2} .
$$

Let $\left\{\hat{x}^{k}\right\}$ be a sequence that converges to some arbitrary $\hat{x} \in \mathbb{R}^{n}$. Let $P_{M}^{*}\left(\hat{x}^{k}\right)$ denote the set of minimizers of $J^{k}(P)$ over $\mathcal{P}^{M}(Q)$. For $k=1,2, \ldots$ and $M=1,2, \ldots$, let $\left\{P_{M}^{* k}\right\}, P_{M}^{* k} \in P_{M}^{*}\left(\hat{x}^{k}\right)$, be any sequence of minimizers in $\mathcal{P}(Q)$. By compactness there exists a convergent subsequence $\left\{P_{M_{\ell}}^{* k_{j}}\right\}$ such that

$$
\lim _{M_{\ell}, k_{j} \rightarrow \infty} P_{M_{\ell}}^{* k_{j}}=\tilde{P} \in \mathcal{P}(Q)
$$

in the $\rho$ metric.

First note that for any $P_{M_{\ell}} \in \mathcal{P}^{M_{\ell}}(Q)$

$$
J^{k_{j}}\left(P_{M_{\ell}}^{* k_{j}}\right) \leq J^{k_{j}}\left(P_{M_{\ell}}\right)
$$


Then by definition of $J^{k_{j}}\left(P_{M_{\ell}}^{* k_{j}}\right),(6.14)$, and (6.15)

$$
\begin{aligned}
\lim _{k_{j}, M_{\ell} \rightarrow \infty} J^{k_{j}}\left(P_{M_{\ell}}^{* k_{j}}\right) & =\lim _{k_{j}, M_{\ell} \rightarrow \infty} \sum_{i=1}^{n}\left|\mathcal{E}\left[x\left(t_{i} ; q\right) \mid P_{M_{\ell}}^{* k_{j}}\right]-\hat{x}_{i}^{k_{j}}\right|^{2} \\
& =\sum_{i=1}^{n}\left|\mathcal{E}\left[x\left(t_{i} ; q\right) \mid \tilde{P}\right]-\hat{x}_{i}\right|^{2} \\
& =J(\tilde{P}) .
\end{aligned}
$$

Let $P$ be any element of $\mathcal{P}(Q)$. Since $\mathcal{P}_{d}(Q)$ is dense in $\mathcal{P}(Q)$ we can find a sequence $\left\{P_{M_{\ell}}\right\}, P_{M_{\ell}} \in \mathcal{P}^{M_{\ell}}(Q)$, so that $P_{M_{\ell}} \rightarrow P$ in the $\rho$ metric as $M_{\ell} \rightarrow \infty$. Then by definition of $J^{k_{j}}\left(P_{M_{\ell}}\right)$, and (6.14)

$$
\begin{aligned}
\lim _{k_{j}, M_{\ell} \rightarrow \infty} J^{k_{j}}\left(P_{M_{\ell}}\right) & =\lim _{k_{j}, M_{\ell} \rightarrow \infty} \sum_{i=1}^{n}\left|\mathcal{E}\left[x\left(t_{i} ; q\right) \mid P_{M_{\ell}}\right]-\hat{x}_{i}^{k_{j}}\right|^{2} \\
& =\sum_{i=1}^{n}\left|\mathcal{E}\left[x\left(t_{i} ; q\right) \mid P\right]-\hat{x}_{i}\right|^{2} \\
& =J(P) .
\end{aligned}
$$

So (6.16), (6.17), and (6.18) gives

$$
J(\tilde{P}) \leq J(P)
$$

for any $P \in \mathcal{P}(Q)$. Hence $\tilde{P}$ is a minimizer of $J(P)$ over $P \in \mathcal{P}(Q)$, i.e., $\tilde{P} \in P^{*}(\hat{x})$.

Thus any sequence $P_{M}^{* k}$ in $P_{M}^{*}\left(\hat{x}^{k}\right)$ has a subsequence $P_{M_{\ell}}^{* k_{j}}$ that converges to a $\tilde{P} \in P^{*}(\hat{x})$. So $\operatorname{dist}\left(P_{M_{\ell}}^{*}\left(\hat{x}^{k_{j}}\right), P^{*}(\hat{x})\right) \rightarrow 0$ as $M_{\ell} \rightarrow \infty$ and $k_{j} \rightarrow \infty$. It follows that $\operatorname{dist}\left(P_{M}^{*}\left(\hat{x}^{k}\right), P^{*}(\hat{x})\right) \rightarrow 0$ as $M \rightarrow \infty$ if $\hat{x}^{k} \rightarrow \hat{x}$.

In order to address computational issues, we use the family of approximate minimization problems defined above. If $Q_{d}, \mathcal{P}^{M}(Q)$, and $\mathcal{P}_{d}(Q)$ are defined as in Theorem 6.2, we know from Theorem 6.1 we can approximate any $P \in \mathcal{P}(Q)$ by $P_{d} \in \mathcal{P}_{d}(Q)$. Furthermore, from the results established above we also know we can approximate 
any $P \in \mathcal{P}(Q)$ by distributions $P_{M} \in \mathcal{P}^{M}(Q)$. By choosing $M$ sufficiently large we obtain

$$
\int_{Q} x\left(t_{i} ; q\right) d P(q) \approx \int_{Q} x\left(t_{i} ; q\right) \sum_{j=1}^{M} \delta_{q_{j}^{M}}(q) d P(q)=\sum_{j=1}^{M} x\left(t_{i} ; q_{j}^{M}\right) p_{j} .
$$

Thus we can approximate $J(q)$ by

$$
J^{M}(\bar{p}) \equiv \sum_{i=1}^{n}\left|\hat{x}_{i}-\sum_{j=1}^{M} x\left(t_{i} ; q_{j}^{M}\right) p_{j}\right|^{2}
$$

where $\bar{p}=\left(p_{1}, p_{2}, \ldots, p_{M}\right)$ and $\sum_{j=1}^{M} p_{j}=1, p_{j} \geq 0, p_{j} \in \mathcal{R}, 1 \leq j \leq M$.

If we define the $n M \times 1$ column vectors, $M \times n$ matrix and $n$ vector, respectively, by

$$
\begin{aligned}
X_{i}^{M} & =\left(x\left(t_{i} ; q_{1}^{M}\right), x\left(t_{i} ; q_{2}^{M}\right), \ldots, x\left(t_{i} ; q_{M}^{M}\right)\right)^{T}, X^{M}=\left[X_{1}^{M}, \ldots, X_{n}^{M}\right] \\
\hat{X} & =\left(\hat{x}_{1}, \ldots, \hat{x}_{n}\right)
\end{aligned}
$$

then we can write

$$
J^{M}(\bar{p})=\sum_{i=1}^{n}\left|\hat{x}_{i}-\bar{p} \cdot X_{i}^{M}\right|^{2}=\left\|\hat{X}-\bar{p} \cdot X^{M}\right\|_{2}^{2} .
$$

The approximate minimization problem thus reduces to a constrained optimization problem for a quadratic cost functional. Such problems are amenable to a number of standard algorithms.

Thus we see that any solution $\bar{p}$ must necessarily satisfy the vector equation $\bar{p}$. $X^{M}=\hat{X}$ and that if $X^{M}$ is nonsingular, $\bar{p}$ is uniquely determined and depends continuously on the data $\hat{X}$.

\subsection{Estimating the Thermal Conductivity Param- eters as Random Variables}

While the formulation in the previous section allows us to estimate the distributions

for both of the thermal conductivity parameters, $k_{p}$ and $k_{s}$, at the same time, we 
will estimate them individually. We first assume the parameter $k_{p}$ is a realization for a normally distributed random variable with mean $\mu_{p}$ and variance $\sigma_{p}^{2}$ and attempt to estimate its distribution. For now, we will hold all other parameters constant. In order to ensure all values in the distribution for $k_{p}$ are positive we will use a truncated Gaussian distribution and require $\mu_{p}-3 \sigma_{p}>0$. Thus the probability density function for the random variable is given by

$$
f(x)=\frac{1}{0.9974 \sigma_{p} \sqrt{2 \pi}} e^{-\left(x-\mu_{p}\right)^{2} / 2 \sigma_{p}^{2}}
$$

for $x \in\left[\mu_{p}-3 \sigma_{p}, \mu_{p}+3 \sigma_{p}\right]$. If we define $q_{p}=\left(\mu_{p}, \sigma_{p}\right)$, we can define

$$
\mathcal{E}\left[T\left(t_{i} ; q_{p}\right) \mid P_{p}\right]=\int_{\mu_{p}-3 \sigma_{p}}^{\mu_{p}+3 \sigma_{p}} T\left(t_{i} ; x\right) \frac{1}{0.9974 \sigma_{p} \sqrt{2 \pi}} e^{-\left(x-\mu_{p}\right)^{2} / 2 \sigma_{p}^{2}} d x
$$

where $P_{p}$ is the probability distribution function arising from (6.19) with mean $\mu_{p}$ and variance $\sigma_{p}^{2}$. Thus the "best fit" parameter $q_{p}^{*}$ is the solution to the least squares problem

$$
\min _{q_{p} \in Q} J\left(P_{p}, \hat{T}\right) \equiv \min _{q_{p} \in Q} J\left(P_{p}\right) \equiv \min _{q_{p} \in Q} \sum_{i=1}^{n}\left|\mathcal{E}\left[T\left(t_{i} ; q_{p}\right) \mid P_{p}\right]-\hat{T}_{i}\right|^{2}
$$

where $Q=\mathbb{R}^{+} \times \mathbb{R}^{+}$with the additional restriction $\mu_{p}-3 \sigma_{p}>0$.

For proof of concept, we will use the same generated data described in Section 6.1. While we realize the data was not generated with $k_{p}$ normally distributed, we would consider a successful parameter estimation to have the mean of the distribution near the true value for $k_{p}$ and small standard deviation. In Table 6.3 we see values for two minimizations of (6.20). As in the constant parameter estimation problem, we use Matlab's constrained minimization routine fmincon. The initial guess is $q_{p_{0}}=$ $\left(\mu_{p_{0}}, \sigma_{p_{0}}\right)$ and the best parameter fit found by Matlab is $q_{p}^{*}=\left(\mu_{p}^{*}, \sigma_{p}^{*}\right)$. For both of these minimizations, $n=5$ in (6.20).

In both cases, the mean of the "best fit" distribution does not closely resemble the actual parameter value, $k_{p}=217$ and the standard deviation is not small. In both cases, the optimal parameters are not far from the initial guesses, as we saw in the 


\begin{tabular}{|c|c|c|c|c|c|}
\hline$\mu_{p_{0}}$ & $\sigma_{p_{0}}$ & $J\left(q_{p_{0}}\right)$ & $\mu_{p}^{*}$ & $\sigma_{p}^{*}$ & $J\left(q_{p}^{*}\right)$ \\
\hline \hline 1000 & 100 & 0.0197193 & 1242.66 & 297.228 & 0.0153633 \\
\hline 250 & 50 & 0.0391095 & 250.001 & 50.0005 & 0.0391080 \\
\hline
\end{tabular}

Table 6.3: Estimating distribution of $k_{p}$

constant parameter estimation for $k_{p}$. While we are using a relatively small number for $n$ and a constant distribution, we would still expect better results.

In contrast, suppose we instead view $k_{s}$ as a realization for a normally distributed random variable and estimate $\mu_{s}$ and $\sigma_{s}$ for the distribution. As before we use a truncated Gaussian to ensure all possible values are positive, with probability density function

$$
f(x)=\frac{1}{0.9974 \sigma_{s} \sqrt{2 \pi}} e^{-\left(x-\mu_{s}\right)^{2} / 2 \sigma_{s}^{2}}
$$

for $x \in\left[\mu_{s}-3 \sigma_{s}, \mu_{s}+3 \sigma_{s}\right], \mu_{s}-3 \sigma_{s}>0$. If we define $q_{s}=\left(\mu_{s}, \sigma_{s}\right)$, we can define

$$
\mathcal{E}\left[T\left(t_{i} ; q_{s}\right) \mid P_{s}\right]=\int_{\mu_{s}-3 \sigma_{s}}^{\mu_{s}+3 \sigma_{s}} T\left(t_{i} ; x\right) \frac{1}{0.9974 \sigma_{s} \sqrt{2 \pi}} e^{-\left(x-\mu_{s}\right)^{2} / 2 \sigma_{s}^{2}} d x
$$

where $P_{s}$ is the probability distribution function arising from (6.21) with mean $\mu_{s}$ and variance $\sigma_{s}^{2}$. Thus the "best fit" parameter $q_{s}^{*}$ is the solution to the least squares problem

$$
\min _{q_{s} \in Q} J\left(P_{s}, \hat{T}\right) \equiv \min _{q_{s} \in Q} J\left(P_{s}\right) \equiv \min _{q_{s} \in Q} \sum_{i=1}^{n}\left|\mathcal{E}\left[T\left(t_{i} ; q_{s}\right) \mid P_{s}\right]-\hat{T}_{i}\right|^{2}
$$

where $Q=\mathbb{R}^{+} \times \mathbb{R}^{+}$with the additional restriction $\mu_{p}-3 \sigma_{p}>0$.

In Table 6.4 we see values for one minimization of (6.22). We use Matlab and the generated data as before. The initial guess is $q_{s_{0}}=\left(\mu_{s_{0}}, \sigma_{s_{0}}\right)$ and the best parameter fit found by Matlab is $q_{s}^{*}=\left(\mu_{s}^{*}, \sigma_{s}^{*}\right)$. For both of these minimizations, $n=5$ in (6.22).

In this example, note the mean $\mu_{s}^{*}$ of the distribution is very close to the actual value of $k_{s}=0.12 \mathrm{~W} / \mathrm{mK}$ and the standard deviation is small. This indicates that despite the fact we are using a small number for $n$, the parameter estimation algorithm performs well when estimating $k_{s}$ (or its distribution). 
Chapter 6. Parameter Estimation for Two Dimensional Heat Transfer Problem 90

\begin{tabular}{|c|c|c|c|c|c|}
\hline$\mu_{s_{0}}$ & $\sigma_{s_{0}}$ & $J\left(q_{s_{0}}\right)$ & $\mu_{s}^{*}$ & $\sigma_{s}^{*}$ & $J\left(q_{S}^{*}\right)$ \\
\hline \hline 1 & 0.25 & 19.294 & 0.12036 & $2.5087 \mathrm{e}-05$ & $1.4140 \mathrm{e}-02$ \\
\hline
\end{tabular}

Table 6.4: Estimating distribution of $k_{s}$ 


\section{Chapter 7}

\section{Sensitivity Equations}

In the previous chapter we saw that small changes in the parameter for the thermal conductivity of the particles, $k_{p}$, seem to have little impact on the solution $u(t, z)$. In contrast, small changes in the thermal conductivity of the silicone, $k_{s}$, appear to significantly change the solution, $u(t, z)$. Sensitivity equations allow us to describe how changes in the parameters affect changes in the solutions. Thus, we turn to sensitivity equations to see if $k_{p}$ and $k_{s}$ are, in fact, influencing the solutions as we suspect. See $[25,26,27,28]$ for more information on sensitivity equation methods.

\subsection{Formal Derivation using Fréchet Derivatives}

In order to derive the sensitivity equations, we must formally differentiate our system of equations, including the boundary conditions, and then interchange the order of differentiation. Before explicitly following this procedure, we first rigorously justify the derivation. This derivation relies on the Implicit Function Theorem and a corollary to the Implicit Function Theorem, both of which we state here.

Theorem 7.1 (Implicit Function Theorem) [9, Theorem 3.1.10, p.115] Let X, $Y$, and $Z$ be Banach spaces. Suppose $f(x, y)$ is a continuous mapping of a neighborhood $U$ of $\left(x_{0}, y_{0}\right)$ in $X \times Y$ into $Z, f\left(x_{0}, y_{0}\right)=0$ and $f_{y}\left(x_{0}, y_{0}\right)$ exists, is continuous in $x$, and is a linear homeomorphism of $Y$ onto $Z$. Then there is a unique continuous 
mapping $g$ defined in a neighborhood $U_{1}$ of $x_{0}, g: U_{1} \rightarrow Y$, such that $g\left(x_{0}\right)=y_{0}$ and $f(x, g(x))=0$ for $x \in U_{1}$.

Corollary 7.2 [9, Corollary 3.1.11, p.115] If, in addition to the hypothesis of the Implicit Function Theorem $f_{x}(x, y)$ exists and is continuous for $(x, y)$ near $\left(x_{0}, y_{0}\right)$, then the function $g(x)$ is continuously differentiable for $x \in U_{1}$ and

$$
g^{\prime}(x)=-\left[f_{y}(x, g(x))\right]^{-1} f_{x}(x, g(x))
$$

A proof of Theorem 7.1 and Corollary 7.2 can be found in [9].

In Section 5.2.1 we showed there exists a unique, weak solution to

$$
\left\{\begin{array}{l}
\dot{u}+A u=F \\
u(0)=u_{0}
\end{array}\right.
$$

where $F$ is defined in (5.9) and $A$ is given by (5.8). Without loss of generality, we assume $u(0)=0$. (If not use a simple change of variables, $\hat{u}=u-u(0)$.) We are interested in examining the sensitivity of these solutions with respect to the thermal conductivity parameter $k$, or, more specifically, with respect to parameters $q$ in a parameterization $k(q)$ of the thermal conductivity. Let $Q$ be the space of all possible parameter values, $\boldsymbol{y}=L^{2}\left(0, T ; \mathcal{V}^{*}\right)$ and $\mathcal{U}=L^{2}(0, T ; \mathcal{V}) \cap H^{1}\left(0, T ; \mathcal{V}^{*}\right)$. The norm on $\mathcal{U}$ is given by

$$
\|v\|_{u}^{2}=\|v\|_{L^{2}(0, T ; \mathcal{V})}^{2}+\|v\|_{H^{1}\left(0, T ; \mathcal{V}^{*}\right)}^{2}
$$

for $v \in \mathcal{U}($ see $[19$, p. 102]).

Note $F$ as defined in (5.9) does not depend on $q$. The thermal conductivity does depend on $q, k=k(q): Q \rightarrow \mathbb{R}$ and so by (5.8) we have $A=A(q): Q \rightarrow \mathscr{L}\left(\mathcal{V}, \mathcal{V}^{*}\right)$, with

$$
\langle A(q) \phi, \psi\rangle_{\mathcal{V}^{*}, \mathcal{V}}=\left\langle\frac{1}{g} k(q) \nabla \phi, \nabla \psi\right\rangle_{\mathcal{H}}+h \int_{\gamma_{2}}\left(\operatorname{Tr}_{2} \phi\right)(z)\left(\operatorname{Tr}_{2} \psi\right)(z) d S_{2}
$$

for $\phi, \psi \in \mathcal{V}$. Thus for each $q \in Q$ there is an associated weak solution $u(\cdot ; q) \in \mathcal{U}$. 
Define $M: Q \rightarrow \mathscr{L}(\mathcal{U}, \mathrm{y})$ by

$$
[M(q)] v=\dot{v}+A(q) v
$$

Since (7.2) possesses a unique solution for each $F \in L^{2}\left(0, T ; \mathcal{V}^{*}\right)$, we see that $M(q)$ is invertible for each $q \in Q$. Note also $M(q)$ is linear in $v$, i.e., $[M(q)]\left(v_{1}+v_{2}\right)=$ $[M(q)] v_{1}+[M(q)] v_{2}$. Define $N: Q \rightarrow \mathrm{y}$ by

$$
N(q)=F
$$

Finally, define $G: \mathcal{U} \times Q \rightarrow \mathcal{y}$ by

$$
G(u, q)=([M(q)] u)-[N(q)]
$$

and associate $u(\cdot ; q)$ with the pair $(u(\cdot ; q), q)$ satisfying $G(u(\cdot ; q), q)=0$ in the $y=L^{2}\left(0, T ; \mathcal{V}^{*}\right)$ sense. Note $G$ induces a natural mapping from $Q$ to $\mathcal{U}$ given by $q \mapsto u(\cdot ; q)$. Also, $u \mapsto G(u(\cdot, q), q)$ is an affine map from $\mathcal{U}$ to $\boldsymbol{y}$.

Fix $q_{0} \in Q$ and let $u_{0} \equiv u_{0}\left(\cdot ; q_{0}\right) \in \mathcal{U}$. In order to characterize the sensitivity at $q_{0}$ we need the operator $D_{q} u\left(q_{0}\right) \in \mathscr{L}(Q, \mathcal{U})$. We assume $\left(u_{0}, q_{0}\right) \equiv\left(u_{0}\left(\cdot ; q_{0}\right), q_{0}\right)$ satisfies $G\left(u_{0}, q_{0}\right)=0$ in the $y=L^{2}\left(0, T ; \mathcal{V}^{*}\right)$ sense.

Lemma 7.3 The partial Fréchet derivative $\partial_{u} G\left(u_{0}, q_{0}\right): \mathcal{U} \rightarrow \mathrm{y}$ exists and is given by $\partial_{u} G\left(u_{0}, q_{0}\right)=M\left(q_{0}\right) \in \mathscr{L}(\mathcal{U}, y)$.

Proof: For $h \neq 0 \in \mathcal{U}$,

$$
\begin{aligned}
\| G\left(u_{0}+h, q_{0}\right) & -G\left(u_{0}, q_{0}\right)-M\left(q_{0}\right) h \|_{y} \\
& =\left\|\left[M\left(q_{0}\right)\right]\left(u_{0}+h\right)-\left[N\left(q_{0}\right)\right]-\left(\left[M\left(q_{0}\right)\right] u_{0}-\left[N\left(q_{0}\right)\right]\right)-\left[M\left(q_{0}\right)\right] h\right\|_{y} \\
& =\left\|\left[M\left(q_{0}\right)\right] h-\left[M\left(q_{0}\right)\right] h\right\|_{y} \\
& =0 .
\end{aligned}
$$

We assume the function $k: Q \rightarrow \mathbb{R}$ is Fréchet differentiable at $q_{0}$ and so we define $\mathscr{M}: Q \rightarrow \mathscr{L}(Q, \mathscr{L}(\mathcal{U}, y))$ by

$$
\left[\mathscr{M}\left(q_{0}\right) u\right]=\mathscr{A}\left(q_{0}\right) u
$$


where $\mathscr{A}: Q \rightarrow \mathscr{L}\left(Q, \mathscr{L}\left(\mathcal{V}, \mathcal{V}^{*}\right)\right)$ is given by

$$
\left\langle\mathscr{A}\left(q_{0}\right) \phi, \psi\right\rangle_{\mathcal{V}^{*}, \mathcal{V}}=\left\langle\frac{1}{g} D_{q} k\left(q_{0}\right) \nabla \phi, \nabla \psi\right\rangle_{\mathcal{H}}
$$

for $\phi, \psi \in \mathcal{V}$. Note that $k: Q \rightarrow \mathbb{R}$ and so $D_{q} k\left(q_{0}\right) \in \mathscr{L}(Q, \mathbb{R})$.

Let $\mathscr{X}=\mathscr{L}(\mathcal{U}, \mathcal{y})$. Since $\mathcal{y}$ and $\mathcal{U}$ are Banach spaces, $\mathscr{X}$ is a Banach space as well. Thus we can define a norm on $\mathscr{X}$ by

$$
\|T\|_{\mathscr{X}}=\sup _{\|v\|_{u=1}}\|T v\|_{y}
$$

for $T \in \mathscr{X}$. Recall for $\zeta \in \mathcal{V}^{*}$

$$
\|\zeta\|_{\mathcal{V}^{*}}=\sup _{\|\psi\|_{\mathcal{v}=1}}\langle\zeta, \psi\rangle_{\mathcal{V}^{*}, \mathcal{V}}
$$

and for $\phi \in \mathcal{V}$

$$
\|\phi\|_{\mathcal{V}}^{2}=\|\nabla \phi\|_{\mathcal{H}}^{2}+\|\phi\|_{L^{2}}^{2}
$$

Recall from (5.2) that $g$ is positive and finite and note that for any $\phi \in \mathcal{V}$,

$$
\begin{aligned}
\|\nabla \phi\|_{L^{2}(\Omega)}^{2} & =\langle\nabla \phi, \nabla \phi\rangle_{L^{2}(\Omega)} \\
& =\left\langle\frac{1}{g} \cdot g \nabla \phi, \nabla \phi\right\rangle_{L^{2}(\Omega)} \\
& =\left\langle\frac{1}{g} \nabla \phi, \nabla \phi\right\rangle_{\mathcal{H}} \\
& \leq R_{L}^{-1}\|\nabla \phi\|_{\mathcal{H}}^{2} \\
& \leq R_{L}^{-1}\|\phi\|_{\mathcal{V}}^{2}
\end{aligned}
$$

and so $\|\nabla \phi\|_{L^{2}(\Omega)} \leq \sqrt{R_{L}^{-1}}\|\phi\|_{\mathcal{v}}$

Lemma 7.4 $M$ is Fréchet differentiable at $q_{0}$, with $\left[\mathscr{M}\left(q_{0}\right) u\right] h=\left[D_{q} M\left(q_{0}\right) h\right] u$. Furthermore, $\mathscr{M}\left(q_{0}\right)$ is a bounded linear operator. 
Proof: For $h \neq 0 \in Q$ we want to show

$$
\lim _{h \rightarrow 0} \frac{1}{\|h\|_{Q}}\left\|M\left(q_{0}+h\right)-M\left(q_{0}\right)-\left[\mathscr{M}\left(q_{0}\right)\right] h\right\|_{\mathscr{X}}=0 .
$$

By definition of the norm in $\mathscr{X}$,

$$
\left\|M\left(q_{0}+h\right)-M\left(q_{0}\right)-\left[\mathscr{M}\left(q_{0}\right)\right] h\right\|_{\mathscr{X}}=\sup _{\|u\|_{u=1}}\left\|\left[M\left(q_{0}+h\right) u-M\left(q_{0}\right) u-\left[\mathscr{M}\left(q_{0}\right) u\right] h\right]\right\| y .
$$

For any $u \in \mathcal{U}$ with $\|u\|_{u}=1$,

$$
\begin{aligned}
\|[M & \left.\left(q_{0}+h\right) u-M\left(q_{0}\right) u-\left[\mathscr{M}\left(q_{0}\right) u\right] h\right] \|_{y}^{2} \\
& =\int_{0}^{T}\left\|\left(\left[M\left(q_{0}+h\right)\right] u\right)(t)-\left(\left[M\left(q_{0}\right)\right] u\right)(t)-\left(\left[\mathscr{M}\left(q_{0}\right) u\right] h\right)(t)\right\|_{\mathcal{V}^{*}}^{2} d t \\
& =\int_{0}^{T}\left\|\dot{u}(t)+A\left(q_{0}+h\right) u(t)-\left[\dot{u}(t)+A\left(q_{0}\right) u(t)\right]-\mathscr{A}\left(q_{0}\right) u(t) h\right\|_{\mathcal{V}^{*}}^{2} d t \\
& =\int_{0}^{T} \sup _{\|\psi\|_{\mathcal{V}}=1}\left\langle A\left(q_{0}+h\right) u(t)-A\left(q_{0}\right) u(t)-\mathscr{A}\left(q_{0}\right) u(t) h, \psi\right\rangle_{\mathcal{V}^{*}, \mathcal{V}}^{2} d t \\
& =\int_{0}^{T} \sup _{\|\psi\|_{\mathcal{V}}=1}\left\langle\frac{1}{g} k\left(q_{0}+h\right) \nabla u(t)-\frac{1}{g} k\left(q_{0}\right) \nabla u(t)-\frac{1}{g} D_{q} k\left(q_{0}\right) h \nabla u(t), \nabla \psi\right\rangle_{\mathcal{H}}^{2} d t \\
& \leq \int_{0}^{T} \sup _{\|\psi\|_{\mathcal{V}}=1}\left\|k\left(q_{0}+h\right)-k\left(q_{0}\right)-D_{q} k\left(q_{0}\right) h\right\|_{\infty}^{2}\langle\nabla u(t), \nabla \psi\rangle_{L^{2}(\Omega)}^{2} d t \\
& \leq \sup _{\|\psi\|_{\mathcal{V}}=1}\left\|k\left(q_{0}+h\right)-k\left(q_{0}\right)-D_{q} k\left(q_{0}\right) h\right\|_{\infty}^{2}\|\nabla \psi\|_{L^{2}(\Omega)}^{2} \int_{0}^{T}\|\nabla u(t)\|_{L^{2}(\Omega)}^{2} d t \\
\leq & \sup _{\|\psi\|}\left\|k\left(q_{0}+h\right)-k\left(q_{0}\right)-D_{q} k\left(q_{0}\right) h\right\|_{\infty}^{2} R_{L}^{-1}\|\psi\|_{\mathcal{V}}^{2} \int_{0}^{T} R_{L}^{-1}\|u(t)\|_{\mathcal{V}}^{2} d t \\
& =\left\|k\left(q_{0}+h\right)-k\left(q_{0}\right)-D_{q} k\left(q_{0}\right) h\right\|_{\infty}^{2} R_{L}^{-2}\|u\|_{L^{2}(0, T ; \mathcal{V})}^{2} \\
\leq & \left\|k\left(q_{0}+h\right)-k\left(q_{0}\right)-D_{q} k\left(q_{0}\right) h\right\|_{\infty}^{2} R_{L}^{-2}\|u\|_{\mathcal{u}}^{2}
\end{aligned}
$$

Thus

$$
\left\|M\left(q_{0}+h\right)-M\left(q_{0}\right)-\left[\mathscr{M}\left(q_{0}\right)\right] h\right\|_{\mathscr{X}} \leq\left\|k\left(q_{0}+h\right)-k\left(q_{0}\right)-D_{q} k\left(q_{0}\right) h\right\|_{\infty} R_{L}^{-1} .
$$


We know

$$
\lim _{h \rightarrow 0} \frac{1}{\|h\|_{Q}}\left\|k\left(q_{0}+h\right)-k\left(q_{0}\right)-D_{q} k\left(q_{0}\right) h\right\|_{\infty}=0
$$

since we assume $k$ is Fréchet differentiable. Thus

$$
\lim _{h \rightarrow 0} \frac{1}{\|h\|_{Q}}\left\|M\left(q_{0}+h\right)-M\left(q_{0}\right)-\left[\mathscr{M}\left(q_{0}\right)\right] h\right\|_{\mathscr{X}}=0
$$

and so $M$ is Fréchet differentiable with $D_{q} M=\mathscr{M}$ and $\mathscr{M}\left(q_{0}\right) \in \mathscr{L}(Q, \mathscr{L}(\mathcal{U}, y))=$ $\mathscr{L}(Q, \mathscr{X})$.

Lemma 7.5 If $D_{q} N\left(q_{0}\right): Q \rightarrow \mathrm{y}$ and $D_{q} M\left(q_{0}\right): Q \rightarrow \mathscr{L}(\mathcal{U}, \mathrm{y})$ exist in the Fréchet sense, then the partial derivative of $G$ with respect to $Q$ at $\left(u_{0}, q_{0}\right)$ exists and $\left[\partial_{q} G\left(u_{0}, q_{0}\right)\right] \in \mathscr{L}(Q, y)$ is given by

$$
\left[\partial_{q} G\left(u_{0}, q_{0}\right)\right]=\mathscr{M}\left(q_{0}\right) u_{0}-D_{q} N\left(q_{0}\right)
$$

Proof: By Lemma 7.4, $D_{q} M\left(q_{0}\right)=\mathscr{M}\left(q_{0}\right) \in \mathscr{L}(Q, \mathscr{L}(\mathcal{U}, y))$. Since $N\left(q_{0}\right)$ has no dependence on $q, D_{q} N\left(q_{0}\right)$ is the zero operator and so $D_{q} N\left(q_{0}\right) \in \mathscr{L}(Q, y)$. Thus $\mathscr{M}\left(q_{0}\right) u_{0}-D_{q} N\left(q_{0}\right) \in \mathscr{L}(Q, y)$.

For $h \neq 0 \in Q$,

$$
\begin{aligned}
& \| G\left(u_{0}, q_{0}+h\right)- G\left(u_{0}, q_{0}\right)-\left[\mathscr{M}\left(q_{0}\right) u_{0}-D_{q} N\left(q_{0}\right)\right] h \|_{y} \\
&=\left.\|\left[M\left(q_{0}+h\right)\right] u_{0}-N\left(q_{0}+h\right)-\left(\left[M\left(q_{0}\right)\right] u_{0}-N\left(q_{0}\right)\right]\right) \\
&-\left(\left[D_{q} M\left(q_{0}\right) h\right] u_{0}-D_{q} N\left(q_{0}\right) h \|_{y}\right. \\
& \leq\left\|M\left(q_{0}+h\right)-M\left(q_{0}\right)-D_{q} M\left(q_{0}\right) h\right\| \mathscr{X}\left\|u_{0}\right\|_{u} \\
&+\left\|N\left(q_{0}+h\right)-N\left(q_{0}\right)-D_{q} N\left(q_{0}\right) h\right\|_{y}
\end{aligned}
$$

Since $D_{q} M\left(q_{0}\right)$ exists in the Fréchet sense,

$$
\lim _{h \rightarrow 0} \frac{1}{\|h\|_{Q}}\left\|M\left(q_{0}+h\right)-M\left(q_{0}\right)-D_{q} M\left(q_{0}\right) h\right\|_{\mathscr{X}}=0 .
$$

Moreover, since $N$ has no dependence on $q$,

$$
N\left(q_{0}+h\right)-N\left(q_{0}\right)-D_{q} N\left(q_{0}\right) h \equiv 0 .
$$


Thus

$$
\lim _{h \rightarrow 0} \frac{1}{\|h\|_{Q}}\left\|G\left(u_{0}, q_{0}+h\right)-G\left(u_{0}, q_{0}\right)-\left[\mathscr{M}\left(q_{0}\right) u_{0}-D_{q} N\left(q_{0}\right)\right] h\right\|_{y}=0
$$

and so $\left[\partial_{q} G\left(u_{0}, q_{0}\right)\right]=\mathscr{M}\left(q_{0}\right) u_{0}-D_{q} N\left(q_{0}\right)$.

Theorem 7.6 Let $Q_{0}$ be a subset of the interior of $Q$ and let $\mathcal{U}_{0}$ be a subset of the interior of $\mathcal{U}$. Fix $q_{0} \in Q_{0} \subset Q$. Suppose there exists a unique $u_{0}\left(\cdot ; q_{0}\right) \in \mathcal{U}_{0} \subset \mathcal{U}$ such that $G\left(u_{0}\left(\cdot ; q_{0}\right), q_{0}\right)=0$. If $\left[M\left(q_{0}\right)\right]^{-1}$ exists in $\mathscr{L}(\mathcal{y}, \mathcal{U})$ and if $D_{q} M\left(q_{0}\right)$ and $D_{q} N\left(q_{0}\right)$ exist in the Fréchet sense in $\mathscr{L}(Q, \mathscr{X})$ and $\mathscr{L}(Q, y)$ respectively, then the sensitivity operator, $s \equiv D_{q} u\left(\cdot ; q_{0}\right) \in \mathscr{L}(Q, \mathcal{U})$, exists and satisfies

$$
M\left(q_{0}\right) s=-\mathscr{M}\left(q_{0}\right) u_{0}+D_{q} N\left(q_{0}\right)
$$

Proof: In the Implicit Function Theorem 7.1, we set $X=Q, Y=\mathcal{U}$, and $Z=y$. The function $f(x, y)$ in Theorem 7.1 is our $G(u, q)$, and by assumption $G\left(u_{0}, q_{0}\right)=0$. By Lemma 7.3, we know $\partial_{u} G\left(u_{0}, q_{0}\right) \in \mathscr{L}(\mathcal{U}, y)$. Since $M(q)$ is invertible for each $q \in Q$, $\left[M\left(q_{0}\right)\right]^{-1} \in \mathscr{L}(\mathcal{y}, \mathcal{U})$. Thus $u(\cdot, q)$ satisfies $G(u(\cdot, q), q)=0$ for $q \in Q_{0}$.

By Lemma 7.5, we know $\partial_{q} G\left(u_{0}, q_{0}\right) \in \mathscr{L}(Q, y)$. Furthermore, by Lemma 7.4 we know $D_{q} M\left(q_{0}\right)=\mathscr{M}\left(q_{0}\right)$ and so by Corollary $7.2, M\left(q_{0}\right) s=-\mathscr{M}\left(q_{0}\right) u_{0}+D_{q} N\left(q_{0}\right)$. In fact, since $D_{q} N\left(q_{0}\right)$ is the zero operator, $M\left(q_{0}\right) s=-\mathscr{M}\left(q_{0}\right) u_{0}$. 


\subsection{Sensitivity to the Particle Thermal Conduc- tivity}

\subsubsection{Derivation of Sensitivity Equations}

We return to our two dimensional model from Section 3.2:

$$
\begin{cases}g(z) \dot{u}(t, z)=\nabla \cdot(k(z) \nabla u(t, z)) & \\ \left.k(z) \frac{\partial u}{\partial n}(t, z)\right|_{\gamma_{4}}=S_{0}(t) & \text { (source) } \\ \left.k(z) \frac{\partial u}{\partial n}(t, z)\right|_{\gamma_{2}}=\left.h\left(T_{\infty}-u(t, z)\right)\right|_{\gamma_{2}} & (\text { sink }) \\ \left.k(z) \frac{\partial u}{\partial n}(t, z)\right|_{\gamma_{1}}=0 & \\ \left.k(z) \frac{\partial u}{\partial n}(t, z)\right|_{\gamma_{3}}=0 & \\ u(0, z)=\Upsilon(z) & \end{cases}
$$

for $z \in \Omega$ and $t \in[0, T]$ and where $\partial \Omega=\gamma_{1} \cup \gamma_{2} \cup \gamma_{3} \cup \gamma_{4}$ as depicted in Figure 3.4.

In Section 5.1.2 we showed the system (7.3) has a weak solution $u(t, z)$. If we assume the thermal conductivity is dependent on the parameter $q$, then $q \mapsto k(z ; q)$ is given by

$$
k(z ; q)= \begin{cases}\bar{q}_{s} & z \in \Omega_{s} \\ q_{p} & z \in \Omega_{p} .\end{cases}
$$

We want to consider the thermal properties of the composite silicone as we hold the constant $\bar{q}_{s}$ fixed and let the constant $q_{p}$ vary over a range of admissible material values. Thus any weak solution of (7.3) will have the form $u(t, z ; q)$. By studying the sensitivity of the solution $u$ to changes in $q_{p}$, we can study the sensitivity of the system to the thermal conductivity of the particles.

In order to derive the sensitivity equations, we will formally differentiate (7.3) with respect to $q_{p}$, interchange the order of differentiation and define the sensitivity. Our analysis in Section 7.1 guarantees the existence of these derivatives and the resulting sensitivity equation can be rigorously interpreted in terms of an associated weak or variational system. 
First, formally differentiating $g(z) \dot{u}(t, z)=\nabla \cdot(k(z) \nabla u(t, z))$ with respect to $q_{p}$ yields

$$
g(z) \frac{\partial}{\partial q_{p}}(\dot{u}(t, z ; q))=\frac{\partial}{\partial q_{p}}(\nabla \cdot(k(z ; q) \nabla u(t, z ; q))) .
$$

Switching the order of differentiation we see

$$
g(z) \frac{\partial}{\partial t}\left(\frac{\partial u}{\partial q_{p}}(t, z ; q)\right)=\nabla \cdot\left(\frac{\partial k}{\partial q_{p}}(z ; q) \nabla u(t, z ; q)\right)+\nabla \cdot\left(k(z ; q) \nabla \frac{\partial u}{\partial q_{p}}(t, z ; q)\right) .
$$

If we then define the sensitivity to $q_{p}$ as $s(t, z ; q)=\frac{\partial u}{\partial q_{p}}(t, z ; q)$ and substitute into the previous equation we have

$$
g(z) \dot{s}(t, z ; q)=\nabla \cdot\left(\frac{\partial k}{\partial q_{p}}(z ; q) \nabla u(t, z ; q)\right)+\nabla \cdot(k(z ; q) \nabla s(t, z ; q)) .
$$

It is also necessary to differentiate the boundary conditions with respect to $q_{p}$. To do this, note

$$
\begin{aligned}
\frac{\partial}{\partial q_{p}}\left(k(z ; q) \frac{\partial u}{\partial n}(t, z ; q)\right) & =\frac{\partial k}{\partial q_{p}}(z ; q) \frac{\partial u}{\partial n}(t, z ; q)+k(z ; q) \frac{\partial}{\partial n}\left(\frac{\partial u}{\partial q_{p}}(t, z ; q)\right) \\
& =k(z ; q) \frac{\partial s}{\partial n}(t, z ; q)+\frac{\partial k}{\partial q_{p}}(z ; q) \frac{\partial u}{\partial n}(t, z ; q) .
\end{aligned}
$$

We will assume the source flux, $S_{0}(t)$ and the initial condition, $\Upsilon(z)$ are independent of $q_{p}$.

It is important to note that

$$
\frac{\partial k}{\partial q_{p}}(z ; q)= \begin{cases}0 & z \in \Omega_{s} \\ 1 & z \in \Omega_{p}\end{cases}
$$

and so $\frac{\partial k}{\partial q_{p}}(z ; q) \in L^{\infty}(\Omega)$. Furthermore, $\frac{\partial k}{\partial q_{s}}(z ; q)=0$ since we are holding $q_{s}=\bar{q}_{s}$ fixed. Since $q=\left(q_{p}, q_{s}\right), D_{q} k(q)=\left(\frac{\partial k}{\partial q_{p}}, \frac{\partial k}{\partial q_{s}}\right)=\left(\frac{\partial k}{\partial q_{p}}, 0\right)$. Also, since $u \in L^{2}(0, T ; \mathcal{V})$, we know $\nabla u \in L^{2}\left(0, T ; L^{2}(\Omega)^{2}\right)$. Then if we define

$$
f(t, z ; q)=\frac{\partial k}{\partial q_{p}}(z ; q) \nabla u(t, z ; q)
$$

we have $f(\cdot, \cdot ; q) \in L^{2}\left(0, T ; L^{2}(\Omega)^{2}\right)$. 
Thus we formally have the following system for our sensitivity equation:

$$
\left\{\begin{array}{l}
g(z) \dot{s}(t, z ; q)=\nabla \cdot(k(z ; q) \nabla s(t, z ; q))+\nabla \cdot f(t, z ; q) \\
\left.k(z ; q) \frac{\partial s}{\partial n}(t, z ; q)\right|_{\gamma_{4}}=-\left.\frac{\partial k}{\partial q_{p}}(z ; q) \frac{\partial u}{\partial n}(t, z ; q)\right|_{\gamma_{4}} \\
\left.k(z ; q) \frac{\partial s}{\partial n}(t, z ; q)\right|_{\gamma_{2}}=-\left.\left(\frac{\partial k}{\partial q_{p}}(z ; q) \frac{\partial u}{\partial n}(t, z ; q)+h s(t, z ; q)\right)\right|_{\gamma_{2}} \\
\left.k(z ; q) \frac{\partial s}{\partial n}(t, z ; q)\right|_{\gamma_{1}}=-\left.\frac{\partial k}{\partial q_{p}}(z ; q) \frac{\partial u}{\partial n}(t, z ; q)\right|_{\gamma_{1}} \\
\left.k(z ; q) \frac{\partial s}{\partial n}(t, z ; q)\right|_{\gamma_{3}}=-\left.\frac{\partial k}{\partial q_{p}}(z ; q) \frac{\partial u}{\partial n}(t, z ; q)\right|_{\gamma_{3}} \\
s(0, z ; q)=0
\end{array}\right.
$$

Note the solution $u(t, z ; q)$ to $(7.3)$ acts as part of the forcing term $f$ on the solution to $(7.6)$.

\subsubsection{Weak Solution to Sensitivity Equation}

We refer to the spaces $H, V, \mathcal{H}$, and $\mathcal{V}$ defined in Section 5.1.1. Define a sesquilinear form $\beta: \mathcal{V} \times \mathcal{V} \rightarrow \mathbb{R}$ by

$$
\beta(\phi, \psi)=\left\langle\frac{1}{g} k \nabla \phi, \nabla \psi\right\rangle_{\mathcal{H}}+h \int_{\gamma_{2}}\left(\operatorname{Tr}_{2} \phi\right)(z)\left(\operatorname{Tr}_{2} \psi\right)(z) d S_{2}
$$

and note $\beta$ defines an operator $\mathcal{A} \in \mathscr{L}\left(\mathcal{V}, \mathcal{V}^{*}\right)$ where $\langle\mathcal{A} \phi, \psi\rangle_{\mathcal{V}^{*}, \mathcal{V}}=\beta(\phi, \psi)$. We observe that $\beta=\beta(q)$ is just the parameter dependent sesquilinear form $\sigma$ of (5.8) and $\mathcal{A}=\mathcal{A}(q)$ is the analog of $A$ generated by $\sigma$.

Define $\mathcal{F}:[0, T] \rightarrow \mathcal{V}^{*}$ by

$$
[\mathcal{F}(t)](\psi)=\left\langle-\frac{1}{g} f, \nabla \psi\right\rangle_{\mathcal{H}}
$$

where $f$ is given in (7.5). We shall see shortly that $\mathcal{A}$ and $\mathcal{F}$ are the operators we need to find the weak or variational form of (7.6).

Suppose s solves

$$
\dot{s}+\mathcal{A} s=\mathcal{F} \quad \text { in } \mathcal{V}^{*},
$$

i.e., for all $\psi \in \mathcal{V}$,

$$
\langle\dot{s}(t)+\mathcal{A} s(t)-\mathcal{F}(t), \psi\rangle_{\mathcal{V}^{*}, \mathcal{V}}=0 .
$$


So, by definition

$$
\langle\dot{s}(t)+\mathcal{A} s(t), \psi\rangle_{\mathcal{V}^{*}, \mathcal{V}}=\langle\mathcal{F}(t), \psi\rangle_{\mathcal{V}^{*}, \mathcal{V}}
$$

and thus

$$
\begin{aligned}
\langle\dot{s}(t), \psi\rangle_{\mathcal{V}^{*}, \mathcal{V}}=\langle- & \left.\frac{1}{g} k \nabla s(t), \nabla \psi\right\rangle_{\mathcal{H}}-h \int_{\gamma_{2}}\left(\operatorname{Tr}_{2} s(t)\right)(z)\left(\operatorname{Tr}_{2} \psi\right)(z) d S_{2} \\
& +\left\langle-\frac{1}{g} f(t), \nabla \psi\right\rangle_{\mathcal{H}}
\end{aligned}
$$

Note that the result in Theorem 7.6 says the sensitivity operator, $s$, satisfies $M(q) s=-\mathscr{M}(q) u$, i.e.,

$$
\dot{s}(t)+A(q) s(t)=-\mathscr{A}(q) u
$$

which by definition is

$$
\begin{aligned}
\langle\dot{s}(t), \psi\rangle_{\mathcal{V}^{*}, \mathcal{V}}+ & \left\langle\frac{1}{g} k \nabla s(t), \nabla \psi\right\rangle_{\mathcal{H}}+h \int_{\gamma_{2}}\left(\operatorname{Tr}_{2} s(t)\right)(z)\left(\operatorname{Tr}_{2} \psi\right)(z) d S_{2} \\
& =\left\langle-\frac{1}{g} D_{q} k(q) \nabla u(t), \nabla \psi\right\rangle_{\mathcal{H}} .
\end{aligned}
$$

Clearly this is equivalent to (7.9) and so equation (7.9) matches our formal derivation.

Returning to equation (7.9), we see it is equivalent to

$$
\begin{aligned}
\langle\dot{s}(t), \psi\rangle_{\mathcal{V}^{*}, \mathcal{V}}= & \langle-k \nabla s(t), \nabla \psi\rangle_{L^{2}}-h \int_{\gamma_{2}}\left(\operatorname{Tr}_{2} s(t)\right)(z)\left(\operatorname{Tr}_{2} \psi\right)(z) d S_{2} \\
& +\langle-f(t), \nabla \psi\rangle_{L^{2}}
\end{aligned}
$$

by the definition of the $\mathcal{H}$-norm.

Now, if $s \in L^{2}(0, T ; V), k \nabla s \in L^{2}(0, T ; V)$, and $f \in L^{2}(0, T ; V)$, using the Divergence Theorem and the identity

$$
\nabla \cdot(k(z) \nabla s(t, z) \psi(z))=(\nabla \cdot(k(z) \nabla s(t, z)) \psi(z)+k(z)(\nabla s(t, z) \cdot \nabla \psi(z))
$$

in the previous equation, we see

$$
\langle\dot{s}(t), \psi\rangle_{\mathcal{V}^{*}, \mathcal{V}}=\langle\nabla \cdot(k \nabla s(t)), \psi\rangle_{L^{2}}-\int_{\partial \Omega}\left(\operatorname{Tr}_{\Omega}(n \cdot k \nabla s(t))\right)(z)\left(\operatorname{Tr}_{\Omega} \psi\right)(z) d S
$$




$$
\begin{aligned}
& -h \int_{\gamma_{2}}\left(\operatorname{Tr}_{2} s(t)\right)(z)\left(\operatorname{Tr}_{2} \psi\right)(z) d S_{2} \\
& -\int_{\partial \Omega}\left(\operatorname{Tr}_{\Omega}(n \cdot f(t))\right)(z)\left(\operatorname{Tr}_{\Omega} \psi\right)(z) d S+\langle\nabla \cdot f(t), \psi\rangle_{L^{2}}
\end{aligned}
$$

where $\operatorname{Tr}_{\Omega}$ is the continuous trace operator mapping $f \in H^{1}(\Omega) \rightarrow H^{\frac{1}{2}}(\partial \Omega)$ with $\left(\operatorname{Tr}_{\Omega} f\right)(z)=\left.f(z)\right|_{\partial \Omega}$.

If we have enough smoothness on $s$ with respect to the time derivative, i.e., $\dot{s} \in$ $L^{2}(0, T ; \mathcal{H})$, then

$$
\langle\dot{s}(t), \psi\rangle_{\mathcal{V}^{*}, \mathcal{V}}=\langle\dot{s}(t), \psi\rangle_{\mathcal{H}}=\langle g \dot{s}(t), \psi\rangle_{L^{2}}
$$

and so

$$
\begin{gathered}
\langle g \dot{s}(t)-\nabla \cdot(k \nabla u(t))-\nabla \cdot f(t), \psi\rangle_{L^{2}}= \\
-\int_{\partial \Omega}\left(\operatorname{Tr}_{\Omega} n \cdot\left(k \nabla s(t)-\frac{\partial k}{\partial q_{p}} \nabla u(t)\right)\right)(z)\left(\operatorname{Tr}_{\Omega} \psi\right)(z) d S \\
-h \int_{\gamma_{2}}\left(\operatorname{Tr}_{2} s(t)\right)(z)\left(\operatorname{Tr}_{2} \psi\right)(z) d S_{2}
\end{gathered}
$$

for all $\psi \in \mathcal{V}$. We know (7.10) holds for all $\psi \in \mathcal{V}=H^{1}(\Omega)$, thus for all $\psi \in H_{0}^{1}(\Omega)$,

$$
\langle g \dot{s}(t)-\nabla \cdot(k \nabla u(t))-\nabla \cdot f(t), \psi\rangle_{L^{2}}=0 .
$$

Since $H_{0}^{1}(\Omega)$ is a dense subset of $L^{2}(\Omega)$, we know

$$
g \dot{s}(t)-\nabla \cdot(k \nabla u(t))-\nabla \cdot f(t)=0
$$

in the $L^{2}$ sense. Thus we can rewrite (7.10) as

$$
\begin{aligned}
-\int_{\partial \Omega} & \left(\operatorname{Tr}_{\Omega} n \cdot\left(k \nabla s(t)-\frac{\partial k}{\partial q_{p}} \nabla u(t)\right)\right)(z)\left(\operatorname{Tr}_{\Omega} \psi\right)(z) d S \\
- & h \int_{\gamma_{2}}\left(\operatorname{Tr}_{2} s(t)\right)(z)\left(\operatorname{Tr}_{2} \psi\right)(z) d S_{2}=0 .
\end{aligned}
$$

We know $\partial \Omega=\gamma_{1} \cup \gamma_{2} \cup \gamma_{3} \cup \gamma_{4}$. Thus for $\psi \in H_{\gamma_{1}} \equiv\left\{\phi \in H^{1}(\Omega):\left.\phi\right|_{\gamma_{2}, \gamma_{3}, \gamma_{4}}=\right.$ $0\} \subset H^{1}(\Omega)$, by $(7.11)$ we see

$$
-\int_{\gamma_{1}}\left(\operatorname{Tr}_{1} n \cdot\left(k \nabla s(t)+\frac{\partial k}{\partial q_{p}} \nabla u\right)\right)(z)\left(\operatorname{Tr}_{1} \psi\right)(z) d S_{1}=0
$$


and so $\left.k(z) \frac{\partial s}{\partial n}(t, z)\right|_{\gamma_{1}}=-\left.\frac{\partial k}{\partial q_{p}}(z) \frac{\partial u}{\partial n}(t, z)\right|_{\gamma_{1}}$. A similar argument shows $\left.k(z) \frac{\partial s}{\partial n}(t, z)\right|_{\gamma_{3}}=-\left.\frac{\partial k}{\partial q_{p}}(z) \frac{\partial u}{\partial n}(t, z)\right|_{\gamma_{3}}$ and $\left.k(z) \frac{\partial s}{\partial n}(t, z)\right|_{\gamma_{4}}=-\left.\frac{\partial k}{\partial q_{p}}(z) \frac{\partial u}{\partial n}(t, z)\right|_{\gamma_{4}}$.

For $\psi \in H_{\gamma_{2}} \equiv\left\{\phi \in H^{1}(\Omega):\left.\phi\right|_{\gamma_{1}, \gamma_{3}, \gamma_{4}}=0\right\} \subset H^{1}(\Omega)$, using (7.11) we see

$$
\begin{aligned}
-\int_{\gamma_{2}}\left(\operatorname{Tr}_{2} n \cdot\left(k \nabla s(t)+\frac{\partial k}{\partial q_{p}} \nabla u\right)\right) & (z)\left(\operatorname{Tr}_{2} \psi\right)(z) d S_{2} \\
& -h \int_{\gamma_{2}}\left(\operatorname{Tr}_{2} s(t)\right)(z)\left(\operatorname{Tr}_{2} \psi\right)(z) d S_{2}=0
\end{aligned}
$$

and thus $\left.k(z) \frac{\partial s}{\partial n}(t, z)\right|_{\gamma_{2}}=-\left.\left(\frac{\partial k}{\partial q_{p}}(t, z) \frac{\partial u}{\partial n}(t, x)+h s(t, z)\right)\right|_{\gamma_{2}}$. Thus if $s$ satisfies (7.9), and $f$ and $s$ have sufficient additional regularity, $s$ provides a strong solution to (7.6).

Thus (7.9) is the weak or variational form of (7.6), and hence any solution $s$ of (7.9) (if it exists) is a weak solution of (7.6).

\subsubsection{Well-Posedness}

We next show existence of solutions to parabolic systems of the form

$$
\left\{\begin{array}{l}
\dot{s}+\mathcal{A} s=\mathcal{F} \quad \text { in } \mathcal{V}^{*} \\
s(0)=s_{0} .
\end{array}\right.
$$

We use the Gelfand triple $\mathcal{V} \hookrightarrow \mathcal{H} \cong \mathcal{H}^{*} \hookrightarrow \mathcal{V}^{*}$ as in Section 5.2.1. Since $\beta$ defined in (7.7) is equivalent to $\sigma$ defined in (5.8), we know $\beta$ satisfies the following two standard conditions:

(1) The form $\beta$ is $\mathcal{V}$-bounded: for all $\phi, \psi \in \mathcal{V}$, there exists a $\bar{B}<\infty$ such that

$$
|\beta(\phi, \psi)| \leq \bar{B}|\phi|_{\nu}|\psi|_{\nu}
$$

(2) The form $\beta$ is $\mathcal{V}$-coercive: for all $\phi \in \mathcal{V}$, there exists a $\bar{C}<0$ such that

$$
|\beta(\phi, \phi)| \geq \bar{C}|\phi|_{v}^{2}
$$

We also observe that the forcing term $\mathcal{F}$ defined in (7.8) satisfies

$$
\mathcal{F} \in L^{2}\left(0, T ; \mathcal{V}^{*}\right) .
$$


In order to see that (7.15) holds, recall $f \in L^{2}\left(0, T ; L^{2}(\Omega)^{2}\right)$. Thus $\mathcal{F}(t): \mathcal{V} \rightarrow \mathbb{R}$ and is linear, i.e., $\mathcal{F}(t) \in \mathcal{V}^{*}$ and so $\mathcal{F} \in L^{2}\left(0, T ; \mathcal{V}^{*}\right)$.

Given the above hypothesis, the weak or variational form of the system (7.12) is

$$
\left\{\begin{array}{l}
\langle\dot{s}(t), \psi\rangle+\beta(s(t), \psi)=\langle\mathcal{F}(t), \psi\rangle \\
u(0)=u_{0}
\end{array}\right.
$$

for $\psi \in \mathcal{V}$ and $\langle\cdot, \cdot\rangle$ is $\langle\cdot, \cdot\rangle_{\mathcal{V}^{*}, \mathcal{V}}$. Note the system (7.12) is the same as (7.16).

At this point, existence, uniqueness, and continuous dependence on the forcing function follow using an argument analogous to the arguments in Section 5.2. Thus the sensitivity system is well-posed.

\subsubsection{Matlab Solutions}

Using Matlab's PDE Toolbox, we are able to numerically solve the sensitivity equation system. Since we are interested in the temperature of the composite silicone at the heat sink interface, i.e., the $\gamma_{2}$ boundary, we want to know the sensitivity at the heat sink interface as a function of $q_{p}$. Recall that in our model we assume the particles never touch any boundary of the composite silicone. (A reasonable assumption based on the properties of the silicone polymer.) Thus $\left.\frac{\partial k}{\partial q_{p}}(z ; q)\right|_{\gamma_{j}}=0$ for $j=1,2,3,4$ and we can reduce $(7.6)$ to

$$
\left\{\begin{array}{l}
g(z) \dot{s}(t, z ; q)=\nabla \cdot(k(z ; q) \nabla s(t, z ; q))+\nabla \cdot f(t, z ; q) \\
\left.k(z ; q) \frac{\partial s}{\partial n}(t, z ; q)\right|_{\gamma_{4}}=0 \\
\left.k(z ; q) \frac{\partial s}{\partial n}(t, z ; q)\right|_{\gamma_{2}}=-\left.h s(t, z ; q)\right|_{\gamma_{2}} \\
\left.k(z ; q) \frac{\partial s}{\partial n}(t, z ; q)\right|_{\gamma_{1}}=0 \\
\left.k(z ; q) \frac{\partial s}{\partial n}(t, z ; q)\right|_{\gamma_{3}}=0 \\
s(0, z ; q)=0
\end{array}\right.
$$

To solve (7.17), we first fix a value for $q_{p}$ and solve (7.3). Then with that solution we can solve (7.17) with the same fixed value of $q_{p}$. We will define the average sensitivity 
at the boundary $\gamma_{2}$ for a fixed $q_{p}$ at time $t_{i}$ by

$$
s_{2}\left(t_{i} ; q_{p}\right)=\frac{1}{\left|\gamma_{2}\right|} \int_{\gamma_{2}} \operatorname{Tr}_{2} s\left(t_{i} ; q_{p}\right)(z) d S_{2}
$$

where $\operatorname{Tr}_{2}$ is again the continuous trace operator from $H^{1}(\Omega) \rightarrow H^{\frac{1}{2}}\left(\gamma_{2}\right)$ defined by $\left(\operatorname{Tr}_{2} f\right)(z)=\left.f(z)\right|_{\gamma_{2}}$. Repeating this process for different values of $q_{p}$, we can then plot the average sensitivity at the heat sink interface, $s_{2}$, as a function of $q_{p}$. We fix $k_{s}=\bar{q}_{s}=0.12 \mathrm{~W} / \mathrm{mK}$. The remainder of the parameters are given in Table 3.1.

In Figures 7.1 and 7.2 we depict the plots of the average of the solution to (7.17) along the $\gamma_{2}$ boundary, as described in equation (7.18). We let $q_{p}=100,110, \ldots, 1100$ and solve at each of the times $t_{2}, t_{3}, \ldots, t_{8}$ given in Table 3.2. Note that as a function of $q_{p}$, there is variation in the average sensitivity along the heat sink interface at each time step, however the variation is not very substantial when considered with respect to the magnitude of the average solution. To see this, we define the relative average sensitivity to the thermal conductivity of the particles by

$$
s_{2 r}\left(t_{i} ; q_{p}\right)=\frac{s_{2}\left(t_{i} ; q_{p}\right)}{\max _{q_{p} \in Q}\left(s_{2}\left(t_{i} ; q_{p}\right)\right)}
$$

In Figures 7.3 and 7.4 we depict the plots of $s_{2 r}\left(t_{i} ; q_{p}\right)$. Note that these graphs are relatively constant.

In contrast, in Section 7.3.2 we present plots of the average sensitivity with respect to the thermal conductivity of the silicone and of the average relative sensitivity with respect to the thermal conductivity of the silicone. In all of the plots there is a substantial variation in the sensitivity as a function of $q_{s}$. Thus, based on our results in this section and in Section 7.3.2, we conclude the solution $u$ to (7.3) is not very sensitive to the thermal conductivity of the particles. 

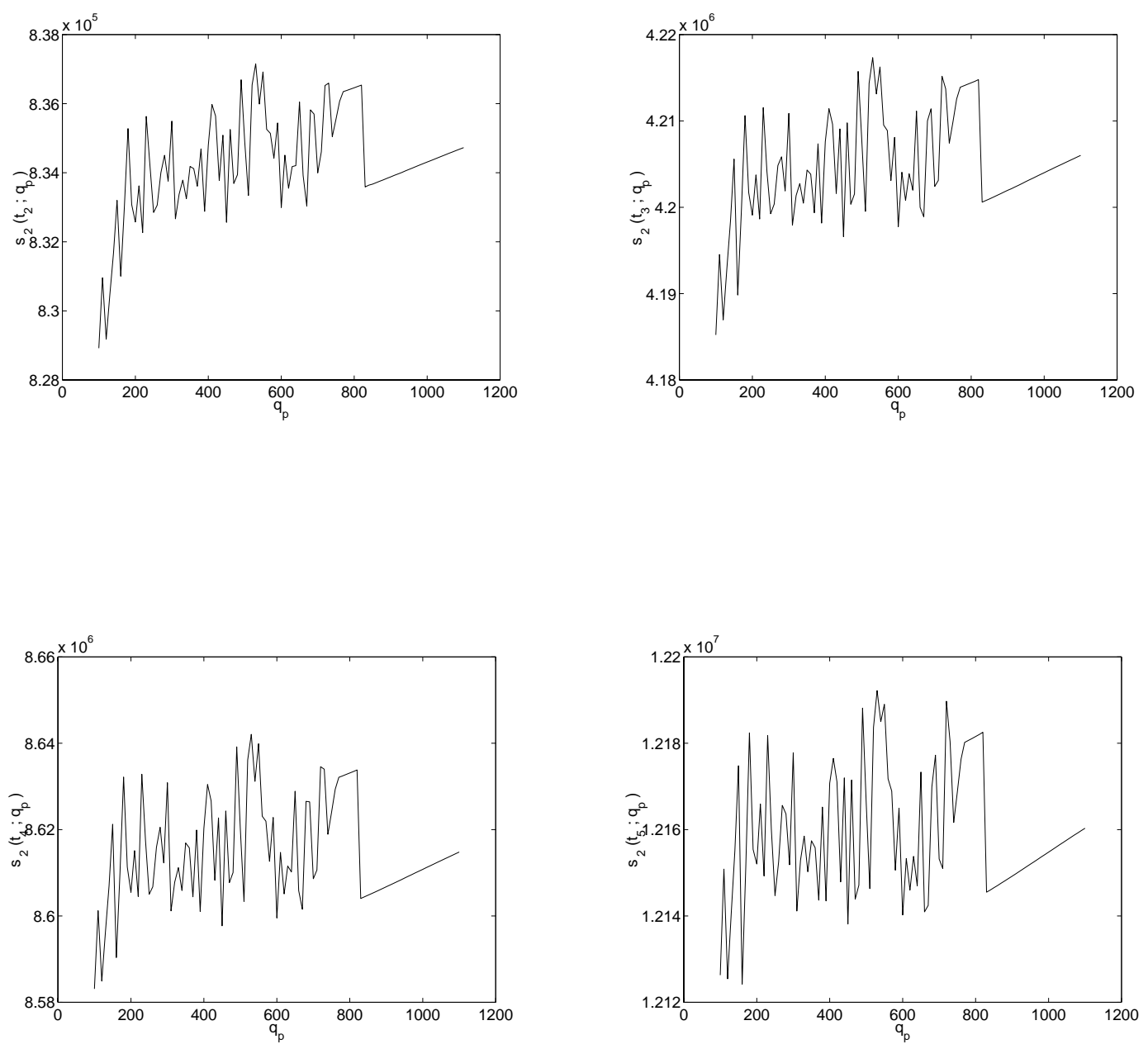

Figure 7.1: Average sensitivity at $\gamma_{2}$ at times $t_{2}, t_{3}, t_{4}$, and $t_{5}$ as a function of $q_{p}$ 

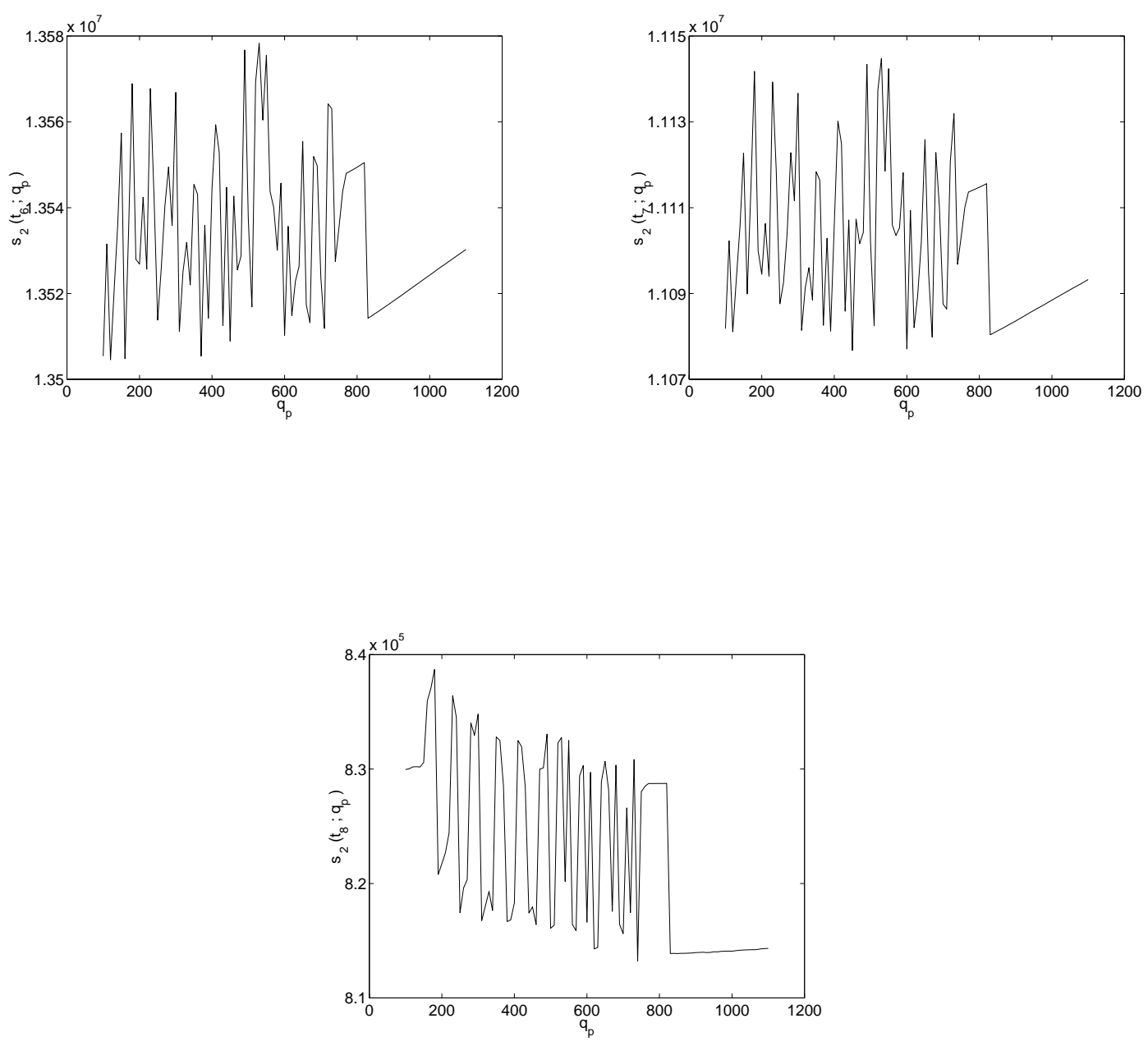

Figure 7.2: Average sensitivity at $\gamma_{2}$ at times $t_{6}, t_{7}$, and $t_{8}$ as a function of $q_{p}$ 

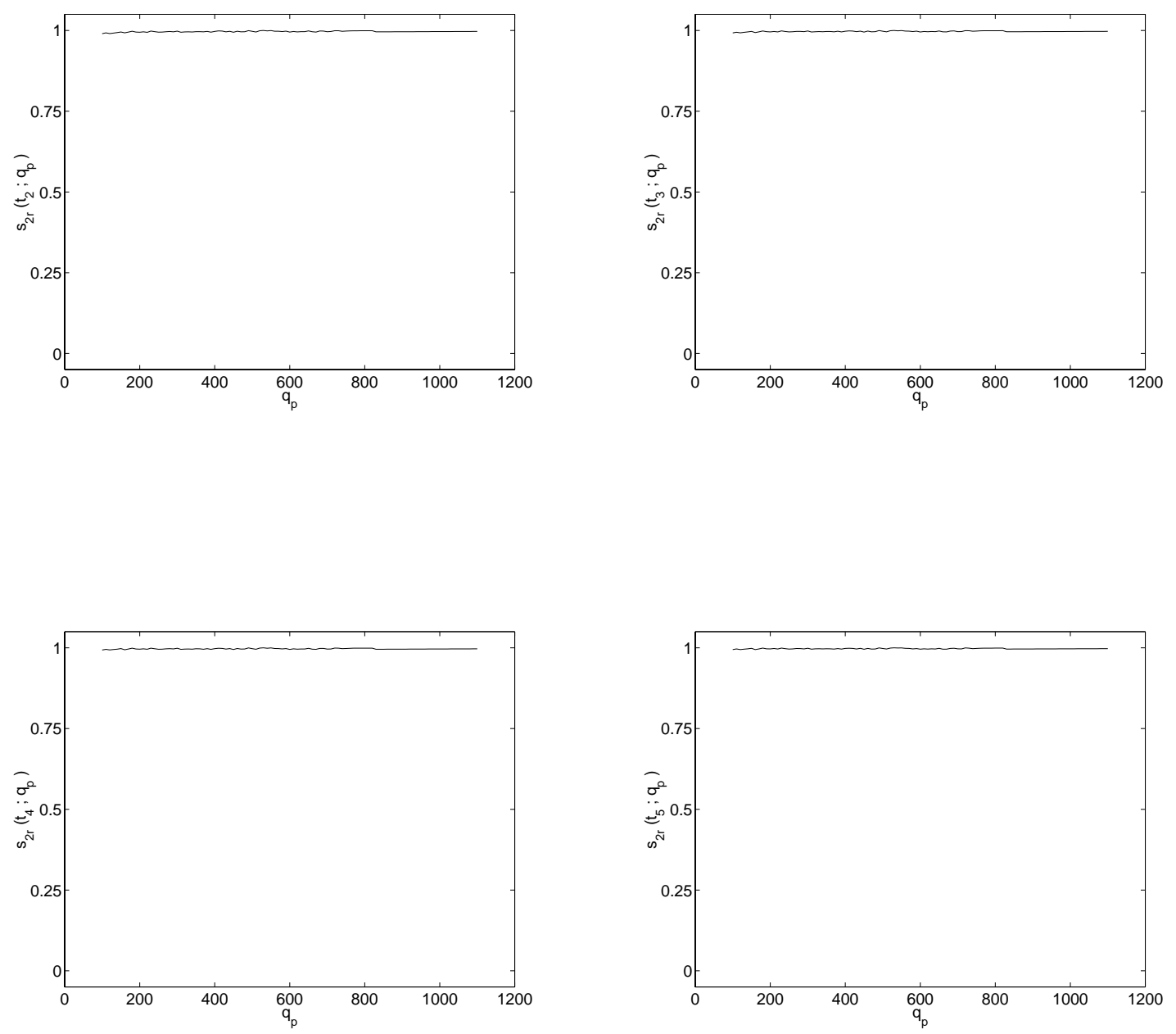

Figure 7.3: Relative average sensitivity at $\gamma_{2}$ at times $t_{2}, t_{3}, t_{4}$, and $t_{5}$ as a function of $q_{p}$ 

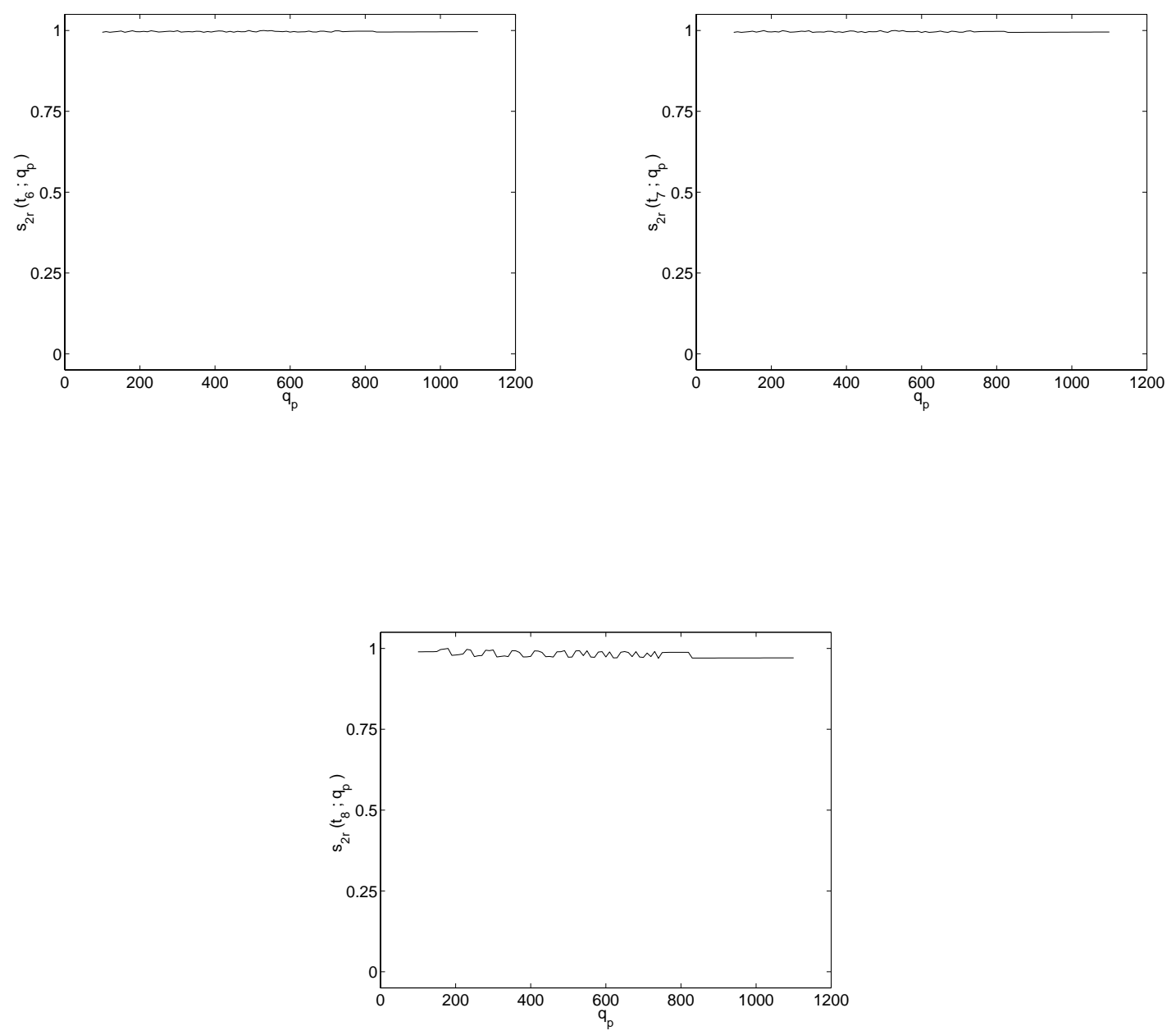

Figure 7.4: Relative average sensitivity at $\gamma_{2}$ at times $t_{6}, t_{7}$, and $t_{8}$ as a function of $q_{p}$ 


\subsection{Sensitivity to the Silicone Polymer Thermal Conductivity}

\subsubsection{Derivation of Sensitivity Equations}

We again begin with (7.3), and again we assume the thermal conductivity $k(z)$ is given by

$$
k(z ; q)= \begin{cases}q_{s} & z \in \Omega_{s} \\ \bar{q}_{p} & z \in \Omega_{p}\end{cases}
$$

where $\bar{q}_{p}$ is a constant, and $q_{s}$ is varied in a range of admissible values. Any weak solution of $(7.3)$ will again have the form $u(t, z ; q)$. In order to derive the sensitivity equations we will differentiate (7.3) with respect to $q_{s}$.

The differentiation follows in a manner similar to the differentiation in Section 7.2.1. We will define the sensitivity to $q_{s}$ as $w(t, z ; q)=\frac{\partial u}{\partial q_{s}}(t, z ; q)$. We will assume the source flux, $S_{0}(t)$ and the initial condition, $\Upsilon(z)$ are independent of $q_{s}$.

It is important to note that

$$
\frac{\partial k}{\partial q_{s}}(z ; q)=\left\{\begin{array}{cc}
1 & z \in \Omega_{s} \\
0 & z \in \Omega_{p}
\end{array}\right.
$$

and so $\frac{\partial k}{\partial q_{s}}(z ; q) \in L^{\infty}(\Omega)$. Furthermore, $\frac{\partial k}{\partial q_{p}}(z ; q)=0$ since we are holding $q_{p}=\bar{q}_{p}$ fixed. Thus, since $q=\left(q_{p}, q_{s}\right), D_{q} k(q)=\left(\frac{\partial k}{\partial q_{p}}, \frac{\partial k}{\partial q_{s}}\right)=\left(0, \frac{\partial k}{\partial q_{s}}\right)$. Recall $u \in L^{2}(0, T ; \mathcal{V})$, and so $\nabla u \in L^{2}\left(0, T ; L^{2}(\Omega)^{2}\right)$. Then if we define

$$
f_{s}(t, z ; q)=\frac{\partial k}{\partial q_{s}}(z ; q) \nabla u(t, z ; q)
$$

we have $f_{s}(\cdot, \cdot ; q) \in L^{2}\left(0, T ; L^{2}(\Omega)^{2}\right)$. 
Thus we formally have the following system for our sensitivity equation:

$$
\left\{\begin{array}{l}
g(z) \dot{w}(t, z ; q)=\nabla \cdot(k(z ; q) \nabla w(t, z ; q))+\nabla \cdot f_{s}(t, z ; q) \\
\left.k(z ; q) \frac{\partial w}{\partial n}(t, z ; q)\right|_{\gamma_{4}}=-\left.\frac{\partial k}{\partial q_{s}}(z ; q) \frac{\partial u}{\partial n}(t, z ; q)\right|_{\gamma_{4}} \\
\left.k(z ; q) \frac{\partial w}{\partial n}(t, z ; q)\right|_{\gamma_{2}}=-\left.\left(\frac{\partial k}{\partial q_{s}}(z ; q) \frac{\partial u}{\partial n}(t, z ; q)+h w(t, z ; q)\right)\right|_{\gamma_{2}} \\
\left.k(z ; q) \frac{\partial w}{\partial n}(t, z ; q)\right|_{\gamma_{1}}=-\left.\frac{\partial k}{\partial q_{s}}(z ; q) \frac{\partial u}{\partial n}(t, z ; q)\right|_{\gamma_{1}} \\
\left.k(z ; q) \frac{\partial w}{\partial n}(t, z ; q)\right|_{\gamma_{3}}=-\left.\frac{\partial k}{\partial q_{s}}(z ; q) \frac{\partial u}{\partial n}(t, z ; q)\right|_{\gamma_{3}} \\
w(0, z ; q)=0
\end{array}\right.
$$

Using an argument analogous to the arguments in Section 7.2.2 and Section 7.2.3 it can be shown there exists a unique weak solution $w$ to (7.19) and that the problem is well-posed.

\subsubsection{Matlab Solutions}

As in Section 7.2.4 we can use Matlab to solve the sensitivity equation (7.19) for different values of $q_{s}$. Since we assume the particles never touch any boundary of the composite silicone, $\left.\frac{\partial k}{\partial q_{s}}\right|_{\gamma_{j}}=1$ for $j=1,2,3,4$ and so we can reduce (7.19) to

$$
\left\{\begin{array}{l}
g(z) \dot{w}(t, z ; q)=\nabla \cdot(k(z ; q) \nabla w(t, z ; q))+\nabla \cdot f_{s}(t, z ; q) \\
\left.k(z ; q) \frac{\partial w}{\partial n}(t, z ; q)\right|_{\gamma_{4}}=-S_{0}(t) \\
\left.k(z ; q) \frac{\partial w}{\partial n}(t, z ; q)\right|_{\gamma_{2}}=-\left.\left(h\left(T_{\infty}-u(t, z ; q)\right)+h w(t, z ; q)\right)\right|_{\gamma_{2}} \\
\left.k(z ; q) \frac{\partial w}{\partial n}(t, z ; q)\right|_{\gamma_{1}}=0 \\
\left.k(z ; q) \frac{\partial w}{\partial n}(t, z ; q)\right|_{\gamma_{3}}=0 \\
w(0, z ; q)=0
\end{array}\right.
$$

We fix $k_{p}=\bar{q}_{p}=217 \mathrm{~W} / \mathrm{mK}$. The remaining parameters are given in Table 3.1. In order to implement the boundary condition for $\gamma_{2}$ in the PDE Toolbox, we will use the average value of $u(t, z ; q)$ on $\gamma_{2}$ for $u(t, z ; q)$ in $\left.k(z ; q) \frac{\partial w}{\partial n}(t, z ; q)\right|_{\gamma_{2}}=-\left(h\left(T_{\infty}-\right.\right.$ $u(t, z ; q))+h w(t, z ; q))\left.\right|_{\gamma_{2}}$. 
As in Section 7.2.4, we define the average sensitivity at the boundary $\gamma_{2}$ for a fixed $q_{s}$ at time $t_{i}$ by

$$
w_{2}\left(t_{i} ; q_{s}\right)=\frac{1}{\left|\gamma_{2}\right|} \int_{\gamma_{2}} \operatorname{Tr}_{2} w\left(t_{i} ; q_{s}\right)(z) d S_{2}
$$

where $w\left(t_{i} ; q_{s}\right)$ is the solution to $(7.20)$ at $t_{i}$ for a given $q_{s}$ and fixed $q_{p}$. Repeating this process for different values of $q_{p}$, we can then plot the average sensitivity at the heat sink interface, $w_{2}$, as a function of $q_{s}$. .

In Figures 7.5 and 7.6 we present plots of the average solution to (7.20) at $\gamma_{2}$ as a function of $q_{s}$, as defined in equation (7.21). We solved (7.20) with $q_{s}=$ $0.02,0.02, \ldots, 1.02$ at each of the times $t_{2}, t_{3}, \ldots, t_{8}$ given in Table 3.2. In each these graphs it clear that as a function of $q_{s}$ there is significant variation along the heat sink interface. As in the Section 7.2.4, we define the relative average sensitivity to the thermal conductivity of the silicone by

$$
w_{2 r}\left(t_{i} ; q_{s}\right)=\frac{w_{2}\left(t_{i} ; q_{s}\right)}{\max _{q_{s} \in Q}\left(w_{2}\left(t_{i} ; q_{s}\right)\right)} .
$$

In Figures 7.7 and 7.8 we depict the plots of $w_{2 r}\left(t_{i} ; q_{s}\right)$. Note that these graphs still show variation.

Thus we conclude the solution $u$ to (7.3) exhibits significant sensitivity to changes in the thermal conductivity of the silicone. Also, we note the significant difference between the graphs presented here and those in Section 7.2.4.

Based on these results, increasing the thermal conductivity of the particles is not the best approach for improving the overall thermal conductivity of the composite. Clearly the composite is more sensitive to the thermal conductivity of the base material. Thus a better means of improving the overall thermal conductivity of the composite is to increase the thermal conductivity of the base polymer used in the composite. 

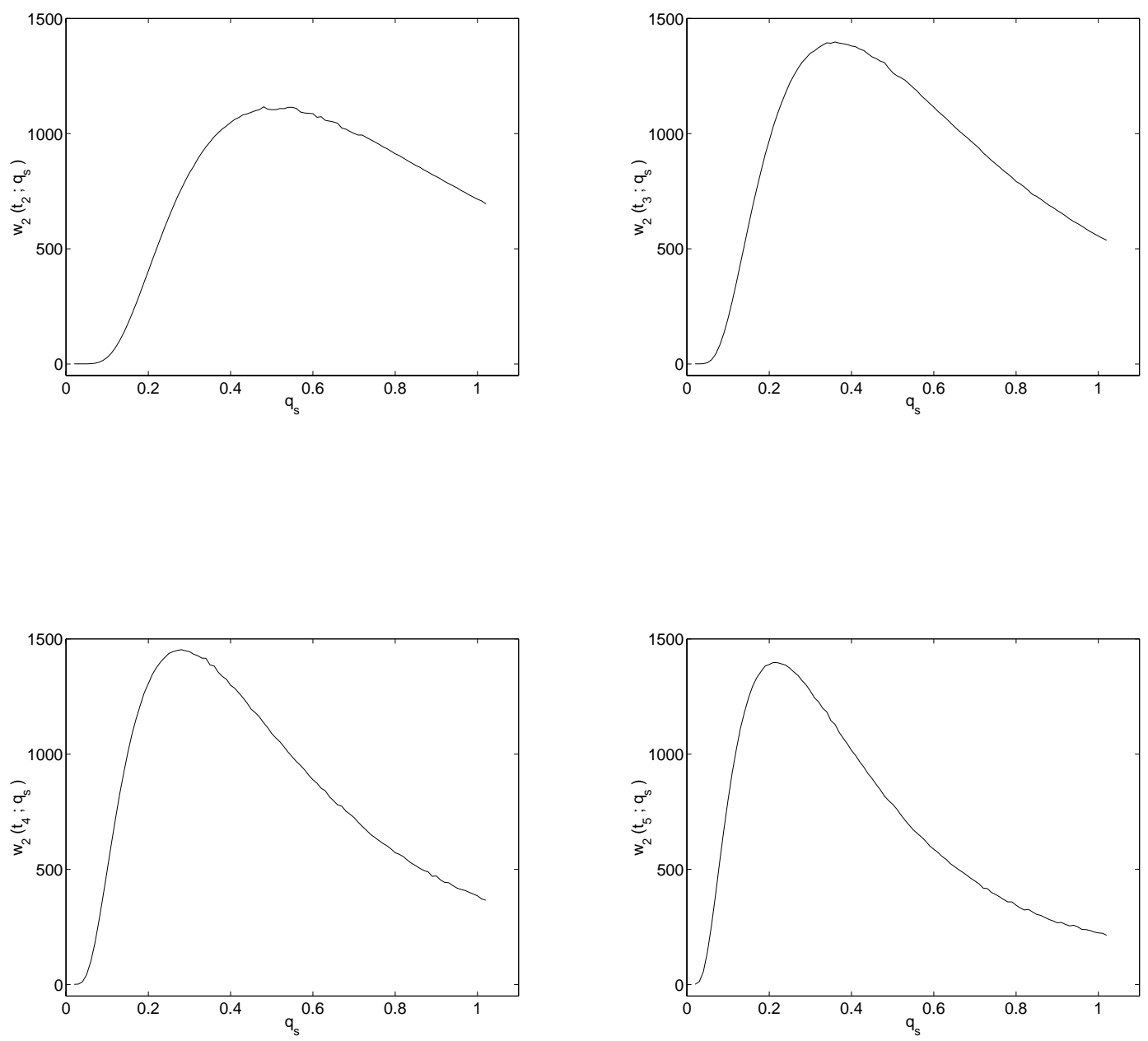

Figure 7.5: Average sensitivity at $\gamma_{2}$ at times $t_{2}, t_{3}, t_{4}$, and $t_{5}$ as a function of $q_{s}$ 

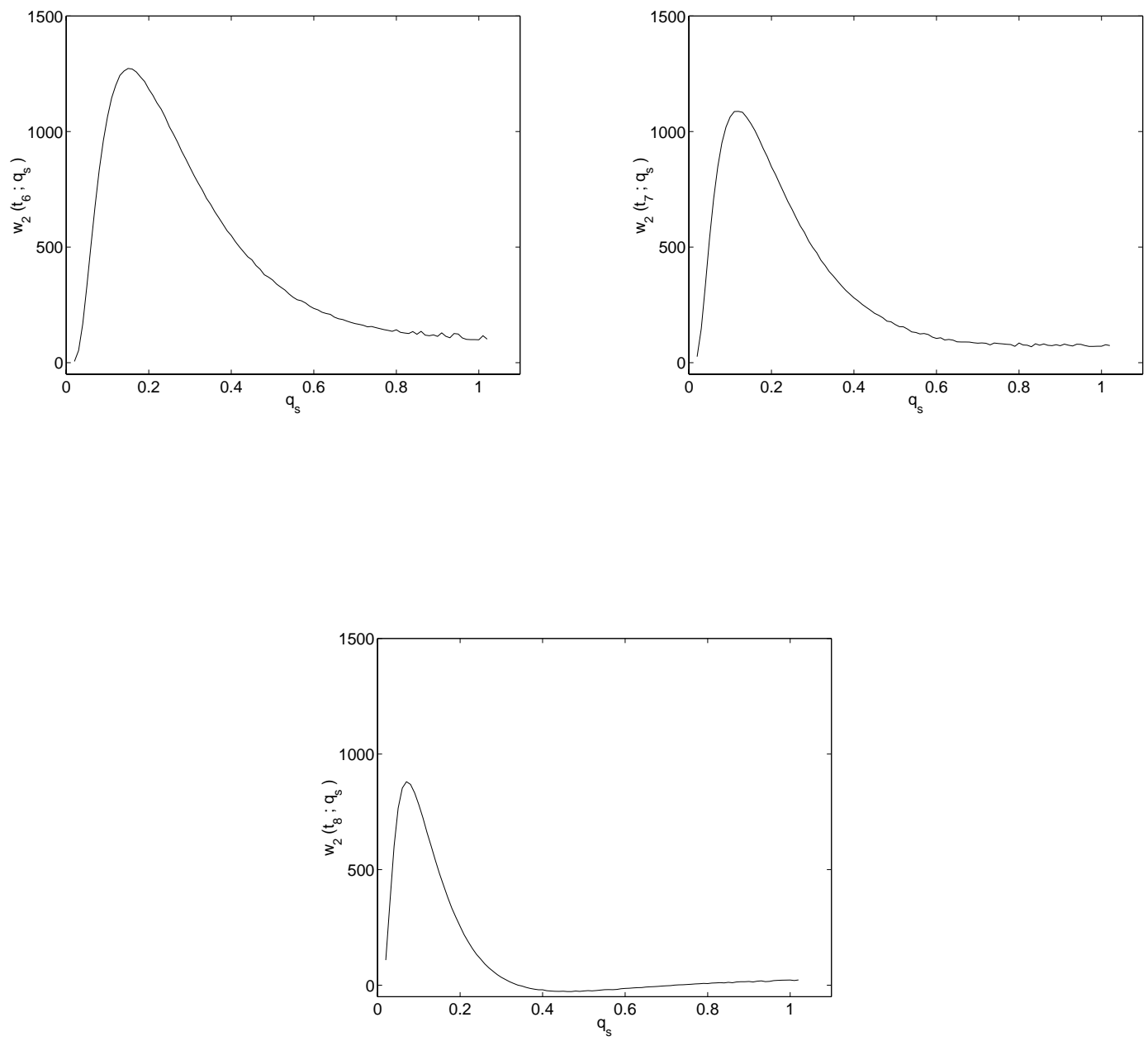

Figure 7.6: Average sensitivity at $\gamma_{2}$ at times $t_{6}, t_{7}$, and $t_{8}$ as a function of $q_{s}$ 

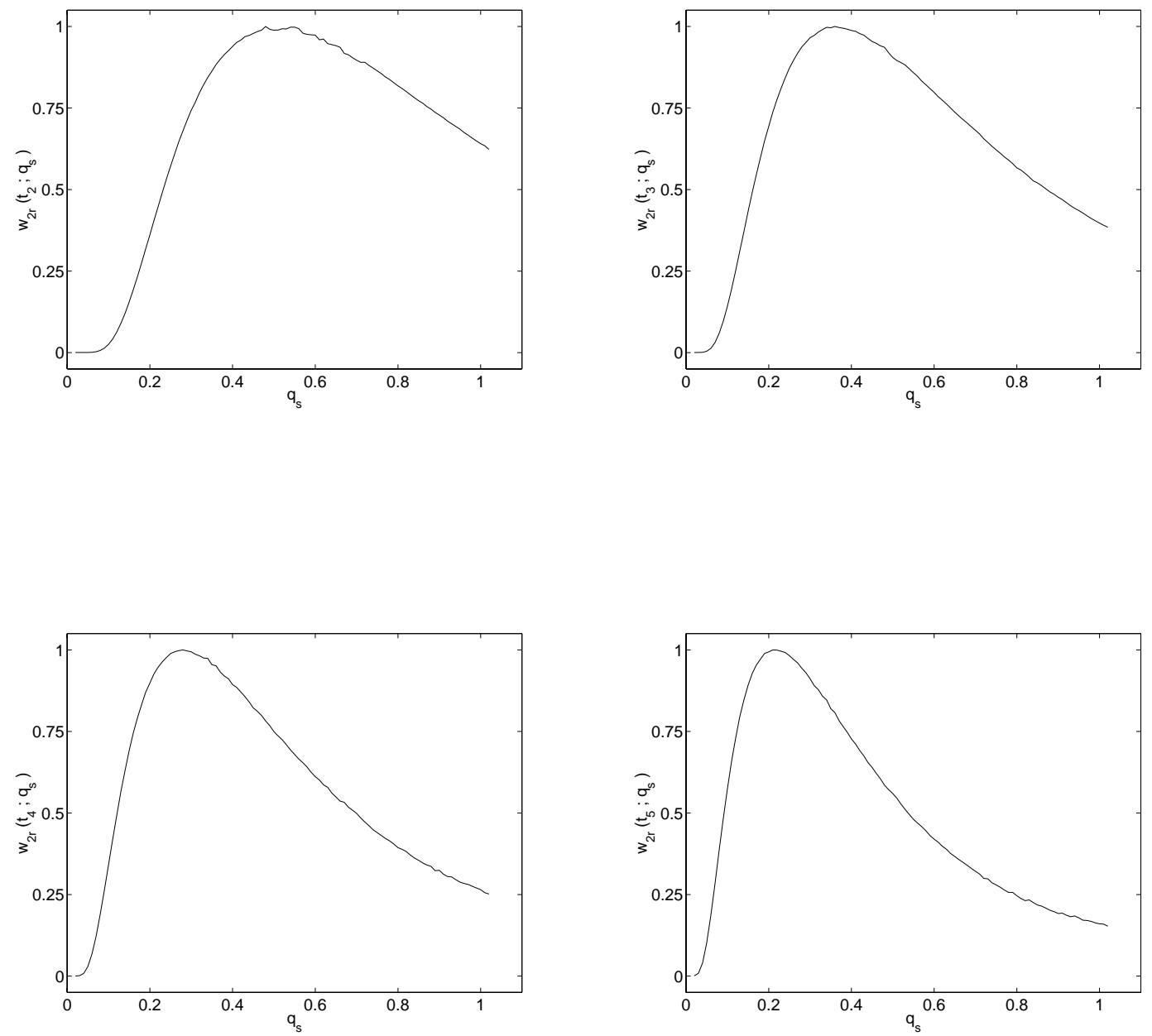

Figure 7.7: Relative average sensitivity at $\gamma_{2}$ at times $t_{2}, t_{3}, t_{4}$, and $t_{5}$ as a function of $q_{s}$ 

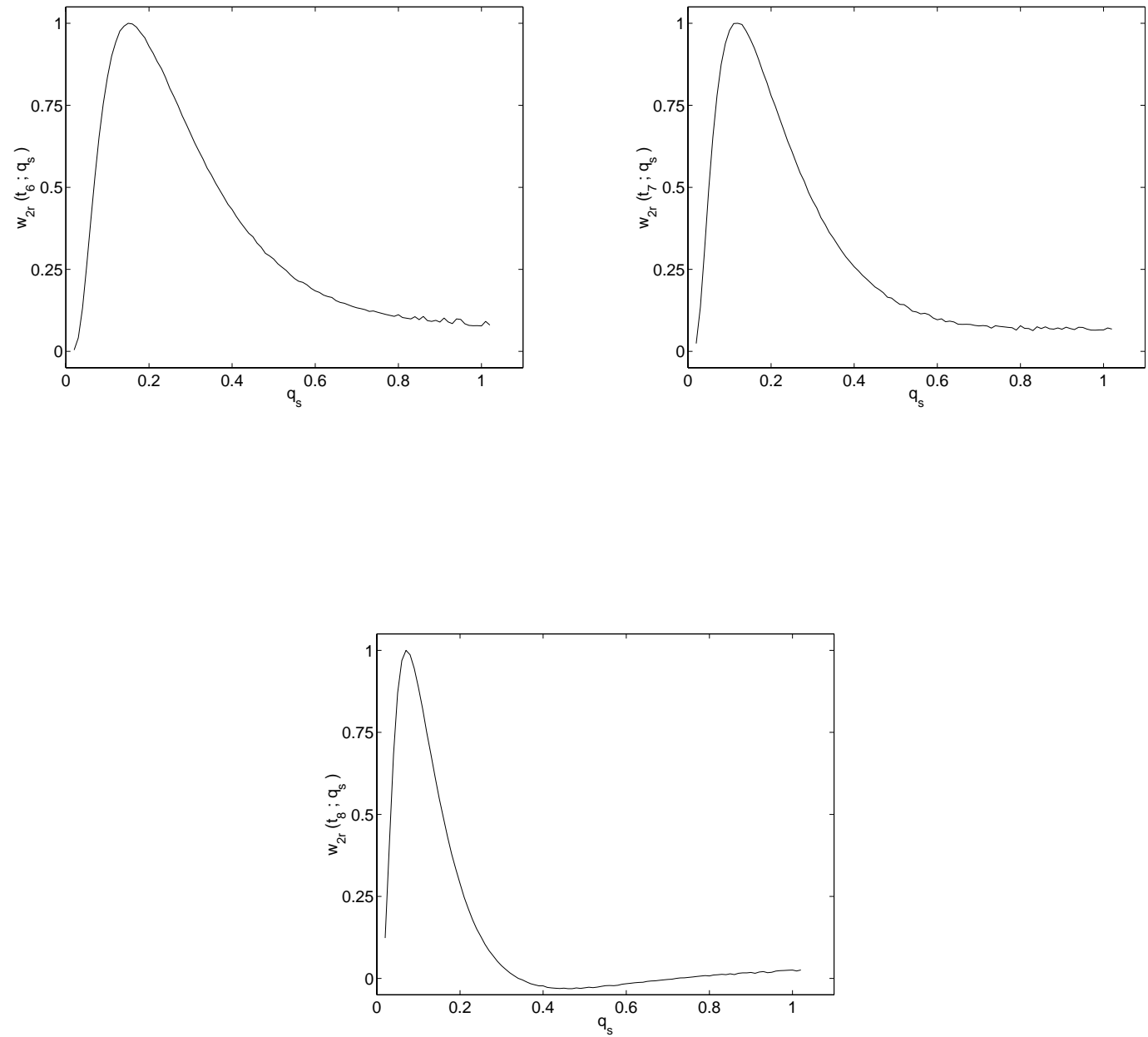

Figure 7.8: Relative average sensitivity at $\gamma_{2}$ at times $t_{6}, t_{7}$, and $t_{8}$ as a function of $q_{s}$ 


\section{Chapter 8}

\section{Conclusions and Future Work}

\subsection{Conclusions}

We have presented a three dimensional model based on the composite silicone and the data collection process. For proof of concept, we reduced the three dimensional model to a two dimensional model. Using Matlab's PDE Toolbox, we presented numerical solutions to our two dimensional model. Matlab's PDE Toolbox allowed us to accommodate the oscillatory coefficients in our computations along with varying particle geometries. We have shown that the geometry of the composite silicone has a significant impact on the heat flux at the interface between the heat sink and the composite. However, based on our these results, we chose to use a uniform geometry for our analysis.

In Chapter 4 we presented the mathematical theory of homogenization. Homogenization combines the oscillatory coefficients into an "average" or "effective" thermal conductivity. While we chose to use the PDE Toolbox for our analysis, we presented homogenization as a viable alternative.

We have proven existence and uniqueness theorems based on our two dimensional model, and have shown the model depends continuously on parameters, as well as the initial data and forcing function. We have presented a formulation and numerical results for two different parameter estimation problems: estimating parameters as 
constants and estimating parameters as realizations of random variables. Estimating parameters as realizations of random variables used a probability based approach, and we have provided the theoretical framework for this approach. In each of our parameter estimation formulations (using simulated data for proof of concept) we were able to accurately estimate the thermal conductivity of the silicone, but not the thermal conductivity of the aluminum filler particles.

After formally deriving the sensitivity equations, we studied the sensitivity of the solution to our two dimensional model to both the thermal conductivity of the particles and the thermal conductivity of the silicone. Our numerical results clearly indicated the solution is significantly more sensitive to the thermal conductivity of the silicone than to the thermal conductivity of the particles. This supported our results from the parameter estimation. Thus, in order to significantly increase the thermal conductivity of the composite silicone (or any composite adhesive), we believe it is best to work at increasing the thermal conductivity of the base silicone (or base adhesive). However, we believe there is still a great deal to learn about thermally conductive adhesives.

\subsection{Future Work}

In Chapter 7, we concluded that the solution to our two dimensional model is significantly more sensitive to changes in the thermal conductivity of the silicone than to changes in the thermal conductivity of the particles. However, our study of the different composite geometries in Section 3.4 indicated that the arrangement of the particles had a noticeable effect on the heat flux at the interface between the heat sink and the composite silicone. It would be interesting to use the sensitivity methods of Chapter 7 to quantify the influence of the particle geometries on the solution. In particular, it would be interesting to see how multiple sized particles, various particle alignments and even different particle shapes impact the heat flow. This is a challenging problem since the boundaries will change as the particle size, alignment and 
shape are changed. However, based on the results in [25, 26, 27, 28] where sensitivity analysis is successfully used on parameter dependent domains, we believe this analysis is possible.

In Chapter 3 we briefly discussed the three dimensional model before reducing to a two dimensional model for proof of concept. Since we are ultimately trying to gain knowledge about three dimensional composite materials, a full development and analysis of a three dimensional model would be beneficial. However, Matlab's Partial Differential Equation Toolbox is not capable of solving three dimensional problems. Thus in order to numerically solve a three dimensional model it is necessary to use a different computational package. Homogenization theory may also be useful for the three dimensional model. However, since it is most likely that the actual composite geometry is random, not periodic, it would be best to consider homogenization theory for random media. This is more challenging than homogenization for periodic media, but there is a significant amount of literature in this field, including $[8,30]$.

Another interesting question is the relationship between the voltage rise and the temperature rise discussed in Section 2.3. In order to accurately use the experimental data with our model, the exact relationship between the voltage and temperature rises needs to understood. This can either be done experimentally with a thermocouple, or with an inverse problem where one of the parameters to be identified is the constant of proportionality. 


\section{List of References}

[1] H. T. BANKS AND K. L. BIHARI, Modeling and estimating uncertainty in parameter estimation, Inverse Problems, 17 (2001), pp. 95-111.

[2] H. T. Banks, D. Cioranescu, And D. A. Rebnord, Homogenization models for two-dimensional grid structures, Asymptotic Analysis, 11 (1995), pp. 107130.

[3] H. T. Banks, D. S. Gilliam, And V. I. Shubov, Global solvability for damped abstract nonlinear hyperbolic systems, Differential and Integral Equations, 10 (1997), pp. 309-332.

[4] H. T. Banks And G. M. Groome, JR., Convergence theorems for parameter estimation by quasilinearization, Journal of Mathematical Analysis and Applications, 42 (1973), pp. 91-109.

[5] H. T. Banks And K. Kunisch, Estimation Techniques for Distributed Parameter Systems, Birkhäuser, Boston, 1989.

[6] H. T. Banks And C. J. Musante, Well-posedness for a class of abstract nonlinear parabolic systems with time delay, Nonlinear Analysis - Theory, Methods \& Applications, 35 (1999), pp. 629-648.

[7] H. T. Banks, R. C. Smith, And Y. Wang, Smart Material Structures: Modeling, Estimation and Control, John Wiley \& Sons, Chichester, 1996. 
[8] A. Bensoussan, J.-L. Lions, and G. Papanicolaou, Asymptotic Analysis for Periodic Structures, North Holland, Amsterdam, 1978.

[9] M. S. Berger, Non Linearity and Functional Analysis : Lectures on Nonlinear Problems in Mathematical Analysis, Pure and Applied Mathematics, Academic Press, New York, 1977.

[10] P. Billingsley, Convergence of Probability Measures, John Wiley \& Sons, New York, 1968.

[11] L. M. Clark And R. E. TAYlor, Radiation loss in the flash method for thermal diffusivity, Journal of Applied Physics, 46 (1975), pp. 714-719.

[12] M. Davidian And D. M. Giltinan, Nonlinear Models for Repeated Measurement Data, Chapman and Hall/CRC, Boca Raton, FL, 1998.

[13] A. Defranceschi, An introduction to homogenization and g-convergence, Lecture Notes, School on Homogenization, ICTP, Trieste, Sept. 6-8 1993.

[14] S. N. Ethier And T. G. Kurtz, Markov Processes Characterization and Convergence, John Wiley \& Sons, New York, 1986.

[15] Holometrix, Inc., Operation Manual - Holometrix Microflash, Bedford, MA, 1997.

[16] F. P. Incropera And D. P. DeWitt, Fundamentals of Heat and Mass Transfer, John Wiley \& Sons, New York, 1990.

[17] J. L. Kelley, General Topology, D. Van Nostrand Co., Princeton, 1955.

[18] B. G. Lindsay, Mixture Models: Theory, Geometry and Applications, vol. 5 of NSF-CBMS Regional Conference Series in Probability and Statistics, Inst. Math. Stat., Hayward, CA, 1995. 
[19] J. L. Lions, Optimal Control of Systems Governed by Partial Differential Equations, Springer-Verlag, New York, 1971.

[20] The Mathworks, Inc., Partial Differential Equation Toolbox User's Guide, 1997.

[21] — Optimization Toolbox User's Guide, 2001.

[22] V. G. Maz'JA, Sobolev Spaces, Springer-Verlag, Berlin, 1985.

[23] T. M. Simon, F. Reitich, M. R. Jolly, K. Ito, and H. T. Banks, Estimation of the effective permeability in magnetorheological fluids, CRSC Technical Report CRSC-TR98-35, N. C. State University, October 1998; Journal of Intelligent Materials Systems and Structures, 10 (1999), pp. 872-879.

[24] — On the effective magnetic properties of magnetorheological fluids, CRSC Technical Report CRSC-TR98-42, N. C. State University, November 1998; Mathematical and Computer Modeling, 33 (2001), pp. 273-284.

[25] L. G. Stanley, Computational Methods for Sensitivity Analysis with Applications to Elliptic Boundary Value Problems, PhD thesis, Virginia Polytechnic Institute and State University, Blacksburg, Virginia, July 1999.

[26] — A sensitivity equation method for molding processes, in Proceedings of the IEEE CCA/CACSD, September 2000.

[27] _ - Shape sensitivities for optimal design: A case study on the use of continuous sensitivity equation methods, in Nonsmooth/Nonconvex Mechanics : Modeling, Analysis, and Numerical Methods, D. Y. Gao, R. W. Ogden, and G. E. Stavroulakis, eds., vol. 50 of Nonconvex Optimization and Its Application, Kluwer Academic Publishers, Dordrecht, March 2001, pp. 209-229.

[28] — Sensitivity equation methods for parameter dependent elliptic equations, Numerical Functional Analysis and Optimization, (to appear). 
[29] J. WlokA, Partial Differential Equations, Cambridge University Press, Cambridge, 1987.

[30] V. V. Zhikov, S. M. Kozlov, and O. A. Oleinik, Homogenization of Differential Operators and Integral Functionals, Springer-Verlag, New York, 1994. 